\title{
Development of the 1,2-oxaza-Cope Rearrangement
}

Armen Zakarian* and Chong-Dao Lu

\section{Supporting Information}

Experimental procedures, spectroscopic and analytical data for compounds $1 \mathrm{c}, 1 \mathrm{f}, 3,3 \mathrm{a}-3 \mathrm{~g}$.

General Information. All reactions were carried out under an inert atmosphere of dry argon in oven or flame-dried glassware. Proton magnetic resonance spectra were recorded at 300 and $500 \mathrm{MHz}$ on Varian Mercury and Varian Unity Inova spectrometers, respectively. Carbon magnetic resonance spectra were recorded at $75 \mathrm{MHz}$ on a Varian Mercury spectrometer. All chemical shifts were reported in $\delta$ units relative to tetramethylsilane. Infrared spectra were recorded on Perkin-Elmer Paragon 1000 FTIR spectrometer. High resolution mass spectral data were obtained by the Mass spectrometry Laboratory at the Florida State University. Analytical thin-layer chromatography (TLC) was performed using pre-coated TLC plates with silica Gel 60 F254 (E. Merck no. 57157). Flash column chromatography was performed using 40-63 um silica gel (Merck, Geduran, no. 11567-1) as the stationary phase. Tetrahydrofuran (THF) was dried by distilling from sodium-benzophenone in a continuous still under an atmosphere of argon. Dichloromethane, di-iso-propylamine, triethylamine, and chlorotrimethylsilane were distilled from calcium hydride in a continuous still under an atmosphere of argon. All reagents were used as purchased unless noted otherwise.

Compounds $1,{ }^{1} \mathbf{1 a},{ }^{2,4} \mathbf{1 b},{ }^{3} \mathbf{1 d},{ }^{4} \mathbf{1 e},{ }^{5}$ and $1 \mathbf{g}^{6}$ have been prepared according to the literature procedures.

\footnotetext{
${ }^{1}$ (a) Maskill, H.; Wilson, A. A. J. Chem. Soc. Perkin Trans 2 1984, 119. (b) Inukai, T.; Kasai, M. J. Org. Chem. 1965, 30 , 3567. (c) Mathieu, B.; Ghosez, L. Tetrahedron 2002, 58, 8219.

${ }^{2}$ Sudo, A.; Saigo, K. Chem. Lett. 1997, 97.

${ }_{3}^{3}$ Mirrington, R. N.; Schmalzl, K. J. J. Org. Chem. 1969, 34, 2358.

${ }^{4}$ Ikota, N. Chem. Pharm. Bull. 1989, 37, 2219.

${ }^{5}$ Orita, A.; Nagano, Y.; Hirano, J.; Otera, J.; Syn. Lett. 2001, 637.

${ }^{6}$ Minger, T. L.; Phillips, A. J. Tetrahedron Lett. 2002, 43, 5357.
} 


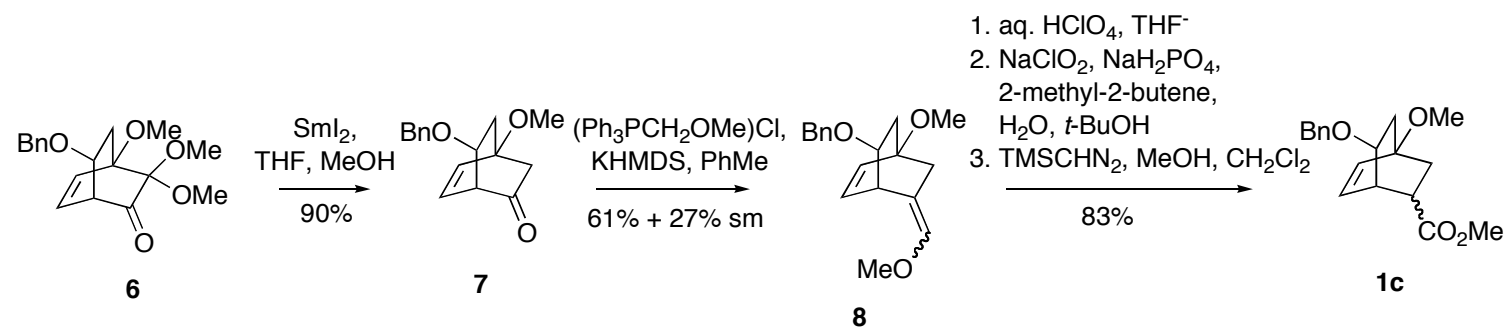

Esters 1c. To a solution of $\alpha, \alpha$-dimethoxy-ketone $6^{7}(610 \mathrm{mg}, 1.92$ mmol) in methanol ( $1 \mathrm{~mL}$ ) was added $\mathrm{SmI}_{2}$ solution (0.1M in THF) (80 $\mathrm{mL}, 8$ mmol) at room temperature until the blue color of the reaction mixture did not faded. The mixture was stirred for $10 \mathrm{~min}$ and then diluted with ethyl acetate, washed with solution of $\mathrm{K}_{2} \mathrm{CO}_{3}$. The organic layer was separated and the aqueous layer was extracted three times with ethyl acetate. The combined organic layers was washed with brine, dried over anhydrous $\mathrm{Na}_{2} \mathrm{SO}_{4}$, filtered and concentrated. Silica gel chromatography (30\% ethyl acetate-hexanes) afforded reduction product 7 (446 mg, 1.73 mmol, 90\% yield). ${ }^{1} \mathrm{H} \operatorname{NMR}\left(300 \mathrm{MHz}, \mathrm{CDCl}_{3}\right) ; \delta(\mathrm{ppm}): 7.38-7.27(\mathrm{~m}, 5 \mathrm{H})$; $6.66(\mathrm{~d}, \mathrm{~J}=9.0 \mathrm{~Hz}, 1 \mathrm{H}) ; 6.07$ ( dd, J1=9.0 Hz, J2=6.0 Hz, $1 \mathrm{H}) ; 4.52$ (d, $\mathrm{J}=12.0 \mathrm{~Hz}, 1 \mathrm{H}) ; 4.47(\mathrm{~d}, \mathrm{~J}=12.0 \mathrm{~Hz}, 1 \mathrm{H}) ; 4.05-4.00$ (m, 1H); 3.55 (ddd, $\mathrm{J} 1=6.0 \mathrm{~Hz}, \mathrm{~J} 2=3.0 \mathrm{~Hz}, \mathrm{~J} 3=1.2 \mathrm{~Hz}, 1 \mathrm{H}) ; 3.42(\mathrm{~s}, 3 \mathrm{H}) ; 2.30$ ( dd, J1=17.4 $\mathrm{Hz}, \mathrm{J} 2=3.6 \mathrm{~Hz}, 1 \mathrm{H}) ; 2.21(\mathrm{dd}, \mathrm{J} 1=12.6 \mathrm{~Hz}, \mathrm{~J} 2=9.0 \mathrm{~Hz}, 1 \mathrm{H}) ; 2.05$ (d, $\mathrm{J}=17.4 \mathrm{~Hz}, 1 \mathrm{H}) ; 1.81$ (dt, Jd=12.6 Hz, Jt=3.6 Hz, 1H). HRMS (ESI) calcd for $\mathrm{C}_{16} \mathrm{H}_{18} \mathrm{O}_{3} \mathrm{Na}[\mathrm{M}+\mathrm{Na}] 281.1154$, found 281.1154

To a solution of (methoxymethyl)-triphenylphosphonium chloride $(2.1 \mathrm{~g}, 6.10 \mathrm{mmol})$ in $10 \mathrm{ml}$ dry toluene was added solution of KHMDS $(0.5 \mathrm{M}$ in toluene, $11.1 \mathrm{ml}, 5.58 \mathrm{mmol})$ at $0^{\circ} \mathrm{C}$, and the mixture was stirred at $0^{\circ} \mathrm{C}$ for $30 \mathrm{~min}$. Ketone $7(410 \mathrm{mg}, 1.59 \mathrm{mmol})$ in toluene (5 ml) was then added and the reaction was warmed to room temperature and was stirred for $5 \mathrm{~h}$. The reaction was quenched with saturated aqueous $\mathrm{NH}_{4} \mathrm{Cl}$ and extracted three times with ethyl acetate. The combined organic layers was washed with brine, dried over anhydrous $\mathrm{Na}_{2} \mathrm{SO}_{4}$, filtered and concentrated. Silica gel chromatography (20\% ethyl acetate-hexanes) afforded vinyl ether $8(277 \mathrm{mg}, 0.97 \mathrm{mmol}, 61 \%$ yield) as $1: 1$ cis- and trans- isomers, along with $0.11 \mathrm{~g}(0.164 \mathrm{mmol}, 27 \%)$ of the starting ketone. ${ }^{1} \mathrm{H}$ NMR $\left(300 \mathrm{MHz}_{,} \mathrm{CDCl}_{3}\right) ; \delta(\mathrm{ppm}): 7.36-7.25(\mathrm{~m}, 10 \mathrm{H}) ; 6.45$ (d, $\mathrm{J}=7.8 \mathrm{~Hz}, 1 \mathrm{H}) ; 6.42(\mathrm{~d}, \mathrm{~J}=7.8 \mathrm{~Hz}, 1 \mathrm{H}) ; 6.15-6.09(\mathrm{~m}, 2 \mathrm{H}) ; 5.95$ ( $\mathrm{t}$, $\mathrm{J}=2.4 \mathrm{~Hz}, 1 \mathrm{H}) ; 5.84$ ( $\mathrm{t}, \mathrm{J}=2.4 \mathrm{~Hz}, 1 \mathrm{H}) ; 4.58$ (d, J=12.0 Hz, $1 \mathrm{H}) ; 4.49$ ( $2 \mathrm{H}) ; 4.48(\mathrm{~d}, \mathrm{~J}=12.0 \mathrm{~Hz}, 1 \mathrm{H}) ; 3.96(\mathrm{dd}, \mathrm{J} 1=6.0 \mathrm{~Hz}, \mathrm{~J} 2=3.0 \mathrm{~Hz}, 1 \mathrm{H})$;

7 Hou, H.-F.; Peddinti, R. K.; Liao, C.-C. Org. Lett. 2002, 4, 2477-2480. 
3.80-3.75 (m, 2H); $3.56(\mathrm{~s}, 3 \mathrm{H}) ; 3.55(\mathrm{~s}, 3 \mathrm{H}) ; 3.40(\mathrm{~s}, 3 \mathrm{H}) ; 3.39$ ( $3 \mathrm{H}) ; 3.14(\mathrm{dd}, \mathrm{J} 1=6.0 \mathrm{~Hz}, \mathrm{~J} 2=3.0 \mathrm{~Hz}, 1 \mathrm{H}) ; 2.15-2.04$ (m, 4H); 2.05-1.93 $(\mathrm{m}, 2 \mathrm{H}) ; 1.64-1.54(\mathrm{~m}, 2 \mathrm{H})$.

To a solution of vinyl ether 8 (226 mg, $0.79 \mathrm{mmol})$ in THF (15 ml) at room temperature was added $10 \mathrm{ml}$ aqueous solution of $\mathrm{HClO}_{4}(65 \%$ $\left.\mathrm{HClO}_{4}: \mathrm{H}_{2} \mathrm{O}=1: 1, \mathrm{~V} / \mathrm{v}\right)$, the reaction mixture was stirred $1.5 \mathrm{hr}$ and then diluted with ethyl acetate, quenched by saturated aqueous $\mathrm{NaHCO}_{3}$ and extracted three times with ethyl acetate. The combined organic layers was washed with brine, dried over anhydrous $\mathrm{Na}_{2} \mathrm{SO}_{4}$, filtered and concentrated. The crude aldehyde was used directly to next step without purification.

To a solution of the above aldehyde in $11.2 \mathrm{~mL}$ of tert-butyl alcohol and $10.5 \mathrm{ml}$ of water at room temperature was added $1.54 \mathrm{~mL}$ of 2-methyl-2-butene. A solution of sodium chlorite (80\%) (0.735 $9,6.50$ mmol) and sodium phosphate monobasic monohydrate (0.710 g, 5.14 mmol) in water was added in portions during $20 \mathrm{~min}$ to the reaction mixture, then stirred for an addition hour. The reaction was poured into $\mathrm{PH} 4.0$ buffer and extracted three times with $\mathrm{CH}_{2} \mathrm{Cl}_{2}$, the combined organic layers was washed with brine, dried over anhydrous $\mathrm{Na}_{2} \mathrm{SO}_{4}$, filtered and concentrated. The crude acid was used directly to next step without purification.

To a solution of the above acid in $7.0 \mathrm{~mL} \mathrm{CH}_{2} \mathrm{Cl}_{2}$ and $1.0 \mathrm{~mL}$ methanol at $0^{\circ} \mathrm{C}$ was added $\operatorname{TMSCHN}_{2}\left(1.0 \mathrm{~mL}\right.$ of $2 \mathrm{M}$ solution in $\mathrm{Et} \mathrm{O}_{2} \mathrm{O}$. The resulting reaction mixture was raised to room temperature and stirred for $1 \mathrm{~h}$. The reaction mixture was then concentrated and subjected to column chromatography (silica, 25\% ethyl acetate-hexanes) to provide $212 \mathrm{mg}(0.708 \mathrm{mmol}, 83 \%$ yield) of esters $1 \mathrm{c}$.

Analytical data for each of the diastereoisomers list below:

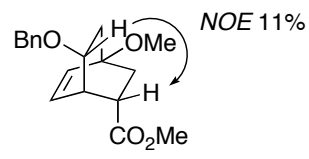

${ }^{1} \mathrm{H} \quad \mathrm{NMR}\left(500 \mathrm{MHz}, \mathrm{CDCl}_{3}\right) ; \delta(\mathrm{ppm}): 7.38-7.26(\mathrm{~m}, 5 \mathrm{H}) ; 6.49(\mathrm{~d}, \mathrm{~J}=8.5 \mathrm{~Hz}$, $1 \mathrm{H}) ; 6.04(\mathrm{dd}, \mathrm{J} 1=8.5 \mathrm{~Hz}, \mathrm{~J} 2=6.0 \mathrm{~Hz}, 1 \mathrm{H}) ; 4.54(\mathrm{~d}, \mathrm{~J}=12.0 \mathrm{~Hz}, 1 \mathrm{H}) ; 4.49$ $(\mathrm{d}, \mathrm{J}=12.0 \mathrm{~Hz}, 1 \mathrm{H}) ; 3.84-3.81(\mathrm{~m}, 1 \mathrm{H}) ; 3.67(\mathrm{~s}, 3 \mathrm{H}) ; 3.40(\mathrm{~s}, 3 \mathrm{H}) ; 3.29$ ( $t, J=3.0 \mathrm{~Hz}, 1 \mathrm{H}$ ); 2.59 (ddd, J1=10.5 Hz, J2=5.0 Hz, J3=2.0 Hz, $1 \mathrm{H}$ ); 1.96-1.88 (m, 2H); $1.65(\mathrm{dd}, \mathrm{J} 1=12.0 \mathrm{~Hz}, \mathrm{~J} 2=10.5 \mathrm{~Hz}, 1 \mathrm{H}) ; 1.57$ (dt, $\left.\mathrm{J}_{\mathrm{d}}=12.5 \mathrm{~Hz}, \mathrm{~J}_{\mathrm{t}}=3.0 \mathrm{~Hz}, 1 \mathrm{H}\right) \cdot{ }^{13} \mathrm{C} \operatorname{NMR}\left(75 \mathrm{MHz}, \mathrm{CDCl}_{3}\right) ; \delta(\mathrm{ppm}): 174.57$, 138.49 , $136.06,128.60,127.75,127.18,77.36,76.63,70.17,52.23$, 
$51.56,39.61,39.42,37.01,32.77$. HRMS (ESI) calcd for $\mathrm{C}_{18} \mathrm{H}_{22} \mathrm{O}_{4} \mathrm{Na}[\mathrm{M}+\mathrm{Na}]$ 325.1416 , found 325.1411 .

Z $\mathrm{CO}_{2} \mathrm{Me}$

${ }^{1} \mathrm{H} \quad \mathrm{NMR}\left(500 \mathrm{MHz}, \mathrm{CDCl}_{3}\right) ; \delta(\mathrm{ppm}): 7.37-7.26(\mathrm{~m}, 5 \mathrm{H}) ; 6.45(\mathrm{~d}, \mathrm{~J}=8.5 \mathrm{~Hz}$, $1 \mathrm{H}) ; 6.16(\mathrm{dd}, \mathrm{J} 1=8.5 \mathrm{~Hz}, \mathrm{~J} 2=6.5 \mathrm{~Hz}, 1 \mathrm{H}) ; 4.46(\mathrm{~s}, 2 \mathrm{H}) ; 3.82-3.79(\mathrm{~m}$, $1 \mathrm{H}) ; 3.65(\mathrm{~s}, 3 \mathrm{H}) ; 3.38(\mathrm{~s}, 3 \mathrm{H}) ; 3.18-3.16(\mathrm{~m}, 1 \mathrm{H}) ; 2.52$ (ddd, J1=11.5 $\mathrm{Hz}, \mathrm{J} 2=5.5 \mathrm{~Hz}, \mathrm{~J} 3=3.0 \mathrm{~Hz}, 1 \mathrm{H}) ; 2.05(\mathrm{dd}, \mathrm{J} 1=12.0 \mathrm{~Hz}, \mathrm{~J} 2=8.5 \mathrm{~Hz}, 1 \mathrm{H}) ;$ $1.94(\mathrm{dd}, \mathrm{J} 1=12.0 \mathrm{~Hz}, \mathrm{~J} 2=5.5 \mathrm{~Hz}, 1 \mathrm{H}) ; 1.62 \mathrm{dt}, \mathrm{J}_{\mathrm{t}}=12.0 \mathrm{~Hz}, \mathrm{~J}_{\mathrm{d}}=4.0 \mathrm{~Hz}$, $1 \mathrm{H}) ; 1.55\left(\mathrm{dt}, \mathrm{J}_{\mathrm{d}}=12.0 \mathrm{~Hz}, \mathrm{~J}_{\mathrm{t}}=3.5 \mathrm{~Hz}, 1 \mathrm{H}\right) \cdot{ }^{13} \mathrm{C} \mathrm{NMR}\left(75 \mathrm{MHz}, \mathrm{CDCl}_{3}\right) ; \delta(\mathrm{ppm})$ : 174.57 , $138.56,136.58,129.23,128.51,127.82,127.73,77.66,73.94$, $70.26,52.20,51.31,40.58,39.70,36.81,31.28$. HRMS (ESI) calcd for $\mathrm{C}_{18} \mathrm{H}_{22} \mathrm{O}_{4} \mathrm{Na}$ [M+Na] 325.1416 , found 325.1411 .

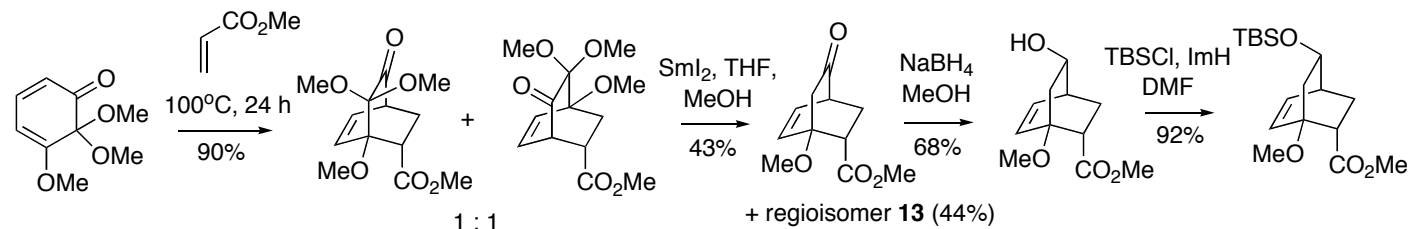
9 $10 \quad 1: 1 \quad 11$ 12

14

$1 f$

Cycloaddition products 10+11. A mixture of 2,4-cyclohexadienone $\mathbf{9}^{1}$ (0.92 $\mathrm{g}, 5 \mathrm{mmol})$ and methyl acrylate $(4.5 \mathrm{ml}, 50 \mathrm{mmol})$ was kept in a sealed tube at $100^{\circ} \mathrm{C}$ for $24 \mathrm{~h}$. The reaction mixture was then cooled to room temperature and subjected to column chromatography (silica, 20\%

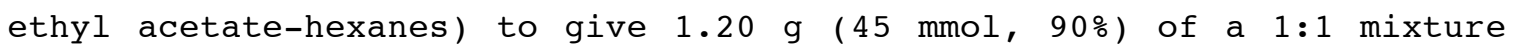
of the two regioisomeric Diels-Alder reaction products $10+11$. Analytical sample of each isomer was separated by column chromatography (silica, 1.5\% THF-benzene). Considering the separation is difficult at this stage it is suggested to separate the regioisomers at next step.

Analytical data for 10: ${ }^{1} \mathrm{H} \operatorname{NMR}\left(300 \mathrm{MHz}, \mathrm{CDCl}_{3}\right) ; \delta(\mathrm{ppm}): 6.51$ (d, $\mathrm{J}=8.4 \mathrm{~Hz}, 1 \mathrm{H}) ; 6.18(\mathrm{dd}, \mathrm{J} 1=8.4 \mathrm{~Hz}, \mathrm{~J} 2=6.6 \mathrm{~Hz}, 1 \mathrm{H}) ; 3.68(\mathrm{~s}, 3 \mathrm{H}) ; 3.65$ $(\mathrm{s}, 3 \mathrm{H}) ; 3.53(\mathrm{~s}, 3 \mathrm{H}) ; 3.55-3.52(\mathrm{~m}, 1 \mathrm{H}) ; 3.42(\mathrm{~s}, 3 \mathrm{H}) ; 3.17-3.14(\mathrm{~m}$, $1 \mathrm{H}) ; 2.17-2.08(\mathrm{~m}, 1 \mathrm{H}) ; 1.82$ (ddd, J1=13.2 Hz, J2=4.8 Hz, J3=3.6 Hz, $1 \mathrm{H}) \cdot{ }^{13} \mathrm{C} \operatorname{NMR}\left(75 \mathrm{MHz}, \mathrm{CDCl}_{3}\right) ; \delta(\mathrm{ppm}): 199.57,173.84,133.84,123.75$, $95.63,84.18,54.33,52.45,51.70,51.36,46.51,41.63,26.96$. HRMS (ESI) calcd for $\mathrm{C}_{13} \mathrm{H}_{18} \mathrm{O}_{6} \mathrm{Na}$ [M+Na] 293.1001, found 293.1005.

Analytical data for 11: ${ }^{1} \mathrm{H} \operatorname{NMR}\left(300 \mathrm{MHz}, \mathrm{CDCl}_{3}\right) ; \delta(\mathrm{ppm}): 6.25$ (d, $\mathrm{J}=8.4 \mathrm{~Hz}, 1 \mathrm{H}) ; 6.06(\mathrm{dd}, \mathrm{J} 1=8.4 \mathrm{~Hz}, \mathrm{~J} 2=6.0 \mathrm{~Hz}, 1 \mathrm{H}) ; 3.70(\mathrm{~s}, 3 \mathrm{H}) ; 3.51$ $(\mathrm{s}, 3 \mathrm{H}) ; 3.49(\mathrm{~s}, 3 \mathrm{H}) ; 3.47(\mathrm{~s}, 3 \mathrm{H}) ; 3.52-3.46(\mathrm{~m}, 1 \mathrm{H}) ; 3.02-2.96(\mathrm{~m}$, 
$1 \mathrm{H}) ; 2.37(\mathrm{dd}, \mathrm{J} 1=12.3 \mathrm{~Hz}, \mathrm{~J} 2=10.2 \mathrm{~Hz}, 1 \mathrm{H}) ; 2.12$ ( dd, J1=12.3 $\mathrm{Hz}$, $\mathrm{J} 2=5.4 \mathrm{~Hz}, 1 \mathrm{H}) \cdot{ }^{13} \mathrm{C} \operatorname{NMR}\left(75 \mathrm{MHz}, \mathrm{CDCl}_{3}\right) ; \delta(\mathrm{ppm}): 198.79,172.82,137.49$, $122.83,94.07,83.00,52.50,52.34,51.94,51.85,48.76,37.02,27.68$. HRMS (ESI) calcd for $\mathrm{C}_{13} \mathrm{H}_{18} \mathrm{O}_{6} \mathrm{Na}$ [M+Na] 293.1001, found 293.1001.

Ketones 12+13. To a solution of $\alpha, \alpha$-dimethoxy-ketone $10+11$ (500 mg, $1.85 \mathrm{mmol})$ in methanol $(2 \mathrm{~mL})$ was added $\mathrm{SmI}_{2}$ solution $(0.1 \mathrm{M}$ in $\mathrm{THF})$ (70 $\mathrm{mL}, 7 \mathrm{mmol}$ ) at room temperature until the blue color of the reaction mixture did not faded. The mixture was stirred for 10 min and then diluted with ethyl acetate, washed with solution of $\mathrm{K}_{2} \mathrm{CO}_{3}$. The organic layer was separated and the aqueous layer was extracted three times with ethyl acetate. The combined organic layers was washed with brine, dried over anhydrous $\mathrm{Na}_{2} \mathrm{SO}_{4}$, filtered and concentrated. Silica gel chromatography (20\% ethyl acetate-hexanes) afforded reduction products 12 (168 mg, $0.80 \mathrm{mmol}, 43 \% \mathrm{yield})$ and 13 (172 mg, $0.82 \mathrm{mmol}, 44 \%$ yield). Analytical data for 12: ${ }^{1} \mathrm{H} \operatorname{NMR}\left(300 \mathrm{MHz}, \mathrm{CDCl}_{3}\right) ; \delta(\mathrm{ppm}): 6.42$ (d, $\mathrm{J}=9.0 \mathrm{~Hz}, 1 \mathrm{H}) ; 6.27(\mathrm{dd}, \mathrm{J} 1=9.0 \mathrm{~Hz}, \mathrm{~J} 2=6.0 \mathrm{~Hz}, 1 \mathrm{H}) ; 3.70(\mathrm{~s}, 3 \mathrm{H}) ; 3.42$ $(\mathrm{s}, 3 \mathrm{H}) ; 3.21-3.16(\mathrm{~m}, 1 \mathrm{H}) ; 3.12(\mathrm{dd}, \mathrm{J} 1=9.6 \mathrm{~Hz}, \mathrm{~J} 2=5.4 \mathrm{~Hz}, 1 \mathrm{H}) ; 2.38$ $(\mathrm{d}, \mathrm{J}=17.4 \mathrm{~Hz}, 1 \mathrm{H}) ; 2.22(\mathrm{~d}, \mathrm{~J}=17.4 \mathrm{~Hz}, 1 \mathrm{H}) ; 2.12-2.03$ (m, $1 \mathrm{H}) ; 1.87$ ( ddd, J1=13.2 Hz, J2=5.4 Hz, J3=3.0 Hz, $1 \mathrm{H}) .{ }^{13} \mathrm{C} \mathrm{NMR}\left(75 \mathrm{MHz}^{\mathrm{CDCl}} \mathrm{C}_{3}\right)$; $\delta(\mathrm{ppm}): 206.88,173.49,135.90,126.23,79.94,51.83,51.33,47.72$, $45.36,42.94,27.78$. HRMS (ESI) calcd for $\mathrm{C}_{11} \mathrm{H}_{14} \mathrm{O}_{4} \mathrm{Na}$ [M+Na] 233.0790, found 233.0782 .

Analytical data for 13: ${ }^{1} \mathrm{H} \operatorname{NMR}\left(300 \mathrm{MHz}, \mathrm{CDCl}_{3}\right) ; \delta(\mathrm{ppm}): 6.58$ (d, $\mathrm{J}=8.4 \mathrm{~Hz}, 1 \mathrm{H}) ; 6.09(\mathrm{dd}, \mathrm{J} 1=8.4 \mathrm{~Hz}, \mathrm{~J} 2=6.0 \mathrm{~Hz}, 1 \mathrm{H}) ; 3.70(\mathrm{~s}, 3 \mathrm{H}) ; 3.49$ ( ddd, J1=6.0 Hz, J2=2.4 Hz, J3=1.2 Hz, 1H); 3.43 ( s, 3H); 2.95 (ddd, $\mathrm{J} 1=10.2 \mathrm{~Hz}, \mathrm{~J} 2=5.4 \mathrm{~Hz}, \mathrm{~J} 3=2.4 \mathrm{~Hz}, 1 \mathrm{H}) ; 2.35$ (dd, J1=17.4 Hz, J2=3.6 Hz, $1 \mathrm{H}) ; 2.20(\mathrm{~d}, \mathrm{~J}=17.4 \mathrm{~Hz}, 1 \mathrm{H}) ; 2.16$ (ddd, J1=12.6 Hz, J2=5.4 Hz, J3=3.6 $\mathrm{Hz}, 1 \mathrm{H}) ; 2.07(\mathrm{dd}, \mathrm{J} 1=12.6 \mathrm{~Hz}, \mathrm{~J} 2=10.2 \mathrm{~Hz}, 1 \mathrm{H}) .{ }^{13} \mathrm{C} \mathrm{NMR}\left(75 \mathrm{MHz} \mathrm{CDCl}_{3}\right)$; $\delta(\mathrm{ppm}): 206.16,172.79,138.75,123.93,77.84,52.34,51.34,50.25$, $43.34,38.08,32.74$. HRMS (CI) calcd for $\mathrm{C}_{11} \mathrm{H}_{15} \mathrm{O}_{4}[\mathrm{M}+\mathrm{H}] 211.0970$, found 211.0977 .

Alcohol 14. To a solution of ketone 12 (150 $\mathrm{mg}, 0.71 \mathrm{mmol})$ in methanol (2 mL) was added $\mathrm{NaBH}_{4}(19 \mathrm{mg}, 0.50 \mathrm{mmol})$ at room temperature. The mixture was stirred for $10 \mathrm{~min}$ and then concentrated, ethyl acetate and 5\% HCl was added. The organic layer was separated and the aqueous layer was extracted three times with ethyl acetate. The combined organic layers was washed with brine, dried over anhydrous $\mathrm{Na}_{2} \mathrm{SO}_{4}$, filtered and concentrated. Silica gel chromatography (30\% to 50\% ethyl 
acetate-hexanes) afforded alcohol 14 (102 mg, $0.48 \mathrm{mmol}$, 68\% yield) and its diastereoisomer (36 mg, $0.17 \mathrm{mmol}, 24 \%$ yield).

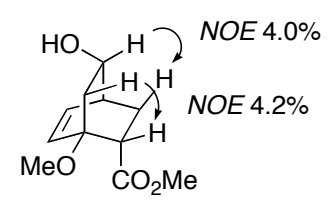

Alcohol 14: ${ }^{1} \mathrm{H}$ NMR (300 $\left.\mathrm{MHz}, \mathrm{CDCl}_{3}\right) ; \delta(\mathrm{ppm}): 6.40$ (d, J=9.0 $\mathrm{Hz}$, $\left.1 \mathrm{H}\right)$; $6.20(\mathrm{dd}, \mathrm{J} 1=9.0 \mathrm{~Hz}, \mathrm{~J} 2=6.6 \mathrm{~Hz}, 1 \mathrm{H}) ; 4.06-4.03(\mathrm{~m}, 1 \mathrm{H}) ; 3.65(\mathrm{~s}, 3 \mathrm{H})$; $3.38(\mathrm{~s}, 3 \mathrm{H}) ; 2.80-2.76(\mathrm{~m}, 1 \mathrm{H}) ; 2.71(\mathrm{dd}, \mathrm{J} 1=9.6 \mathrm{~Hz}, \mathrm{~J} 2=5.4 \mathrm{~Hz}, 1 \mathrm{H})$; $2.11(\mathrm{dd}, \mathrm{J} 1=12.9 \mathrm{~Hz}, \mathrm{~J} 2=8.4 \mathrm{~Hz}, 1 \mathrm{H}) ; 1.81$ (ddd, J1=13.2 Hz, J2=10.2 Hz, $\mathrm{J} 3=2.4 \mathrm{~Hz}, 1 \mathrm{H}) ; 1.59$ (ddd, J1=13.2 Hz, J2=4.8 Hz, J3=3.0 Hz, 1H); $1.50-$ 1.46 (br, $1 \mathrm{H},-\underline{\mathrm{H}}), 1.42(\mathrm{dd}, \mathrm{J} 1=12.9 \mathrm{~Hz}, \mathrm{~J} 2=3.0 \mathrm{~Hz}, 1 \mathrm{H}) .{ }^{13} \mathrm{C} \mathrm{NMR}$ (75 $\left.\mathrm{MHz}, \mathrm{CDCl}_{3}\right) ; \delta(\mathrm{ppm}): 174.62,134.08,128.94,78.88,69.33,51.69,51.09$, $45.03,41.36,37.53,28.24$. HRMS (ESI) calcd for $\mathrm{C}_{11} \mathrm{H}_{16} \mathrm{O}_{4} \mathrm{Na}$ [M+Na] 235.0946 , found 235.0948 .

TBS ether 1f: To a solution of alcohol 14 (80 mg, $0.38 \mathrm{mmol})$ in dry DMF ( $3 \mathrm{~mL}$ ) was added imidazole $(51 \mathrm{mg}, 0.76 \mathrm{mmol})$, DMAP ( $5 \mathrm{mg}, 0.038 \mathrm{mmol})$ and TBSCl (85 mg, $0.56 \mathrm{mmol}$ ) at room temperature. The mixture was stirred for $12 \mathrm{hr}$ and then poured to ethyl acetate, washed with brine, dried over anhydrous $\mathrm{Na}_{2} \mathrm{SO}_{4}$, filtered and concentrated. Silica gel chromatography (10\% ethyl acetate-hexanes) afforded TBS ether $1 \mathrm{f}$ (114 $\mathrm{mg}, 0.35 \mathrm{mmol}, 92 \%$ yield). ${ }^{1} \mathrm{H} \mathrm{NMR}\left(300 \mathrm{MHz}, \mathrm{CDCl}_{3}\right) ; \delta(\mathrm{ppm}): 6.31$ (d, $\mathrm{J}=8.4 \mathrm{~Hz}, 1 \mathrm{H}) ; 6.20$ ( dd, J1=8.4 Hz, J2=6.0 Hz, $1 \mathrm{H}) ; 4.03-3.98$ (m, $1 \mathrm{H})$; $3.65(\mathrm{~s}, 3 \mathrm{H}) ; 3.38(\mathrm{~s}, 3 \mathrm{H}) ; 2.67(\mathrm{dd}, \mathrm{J} 1=10.2 \mathrm{~Hz}, \mathrm{~J} 2=5.4 \mathrm{~Hz}, 1 \mathrm{H}) ; 2.61$ $(\mathrm{dd}, \mathrm{J} 1=6.0 \mathrm{~Hz}, \mathrm{~J} 2=3.0 \mathrm{~Hz}, 1 \mathrm{H}) ; 1.98(\mathrm{dd}, \mathrm{J} 1=12.6 \mathrm{~Hz}, \mathrm{~J} 2=8.4 \mathrm{~Hz}, 1 \mathrm{H})$; 1.76 (ddd, J1=13.2 Hz, J2=10.2 Hz, J3=3.0, 1H); 1.53 ( $\mathrm{J} 2=5.4 \mathrm{~Hz}, \mathrm{~J} 3=3.0,1 \mathrm{H}) ; 1.44(\mathrm{dd}, \mathrm{J} 1=11.7 \mathrm{~Hz}, \mathrm{~J} 2=3.0,1 \mathrm{H}) ; 0.85$ ( $\mathrm{s}, 9 \mathrm{H})$; $0.04(\mathrm{~s}, 3 \mathrm{H}) ; 0.03(\mathrm{~s}, 3 \mathrm{H}) \cdot{ }^{13} \mathrm{C} \operatorname{NMR}\left(75 \mathrm{MHz}, \mathrm{CDCl}_{3}\right) ; \delta(\mathrm{ppm}): 174.83$, $132.12,129.79,78.86,70.00,51.61,51.13,45.15,41.95,37.87,28.47$, 25.75, 17.99, -4.65, -4.77. HRMS (ESI) Calcd for $\mathrm{C}_{17} \mathrm{H}_{30} \mathrm{O}_{4} \mathrm{SiNa}$ [M+Na] 349.1811 , found 349.1824 . 


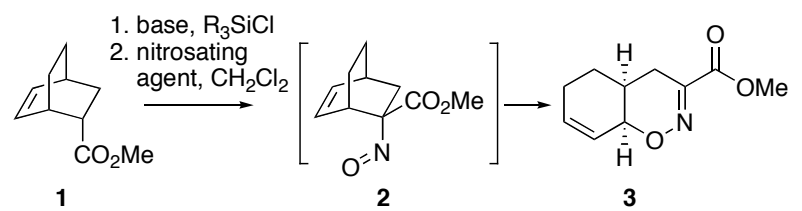

Nitrosation/1,2-oxaza-Cope rearrangement $1 \rightarrow 3$ using nitrosonium hexafluoroantimonate.

To a solution of LDA [from $i-\mathrm{Pr}_{2} \mathrm{NH}(79 \mu \mathrm{l}, 0.564 \mathrm{mmol})$ and $n-\mathrm{BuLi}$ (2.65 $M$ in hexanes, $0.19 \mathrm{ml}, 0.50 \mathrm{mmol})$, in $\operatorname{THF}(0.75 \mathrm{ml}),-78^{\circ} \mathrm{C}, 30$ min] was added a solution of ester $1(40 \mathrm{mg}, 0.241 \mathrm{mmol})$ in THF (0.75 ml total with rinses) at $-78^{\circ} \mathrm{C}$. After stirring for $30 \mathrm{~min}$, TMSCl $(69 \mu 1,0.54$ mmol) was added dropwise at $-78^{\circ} \mathrm{C}$ and, after $30 \mathrm{~min}$, the cooling bath was removed and the reaction mixture was diluted with hexanes and quenched with saturated aqueous sodium bicarbonate. The layers were separated, the organic layer was dried with anhydrous sodium sulfate, filtered and concentrated. The silyl ketene acetal was dissolved in 1.6 ml of dry dichloromethane and triethylamine (67 $\mu l, 0.48$ mmol) was added. The reaction mixture was cooled to $-15^{\circ} \mathrm{C}$, followed by the dropwise addition of a solution of $\mathrm{NOSbF}_{6}(70 \mathrm{mg}, 0.263 \mathrm{mmol})$ in dry nitromethane $(0.40 \mathrm{ml})$. The reaction mixture immediately turned brilliant green. The solution was stirred for $1 \mathrm{~h}$, during which time it warmed to $0^{\circ} \mathrm{C}$. The reaction mixture was quenched with saturated aqueous sodium bicarbonate and extracted with dichloromethane $(2 \times 5 \mathrm{ml})$. The combined organic layers were washed with water, dried with anhydrous sodium sulfate, filtered and concentrated. The residue was purified by column chromatography (silica, 10 $\rightarrow 40 \%$ ethyl acetate-hexanes) to afford $14 \mathrm{mg}$ (0.084 mmol, 35\%) of ester 1 and its diastereomer and oxazine 3 (14 mg, $0.071 \mathrm{mmol}, 30 \%)$.

Nitrosation/1,2-oxaza-Cope rearrangement $1 \rightarrow 3$ using isoamyl nitrite/ $\mathrm{TiCl}_{4}$. General Procedure.

To a solution of LDA [from $i-\mathrm{Pr}_{2} \mathrm{NH}(0.16 \mathrm{ml}, 1.11 \mathrm{mmol})$ and $n-B u L i$ (2.65 $M$ in hexanes, $0.38 \mathrm{ml}, 1.01 \mathrm{mmol})$, in THF $(1.5 \mathrm{ml}),-78^{\circ} \mathrm{C}, 30$ min] was added a solution of ester $1(80 \mathrm{mg}, 0.48 \mathrm{mmol})$ in THF (1.25 ml total with rinses) at $-78^{\circ} \mathrm{C}$. After stirring for $30 \mathrm{~min}$, TMSCl $(0.14 \mathrm{ml}, 1.10$ mmol) was added dropwise at $-78^{\circ} \mathrm{C}$ and, after $30 \mathrm{~min}$, the cooling bath was removed and the reaction mixture was diluted with hexanes and quenched with saturated aqueous sodium bicarbonate. The layers were separated, the organic layer was dried with anhydrous sodium sulfate, filtered and concentrated. The silyl ketene acetal was dissolved in 3.2 
$\mathrm{ml}$ of dry dichloromethane and 2,6-di-tert-butyl-4-methylpyridine (30 mg, $0.146 \mathrm{mmol})$ was added. The reaction mixture was cooled to $-45^{\circ} \mathrm{C}$ and isoamyl nitrite $(77 \mu \mathrm{l}, 0.575 \mathrm{mmol})$ was added, followed by a dropwise addition of a solution of titanium tetrachloride in $\mathrm{CH}_{2} \mathrm{Cl}_{2}(2.0 \mathrm{M}, 0.24$ $\mathrm{ml}, 0.48 \mathrm{mmol})$. The stirred mixture turned bluish green and was allowed to warm to $-15{ }^{\circ} \mathrm{C}$ during $1.5 \mathrm{~h}$. The reaction was quenched by the addition of saturated ammonium chloride. The mixture was extracted with $\mathrm{CH}_{2} \mathrm{Cl}_{2}(2 \times 10 \mathrm{ml})$, and the combined organic layers were dried with anhydrous sodium sulfate, filtered and concentrated. Purification by column chromatography (silica, 35\% ethyl acetate-hexanes) afforded oxazine 3 (67 mg, $0.343 \mathrm{mmol}, 71 \%$ ) as a white crystalline solid.

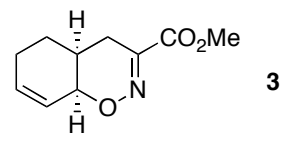

3: $\mathrm{mp}: 86-87^{\circ} \mathrm{C}$; IR $\left(\mathrm{film}, \mathrm{cm}^{-1}\right): 2950,2348,1714,1602,1516,1444,1292$, $1216,1142,1080,990,738,694 ;{ }^{1} \mathrm{H} \operatorname{NMR}\left(300 \mathrm{MHz}, \mathrm{CDCl}_{3}\right) ; \delta(\mathrm{ppm}): 6.01$ ( $d t, J d=10.2 \mathrm{~Hz}, J t=3.6 \mathrm{~Hz}, 1 \mathrm{H}) ; 5.84(\mathrm{dq}, \mathrm{Jd}=10.2 \mathrm{~Hz}, \mathrm{Jq}=2.4 \mathrm{~Hz}, 1 \mathrm{H})$; $4.24(\mathrm{br}, 1 \mathrm{H}) ; 3.84(\mathrm{~s}, 3 \mathrm{H}) ; 2.60(\mathrm{dd}, \mathrm{J} 1=19.8 \mathrm{~Hz}, \mathrm{~J} 2=7.8 \mathrm{~Hz}, 1 \mathrm{H}) ; 2.28$ $(\mathrm{dd}, \mathrm{J} 1=19.8 \mathrm{~Hz}, \mathrm{~J} 2=3.6 \mathrm{~Hz}, 1 \mathrm{H}) ; 2.19-2.11(\mathrm{~m}, 3 \mathrm{H}) ; 1.63-1.56(\mathrm{~m}, 2 \mathrm{H})$. ${ }^{13} \mathrm{C}$ NMR $\left(75 \mathrm{MHz}, \mathrm{CDCl}_{3}\right) ; \delta(\mathrm{ppm}): 164.43,149.02,134.44,124.11,71.29$, $52.85,27.10,25.29,24.72,23.72$. HRMS (ESI) calcd for $\mathrm{C}_{10} \mathrm{H}_{13} \mathrm{NO}_{3} \mathrm{Na}$ [M+Na] 218.0793 , found 218.0801 .

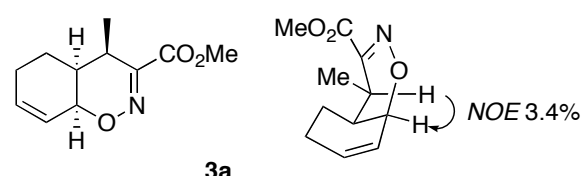

Oxazine 3a. Using the general procedure, ester $1 \mathrm{a}$ ( $81 \mathrm{mg}, 0.45 \mathrm{mmol}$ ) was converted to oxazine $3 \mathrm{a}(75 \mathrm{mg}, 0.36 \mathrm{mmol}, 80 \%$ ) obtained after by column chromatography (silica, 25\% ethyl acetate-hexanes).

3a: IR (film, $\left.\mathrm{cm}^{-1}\right):$ 2938, 2356, 1738, 1720, 1454, 1298, 1214, 1154, $1088,992,736,674 ;{ }^{1} \mathrm{H} \operatorname{NMR}\left(500 \mathrm{MHz}, \mathrm{CDCl}_{3}\right) ; \delta(\mathrm{ppm}): 6.11$ (ddd, J1=10.0 $\mathrm{Hz}, \mathrm{J} 2=5.0 \mathrm{~Hz}, \mathrm{~J} 3=2.5 \mathrm{~Hz},, 1 \mathrm{H}) ; 5.93-5.89(\mathrm{~m}, 1 \mathrm{H}) ; 4.13$ (d, J=5.5, 1H); $3.84(\mathrm{~s}, 3 \mathrm{H}) ; 3.03$ (quint, $J=7.5 \mathrm{~Hz}, 1 \mathrm{H}) ; 2.29$ (dtt, Jd=18.5 Hz, Jt=5.5 $\mathrm{Hz}, \mathrm{Jt}=1.5 \mathrm{~Hz}, 1 \mathrm{H}) ; 2.16-2.08(\mathrm{~m}, 1 \mathrm{H}) ; 2.03$ (ddt, Jd1=13.0 Hz, Jd2=7.5 $\mathrm{Hz}, \mathrm{Jt}=2.5 \mathrm{~Hz}, 1 \mathrm{H}) ; 1.73-1.69(\mathrm{~m}, 1 \mathrm{H}) ; 1.41(\mathrm{dq}, \mathrm{Jq}=13.0 \mathrm{~Hz}, \mathrm{Jd}=6.0 \mathrm{~Hz}$, $1 \mathrm{H}) ; 1.18(\mathrm{~d}, \mathrm{~J} 1=7.5 \mathrm{~Hz}, 3 \mathrm{H}) .{ }^{13} \mathrm{C} \operatorname{NMR}\left(75 \mathrm{MHz}, \mathrm{CDCl}_{3}\right) ; \delta(\mathrm{ppm}): 163.73$, $154.10,135.37,122.73,72.39,52.24,33.77,29.78,25.79,17.70,13.08$. HRMS (ESI) calcd for $\mathrm{C}_{11} \mathrm{H}_{15} \mathrm{NO}_{3} \mathrm{Na}$ [M+Na] 232.0950, found 232.0943 . 


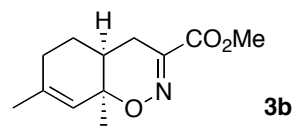

Oxazine 3b. Using the general procedure, ester $1 \mathrm{~b}(93 \mathrm{mg}, 0.48 \mathrm{mmol}$ ) was converted to oxazine $3 \mathbf{b}(80 \mathrm{mg}, 0.36 \mathrm{mmol}, 75 \%)$ obtained as a white crystalline solid after by column chromatography (silica, 25\% ethyl acetate-hexanes).

3b: $\mathrm{mp}: 75-76^{\circ} \mathrm{C}$; IR (film, $\left.\mathrm{Cm}^{-1}\right): 2932,2360,2340,1746,1722,1440$, $1336,1304,1280,1192,1110,1000,964,668 ;{ }^{1} \mathrm{H} \operatorname{NMR}\left(300 \mathrm{MHz}, \mathrm{CDCl}_{3}\right)$; $\delta(\mathrm{ppm}): 5.43(\mathrm{~s}, 1 \mathrm{H}) ; 3.84(\mathrm{~s}, 3 \mathrm{H}) ; 2.59(\mathrm{dd}, \mathrm{J} 1=19.8 \mathrm{~Hz}, \mathrm{~J} 2=7.8 \mathrm{~Hz}, 1 \mathrm{H})$; $2.30(\mathrm{dd}, \mathrm{J} 1=19.8 \mathrm{~Hz}, \mathrm{~J} 2=2.4 \mathrm{~Hz}, 1 \mathrm{H}) ; 2.03(\mathrm{dd}, \mathrm{J} 1=6.6 \mathrm{~Hz}, \mathrm{~J} 2=5.4 \mathrm{~Hz}$ ， $2 \mathrm{H}) ; 1.84-1.80(\mathrm{~m}, 1 \mathrm{H}) ; 1.71(\mathrm{~s}, 3 \mathrm{H}) ; 1.62-1.43(\mathrm{~m}, 2 \mathrm{H}) ; 1.27(\mathrm{~s}, 3 \mathrm{H})$. ${ }^{13} \mathrm{C}$ NMR $\left(75 \mathrm{MHz}, \mathrm{CDCl}_{3}\right) ; \delta(\mathrm{ppm}): 164.61,147.66,140.80,124.70,75.71$, $52.75,31.68,29.64,25.63,24.48,24.40,23.45$. HRMS (ESI) calcd for $\mathrm{C}_{12} \mathrm{H}_{17} \mathrm{NO}_{3} \mathrm{Na}[\mathrm{M}+\mathrm{Na}] 246.1106$, found 246.1107 .

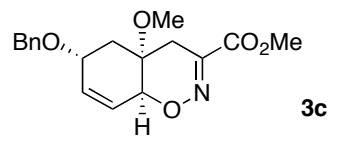

Oxazine 3c. Using the general procedure, esters 1c (exo:endo 1.5:1, 94 $\mathrm{mg}, 0.311 \mathrm{mmol})$ were converted to oxazine 3c (60 mg, $0.193 \mathrm{mmol}, 62 \%)$ obtained after by column chromatography (silica, 20\% ethyl acetatebenzene) .

3c: IR (film, $\left.\mathrm{cm}^{-1}\right):$ 2952, 2356, 1722, 1606, 1722, 1454, 1298, 1214, $1154,1088,992,736,698 ;{ }^{1} \mathrm{H}$ NMR $\left(300 \mathrm{MHz}, \mathrm{CDCl}_{3}\right) ; \delta(\mathrm{ppm}): 7.39-7.27(\mathrm{~m}$, $5 \mathrm{H}) ; 5.95(d t, J d=6.3 \mathrm{~Hz}, J t=1.2 \mathrm{~Hz}, 1 \mathrm{H}) ; 5.82$ (dt, Jd=6.3 Hz, Jt=1.2 $\mathrm{Hz}, 1 \mathrm{H}) ; 4.75$ (quint, $\mathrm{J}=1.2 \mathrm{~Hz}, 1 \mathrm{H}) ; 4.62$ (d, J=7.2 Hz, 1H); 4.59 (d, $\mathrm{J}=7.2 \mathrm{~Hz}, 1 \mathrm{H}) ; 4.17-4.12(\mathrm{~m}, 1 \mathrm{H}) ; 3.86(\mathrm{~s}, 3 \mathrm{H}) ; 3.29(\mathrm{~s}, 3 \mathrm{H}) ; 2.53$ (dd, $\mathrm{J} 1=11.4 \mathrm{~Hz}, \mathrm{~J} 2=1.2 \mathrm{~Hz}, 1 \mathrm{H}) ; 2.32(\mathrm{dd}, \mathrm{J} 1=7.8 \mathrm{~Hz}, \mathrm{~J} 2=4.5 \mathrm{~Hz}, 1 \mathrm{H}) ; 2.28$ $(\mathrm{d}, \mathrm{J}=11.4 \mathrm{~Hz}, 1 \mathrm{H}) ; 2.00(\mathrm{dd}, \mathrm{J} 1=7.8 \mathrm{~Hz}, \mathrm{~J} 2=4.5 \mathrm{~Hz}, 1 \mathrm{H}) .{ }^{13} \mathrm{C} \operatorname{NMR}(75 \mathrm{MHz}$, $\left.\mathrm{CDCl}_{3}\right) ; \delta(\mathrm{ppm}): 163.89,147.95,138.01,131.24,129.54,128.73,128.11$, $127.88,77.44,72.93,71.07,69.43,53.03,49.54,35.03,26.59$. HRMS (ESI) calcd for $\mathrm{C}_{18} \mathrm{H}_{21} \mathrm{NO}_{5} \mathrm{Na}$ [M+Na] 354.1317, found 354.1314 .

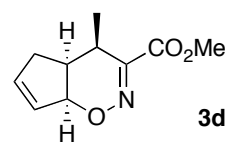

Oxazine 3d. Using the general procedure (exception: the nitrosation/1,2-oxaza-Cope rearrangement were carried out at $-78^{\circ} \mathrm{C}$ for $1 \mathrm{~h})$, ester $1 \mathrm{~d}(0.166 \mathrm{~g}, 1.00 \mathrm{mmol})$ was converted to oxazine $3 \mathrm{~d}$ (98 $\mathrm{mg}$ 
mg, $0.502 \mathrm{mmol}$, 50\%) obtained after by column chromatography (silica, 25\% ethyl acetate-hexanes).

3d: IR (film, $\left.\mathrm{Cm}^{-1}\right): 2966,2358,1756,1560,1412,1218,1142,1078,996$, 748,$690 ;{ }^{1} \mathrm{H} \operatorname{NMR}\left(300 \mathrm{MHz}, \mathrm{CDCl}_{3}\right) ; \delta(\mathrm{ppm}): 6.13(\mathrm{dt}, \mathrm{Jd}=5.4 \mathrm{~Hz}, \mathrm{Jt}=2.4 \mathrm{~Hz}$, $1 \mathrm{H}$ ) ; 5.90 (ddd, J1=5.4 Hz, J2=4.2 Hz, J3=2.4 Hz, 1H); 4.91 (ddd, J1=6.6 $\mathrm{Hz}, \mathrm{J} 2=2.4 \mathrm{~Hz}, \mathrm{~J} 3=1.2 \mathrm{~Hz}, 1 \mathrm{H}) ; 3.87(\mathrm{~s}, 3 \mathrm{H}) ; 3.03$ (quint, J=7.5 Hz, 1H); $2.82(\mathrm{dq}, \mathrm{Jq}=7.2 \mathrm{~Hz}, \mathrm{Jd}=0.6 \mathrm{~Hz}, 1 \mathrm{H}) ; 2.47-2.41(\mathrm{~m}, 2 \mathrm{H}) ; 1.18(\mathrm{~d}, \mathrm{~J}=7.2$ $\mathrm{Hz}, 3 \mathrm{H}) \cdot{ }^{13} \mathrm{C} \mathrm{NMR}\left(75 \mathrm{MHz}, \mathrm{CDCl}_{3}\right) ; \delta(\mathrm{ppm}): 163.91,163.86,137.99,129.70$, 85.53 , 52.92, 40.75, 34.32, 27.91, 13.68. HRMS (ESI) calcd for $\mathrm{C}_{10} \mathrm{H}_{13} \mathrm{NO}_{3} \mathrm{Na}[\mathrm{M}+\mathrm{Na}] 218.0793$, found 218.0799 .

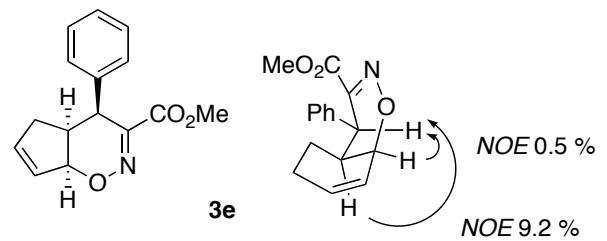

Oxazine 3e. Using the general procedure (exception: the nitrosation/1,2-oxaza-Cope rearrangement were carried out at $-78^{\circ} \mathrm{C}$ for $1 \mathrm{~h})$, ester 1e $(0.19 \mathrm{~g}, 0.83 \mathrm{mmol})$ was converted to oxazine $3 \mathrm{e}(0.143 \mathrm{mg}$ mg, 0.556 mmol, 67\%) obtained as a white crystalline solid after by column chromatography (silica, 25\% ethyl acetate-hexanes).

3e: mp: $84-85{ }^{\circ} \mathrm{C}$; IR (film, $\left.\mathrm{cm}^{-1}\right): 2932,2360,2340,1746,1722,1440$,

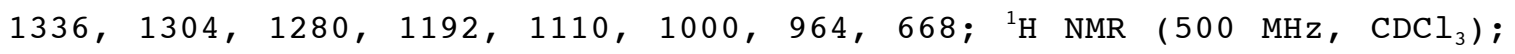
$\delta(\mathrm{ppm}): 7.29-7.22(\mathrm{~m}, 3 \mathrm{H}) ; 7.16-7.14(\mathrm{~m}, 2 \mathrm{H}) ; 6.10 \quad(\mathrm{dt}, \mathrm{Jd}=6.0 \mathrm{~Hz}$, $\mathrm{Jt}=2.0 \mathrm{~Hz}, 1 \mathrm{H}) ; 5.95$ (ddd, J1=6.0 Hz, J2=J3=2.0 Hz, $1 \mathrm{H}) ; 4.67$ (ddd, $\mathrm{J} 1=6.0 \mathrm{~Hz}, \mathrm{~J} 2=\mathrm{J} 3=2.0 \mathrm{~Hz}, 1 \mathrm{H}) ; 4.42(\mathrm{~d}, \mathrm{~J}=10.0 \mathrm{~Hz}, 1 \mathrm{H}) ; 3.76$ ( $\mathrm{s}, 3 \mathrm{H})$; 2.95-2.89 (m, 1H); 2.09 (ddt, Jdl=17.5 Hz, Jd2=8.5 Hz, Jt=2.0 Hz, $1 \mathrm{H})$; 2.02 (ddq, Jd1=17.5 Hz, Jd2=7.5 Hz, Jq=2.5 Hz, $1 \mathrm{H}$ ). ${ }^{13} \mathrm{C} \operatorname{NMR}(75 \mathrm{MHz}$, $\left.\mathrm{CDCl}_{3}\right) ; \delta(\mathrm{ppm}): 163.74,158.81,139.86,137.92,128.92,128.72,128.07$, 127.53, 83.26, 53.00, 40.06, 39.05, 35.25. HRMS (ESI) calcd for $\mathrm{C}_{15} \mathrm{H}_{15} \mathrm{NO}_{3} \mathrm{Na}[\mathrm{M}+\mathrm{Na}] 280.0950$, found 280.0946 .
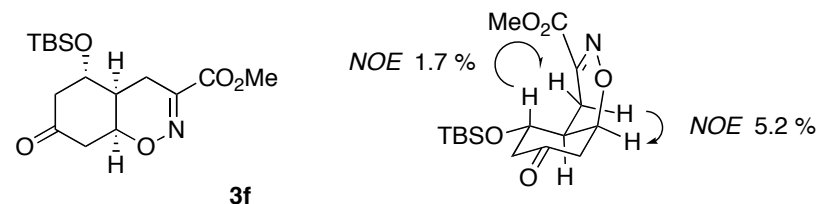

Oxazine 3f. Using the general procedure, ester $1 \mathrm{f}(95 \mathrm{mg}, 0.29 \mathrm{mmol})$ was converted to oxazine $3 \mathbf{f}(60 \mathrm{mg}, 0.175 \mathrm{mmol}, 61 \%)$ obtained as a 
white crystalline solid after by column chromatography (silica, $40 \rightarrow 60 \%$ ethyl acetate-hexanes) .

3f: mp: $102-104{ }^{\circ} \mathrm{C} ;{ }^{1} \mathrm{H}$ NMR $\left(500 \mathrm{MHz}, \mathrm{CDCl}_{3}\right) ; \delta(\mathrm{ppm}): 4.36-4.34(\mathrm{~m}, 1 \mathrm{H})$; $3.88(\mathrm{~s}, 3 \mathrm{H}) ; 3.73\left(\mathrm{dt}, \mathrm{J}_{\mathrm{t}}=10.0 \mathrm{~Hz}, \mathrm{~J}_{\mathrm{d}}=4.5 \mathrm{~Hz}, 1 \mathrm{H}\right) ; 2.95(\mathrm{~d}, \mathrm{~J}=19.5 \mathrm{~Hz}$, $1 \mathrm{H}) ; 2.83\left(\mathrm{dt}, \mathrm{J}_{\mathrm{d}}=16.0 \mathrm{~Hz}, \mathrm{~J}_{\mathrm{t}}=2.5 \mathrm{~Hz}, 1 \mathrm{H}\right) ; 2.74$ (ddd, $\mathrm{J}_{1}=14.0 \mathrm{~Hz}, \mathrm{~J}_{2}=4.5$ $\left.\mathrm{Hz}, \mathrm{J}_{3}=2.5 \mathrm{~Hz}, 1 \mathrm{H}\right) ; 2.60$ (dd, $\left.\mathrm{J}_{1}=16.0 \mathrm{~Hz}, \mathrm{~J}_{2}=4.0 \mathrm{~Hz}, 1 \mathrm{H}\right) ; 2.54$ (dd, $\left.\mathrm{J}_{1}=19.5 \mathrm{~Hz}, \mathrm{~J}_{2}=7.0 \mathrm{~Hz}, 1 \mathrm{H}\right) ; 2.44\left(\mathrm{dd}, \mathrm{J}_{1}=14.0 \mathrm{~Hz}, \mathrm{~J}_{2}=10.0 \mathrm{~Hz}, 1 \mathrm{H}\right) ; 2.32$ ( ddt, Jd1 $=10.0 \mathrm{~Hz}, J d 2=7.5 \mathrm{~Hz}, J t=2.0 \mathrm{~Hz}, 1 \mathrm{H}) ; 0.88(\mathrm{~s}, 9 \mathrm{H}) ; 0.03$ ( $\mathrm{s}$, $3 \mathrm{H}) ; 0.01(\mathrm{~s}, 3 \mathrm{H}) \cdot{ }^{13} \mathrm{C} \operatorname{NMR}\left(75 \mathrm{MHz}, \mathrm{CDCl}_{3}\right) ; \delta(\mathrm{ppm}): 204.75,163.82$, $149.23 ， 74.12,67.68,53.10 ， 50.27,43.92,36.74,25.85,23.44,18.02$, $-4.19,-4.96$. HRMS (ESI) calcd for $\mathrm{C}_{16} \mathrm{H}_{27} \mathrm{NO}_{5} \mathrm{SiNa}$ [M+Na] 364.1556, found 364.1555 .

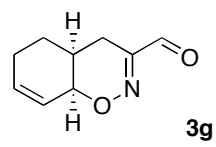

Oxazine 3g. Using the general procedure (exception: the silyl enol ether was prepared by treatment of the aldehyde with TESOTf ( 3 eq) and triethylamine $(10 \mathrm{eq})$ in $\mathrm{dry} \mathrm{CH}_{2} \mathrm{Cl}_{2}(1.0 \mathrm{ml})$ at $\left.\mathrm{rt}, 30 \mathrm{~min}\right)$, aldehyde $\mathbf{1 g}$

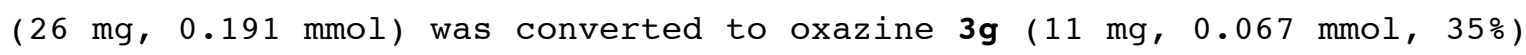
obtained after by column chromatography (silica, 15\% ethyl acetatehexanes).

3g: ${ }^{1} \mathrm{H} \operatorname{NMR}\left(300 \mathrm{MHz}, \mathrm{CDCl}_{3}\right) ; \delta(\mathrm{ppm}): 9.54(\mathrm{~s}, 1 \mathrm{H}) ; 6.07\left(\mathrm{dt}, \mathrm{J}_{\mathrm{d}}=10.2 \mathrm{~Hz}\right.$, $\mathrm{J}_{\mathrm{t}}=3.6 \mathrm{~Hz}, 1 \mathrm{H}$ ); 5.90 (dquint, $\mathrm{Jd}=10.2 \mathrm{~Hz}, \mathrm{Jq}=2.4 \mathrm{~Hz}, 1 \mathrm{H}$ ); 4.36 (br, 1H); $2.48(\mathrm{dd}, \mathrm{J} 1=19.2 \mathrm{~Hz}, \mathrm{~J} 2=7.8 \mathrm{~Hz}, 1 \mathrm{H}) ; 2.21$ (dd, J1=19.2 Hz, J2=3.0 Hz, $1 \mathrm{H}) ; 2.22-2.13(\mathrm{~m}, 3 \mathrm{H}) ; 1.62-1.52(\mathrm{~m}, 2 \mathrm{H}) .{ }^{13} \mathrm{C} \operatorname{NMR}\left(75 \mathrm{MHz}, \mathrm{CDCl}_{3}\right)$; $\delta(\mathrm{ppm}): 191.25,156.16,134.76,123.87,72.99,24.46,26.46,24.86$, 23.64, 22.21. HRMS (CI) calcd for $\mathrm{C}_{9} \mathrm{H}_{12} \mathrm{NO}_{2}$ [M+Na] 166.0868, found 166.0866 . 


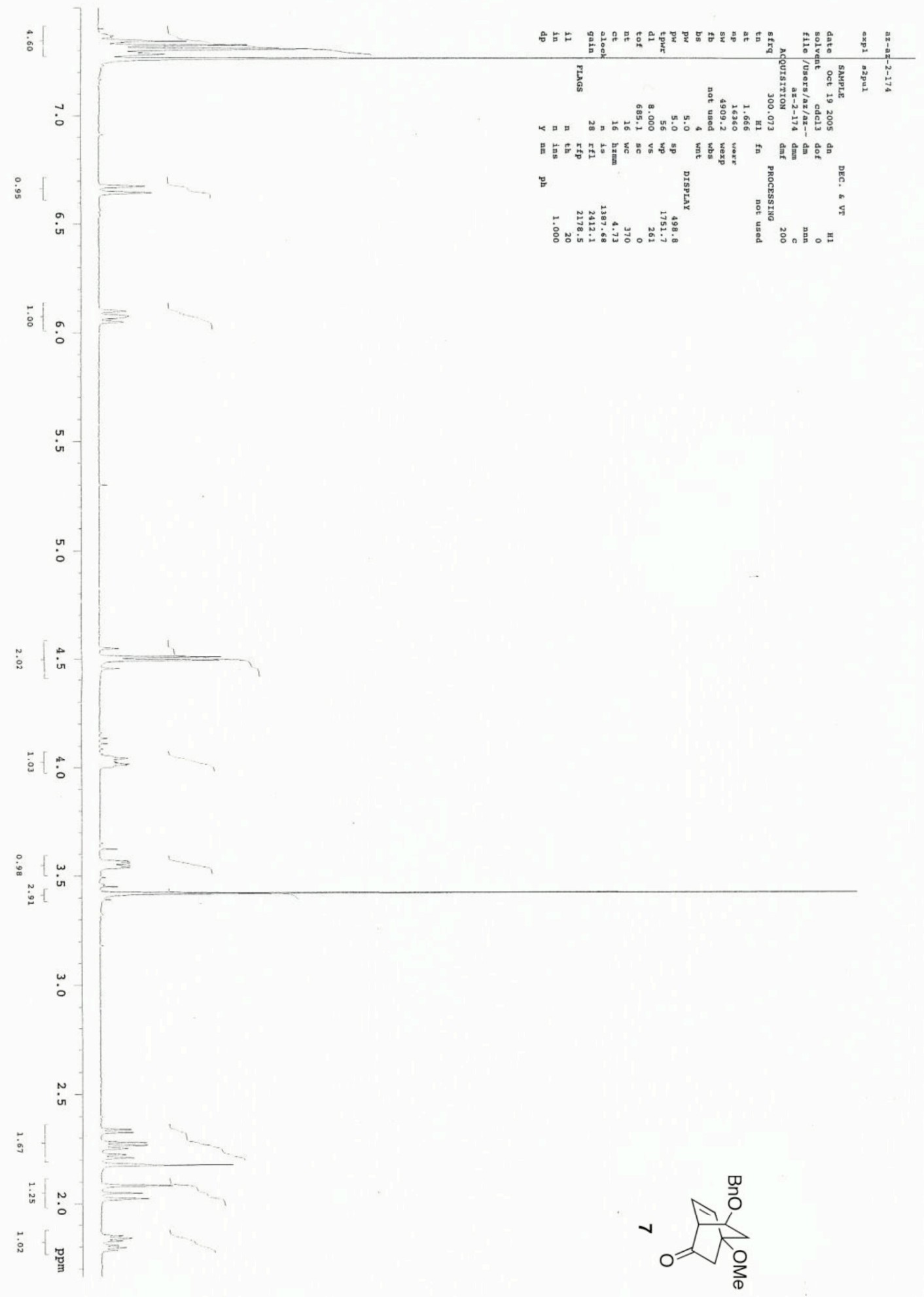




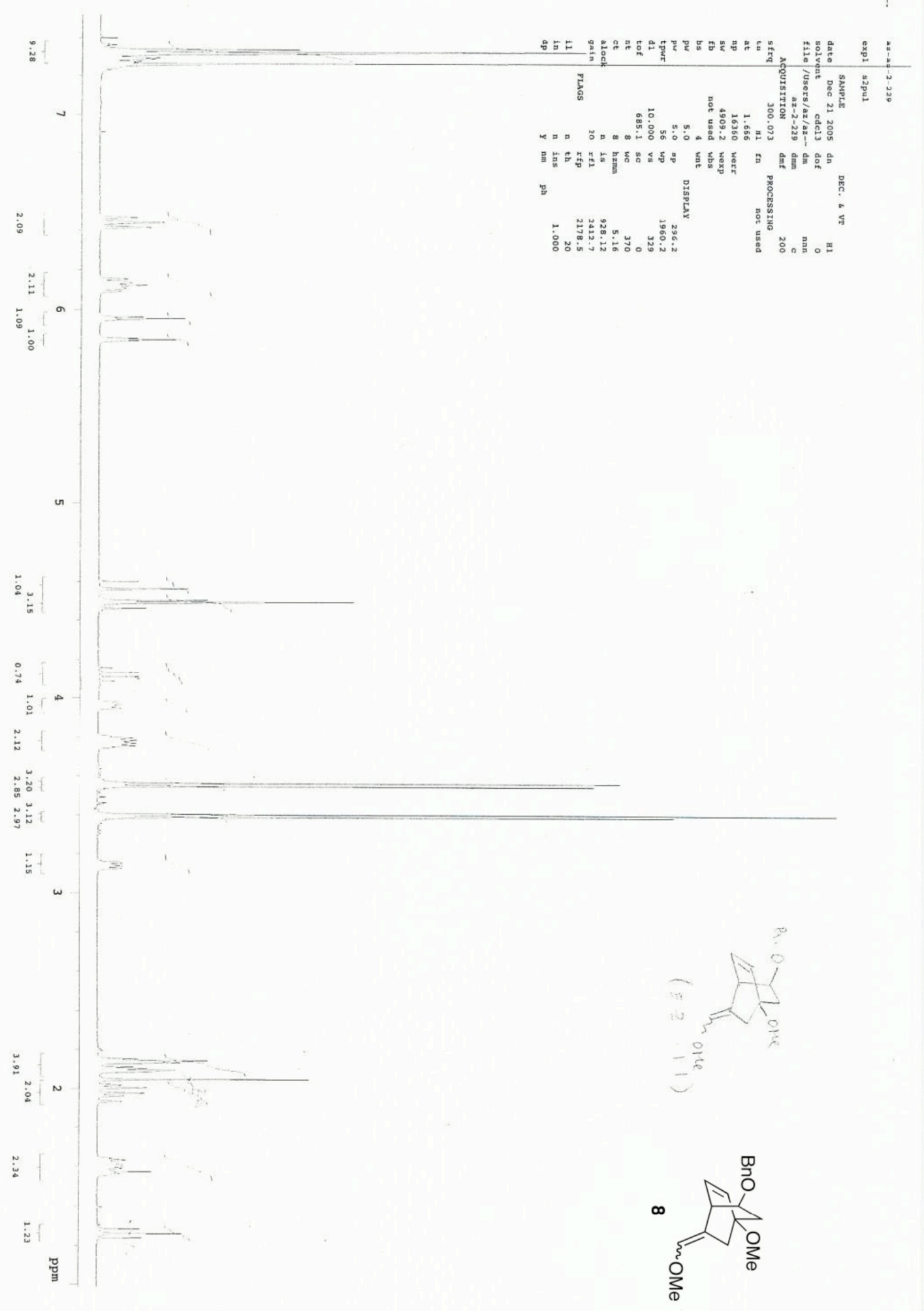




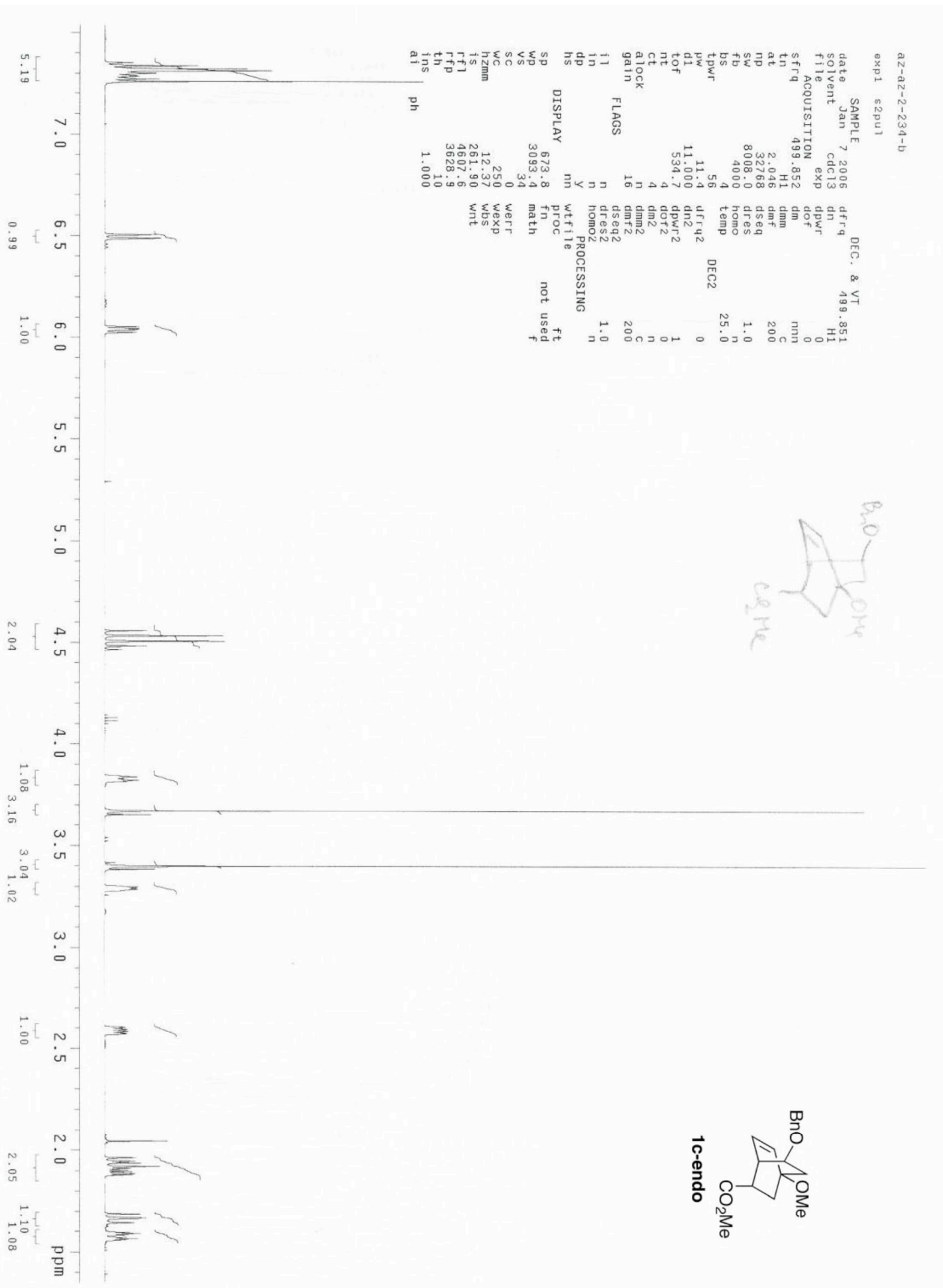




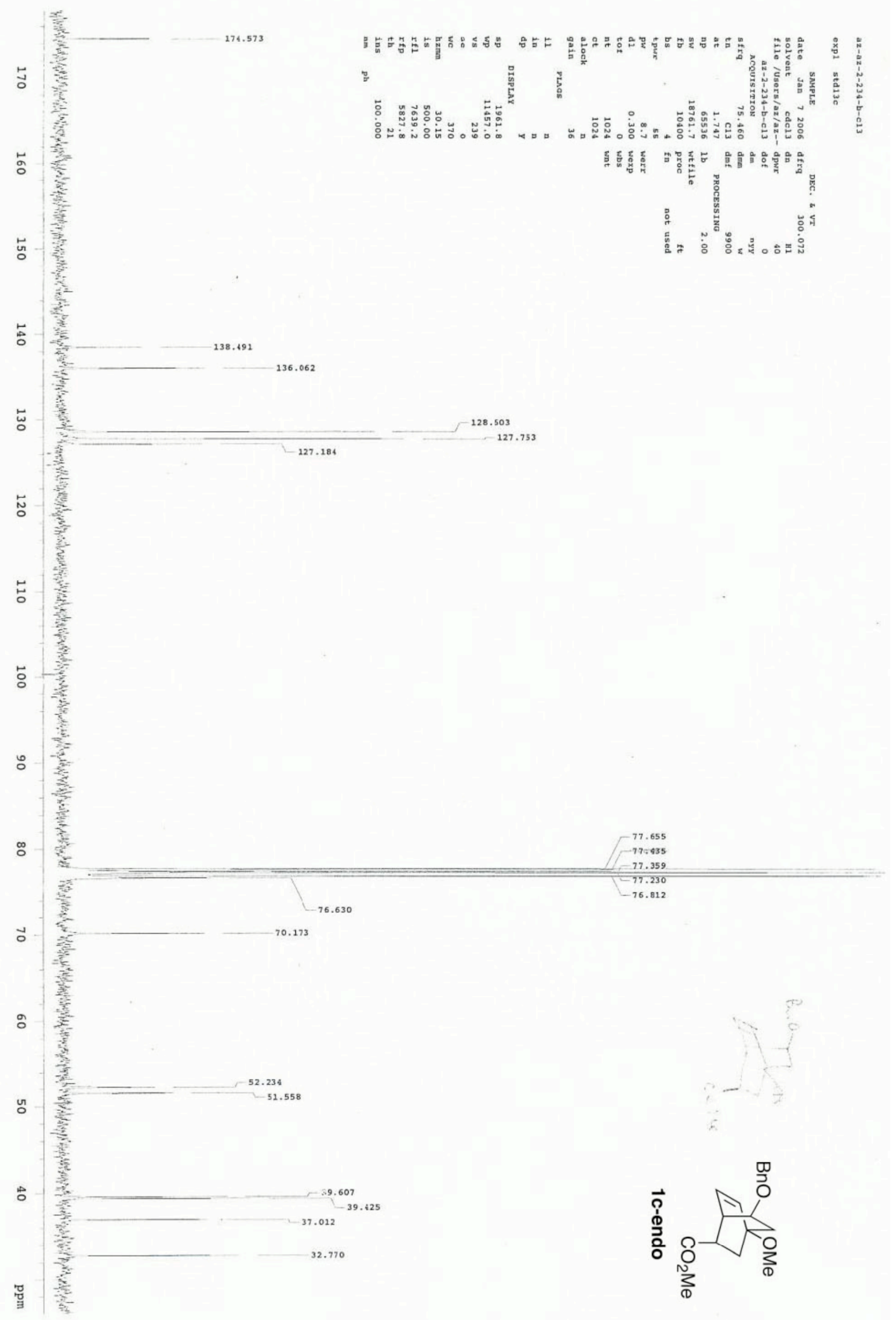




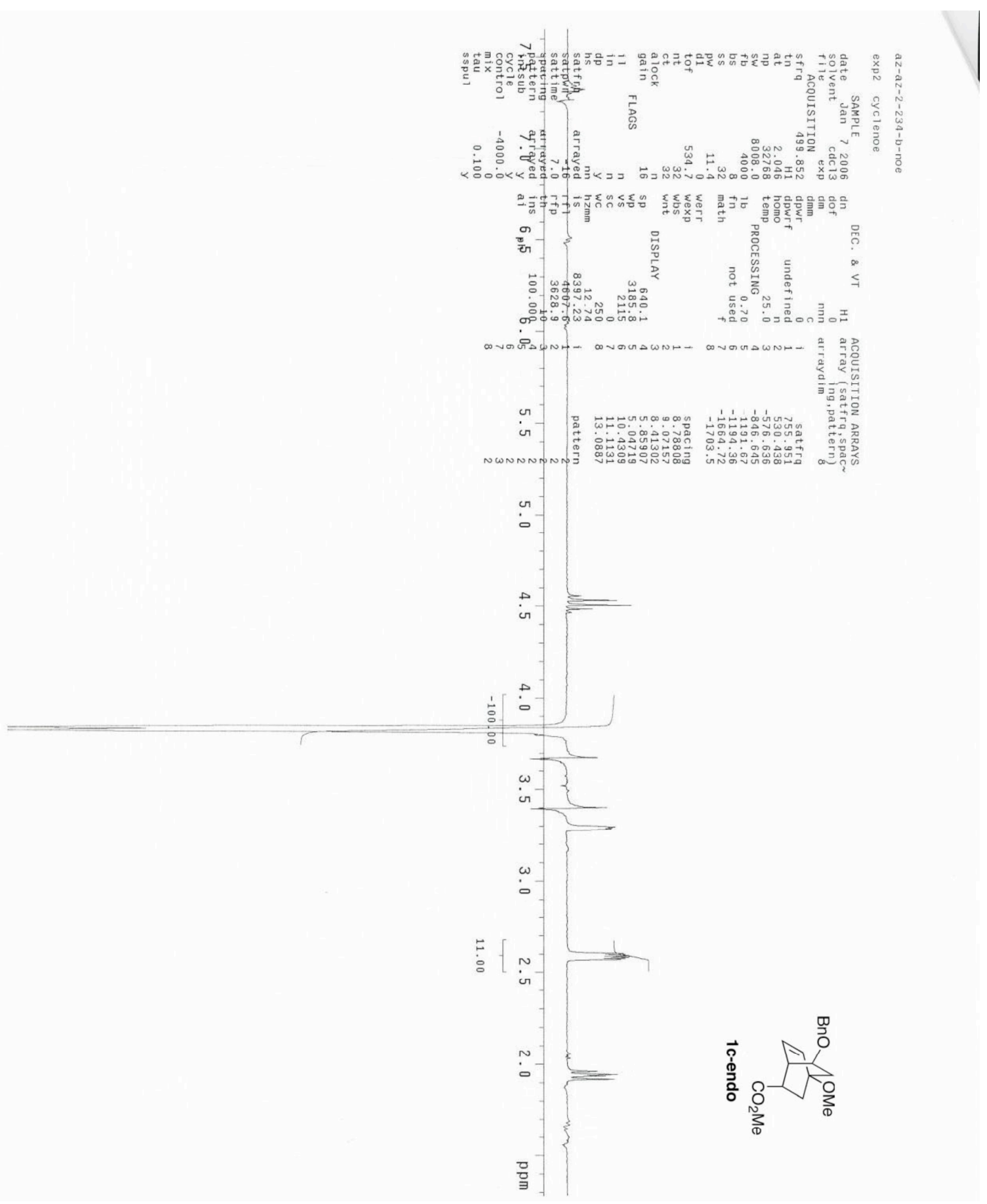




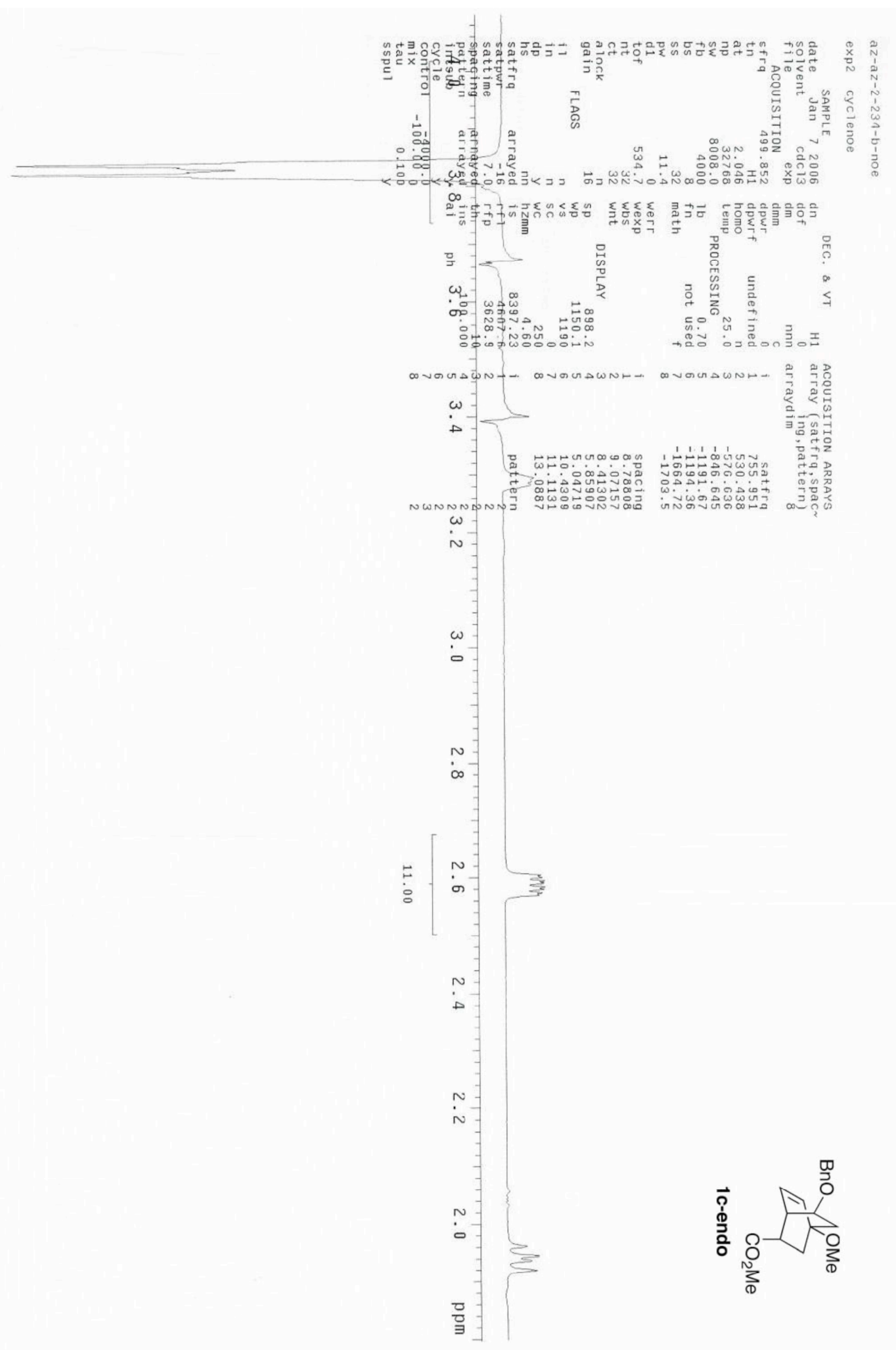




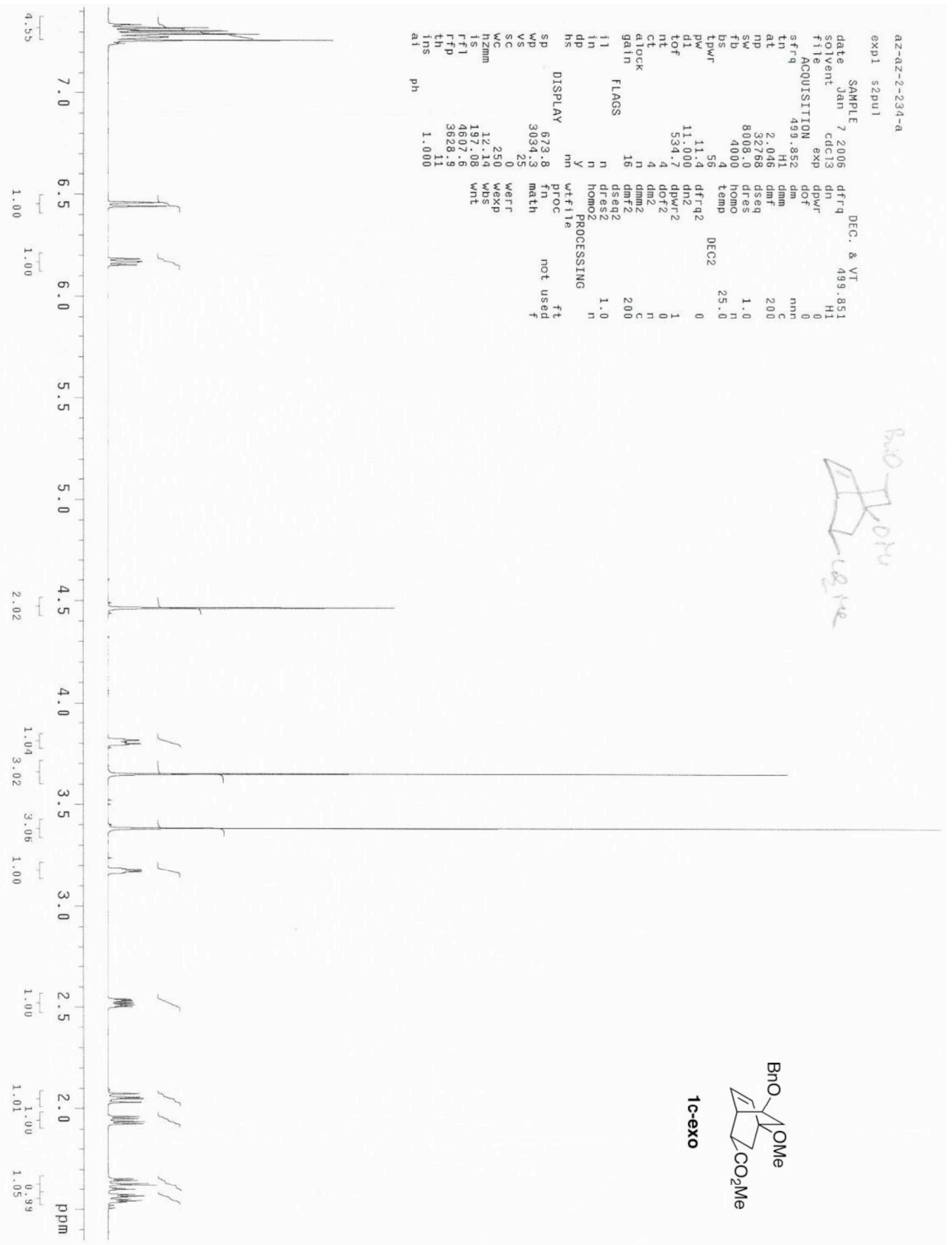




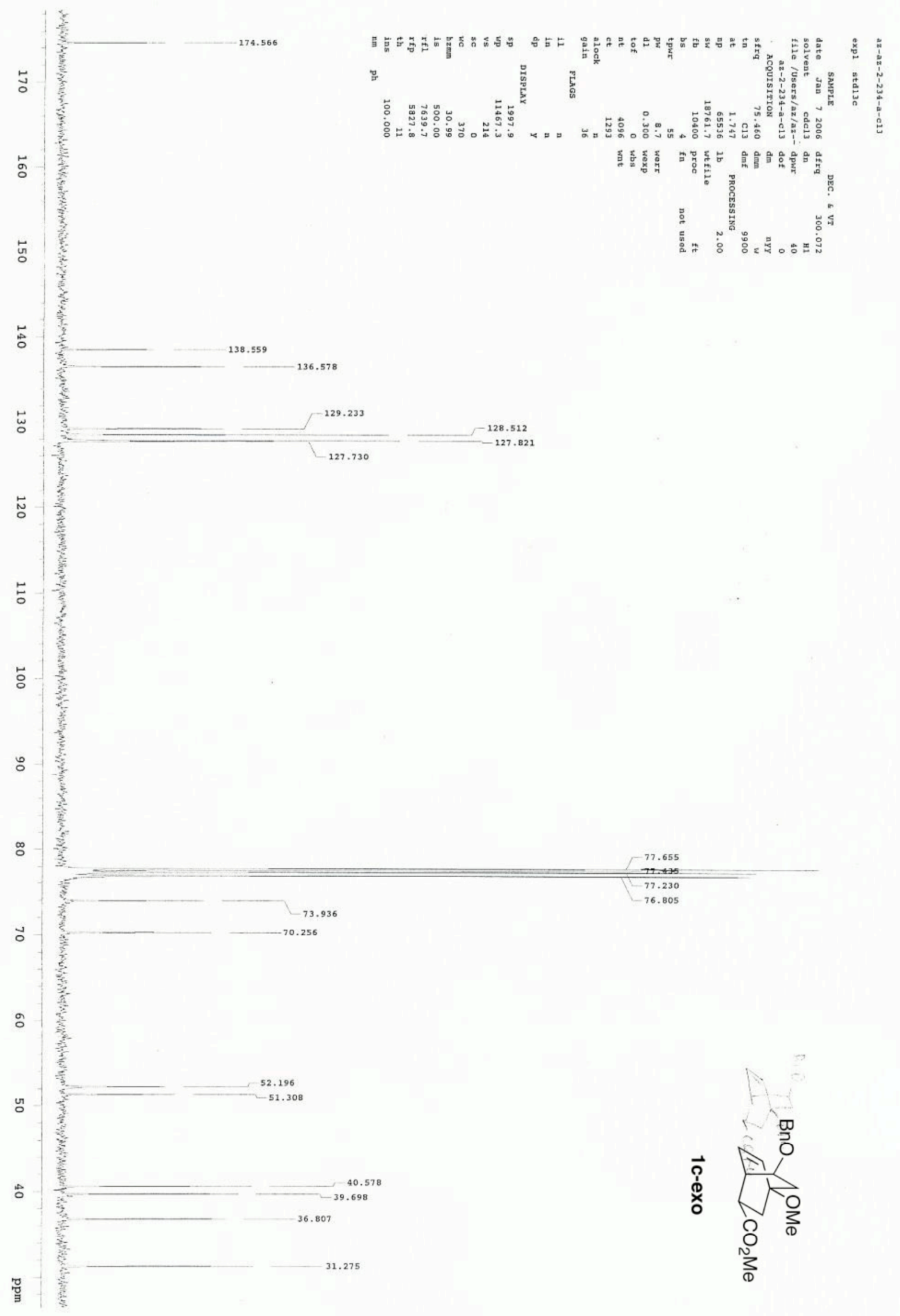




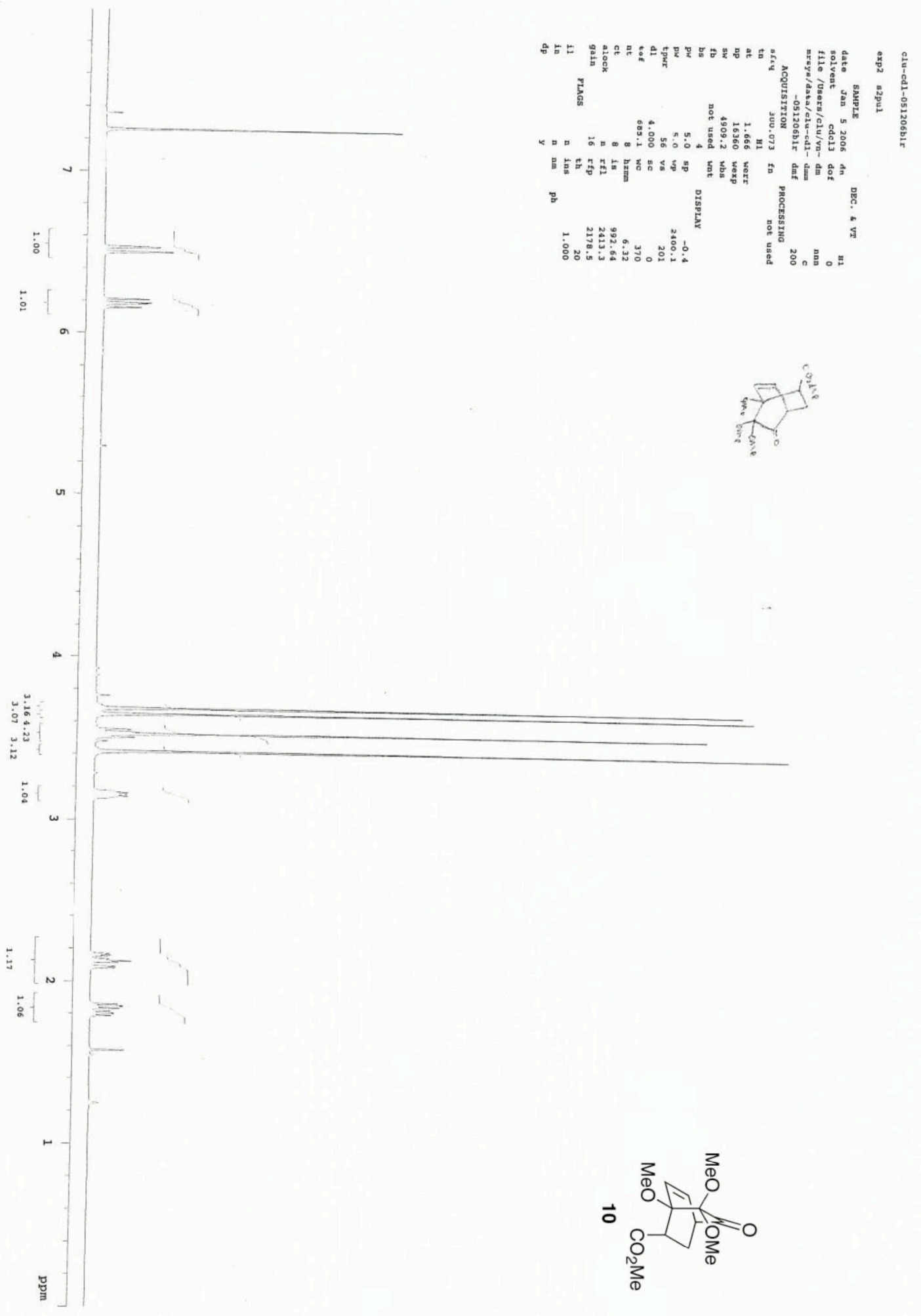




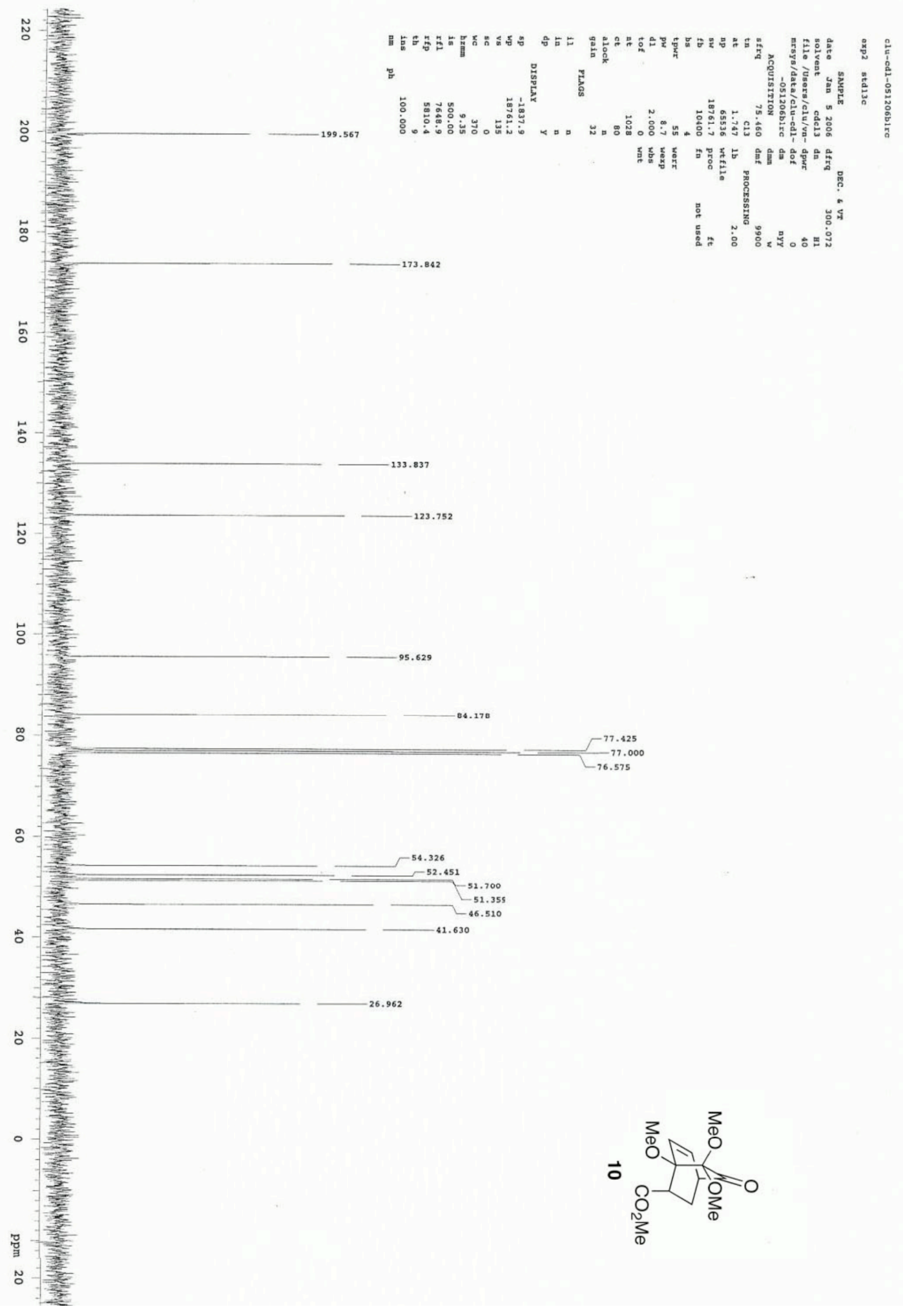




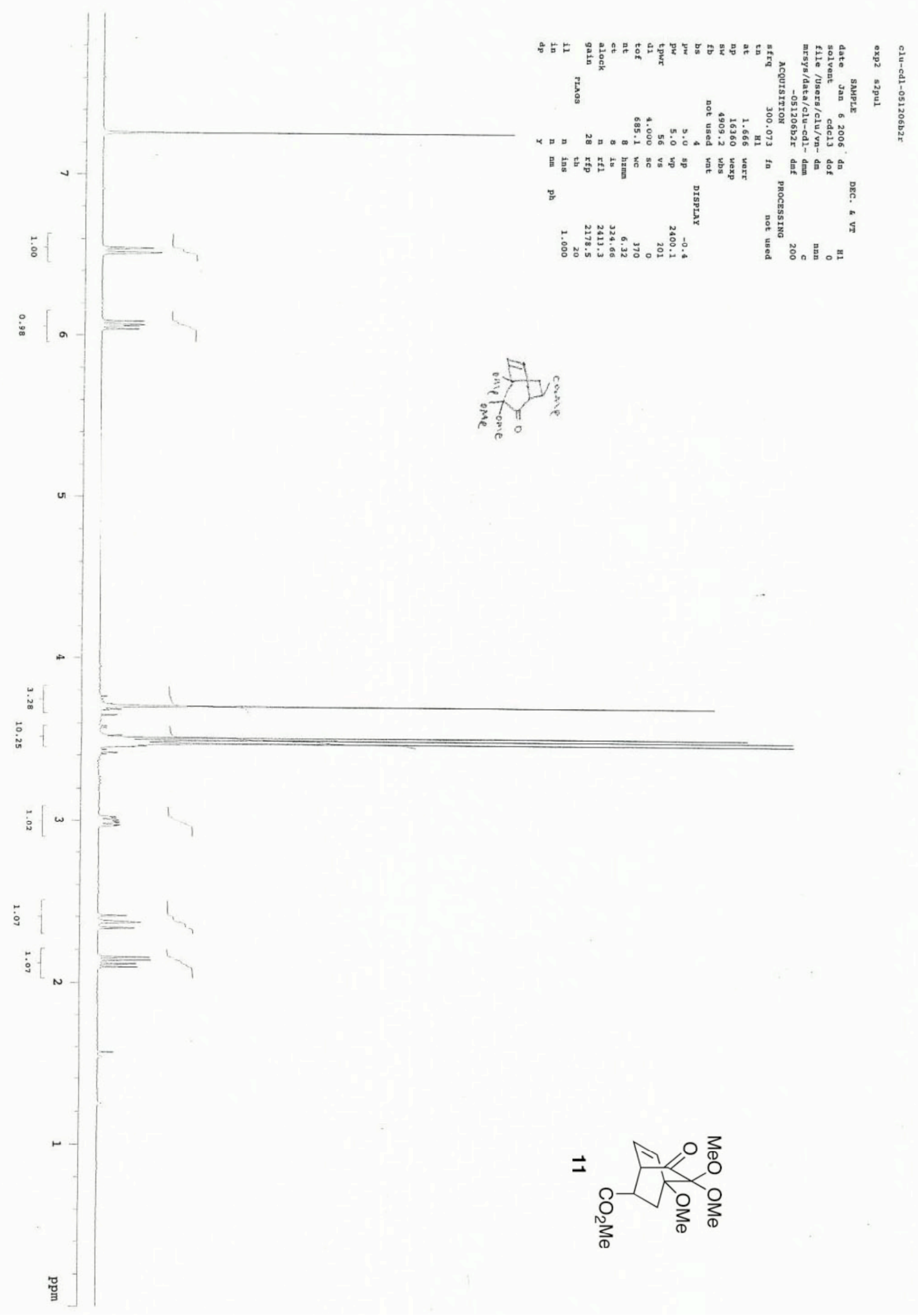




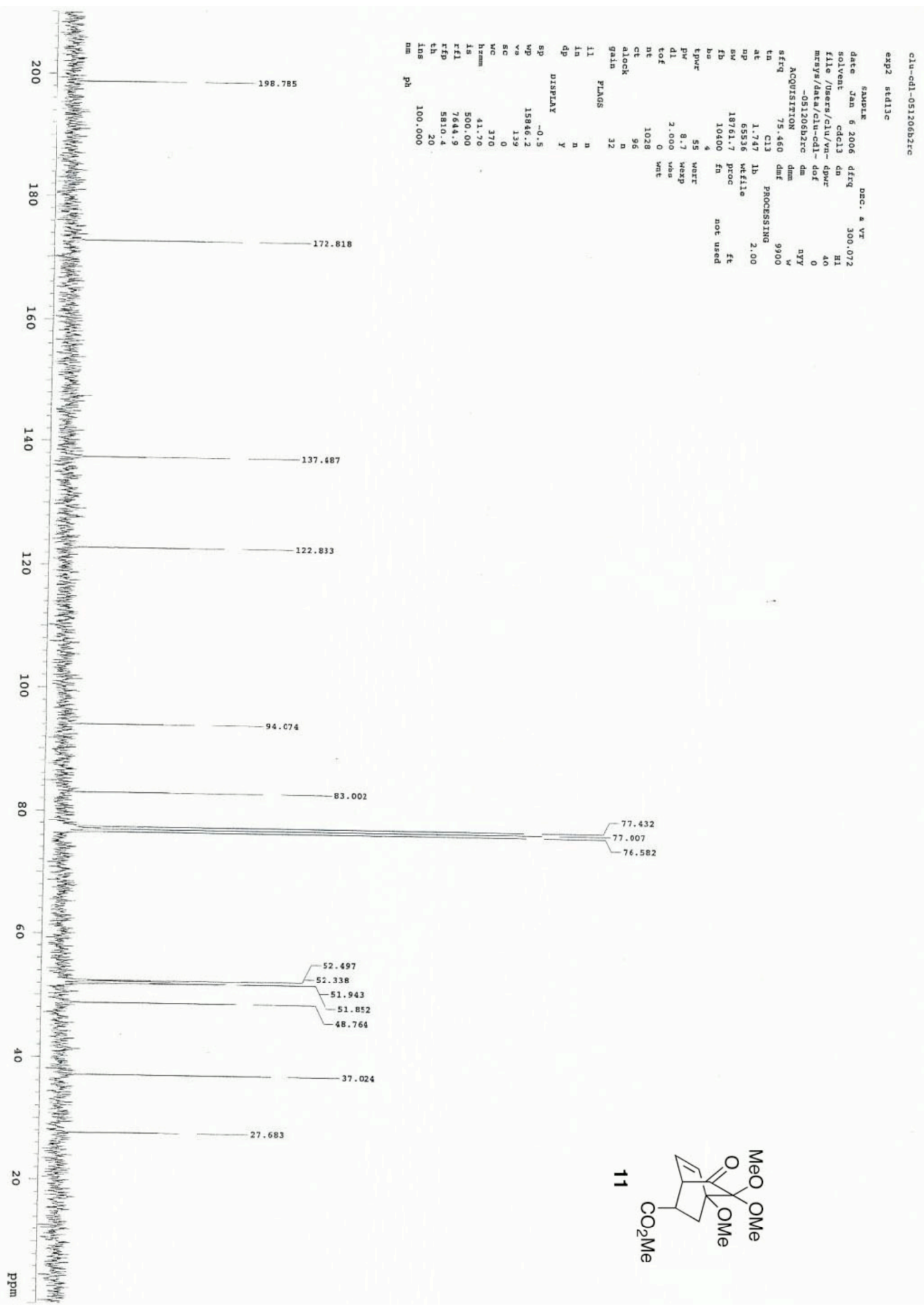




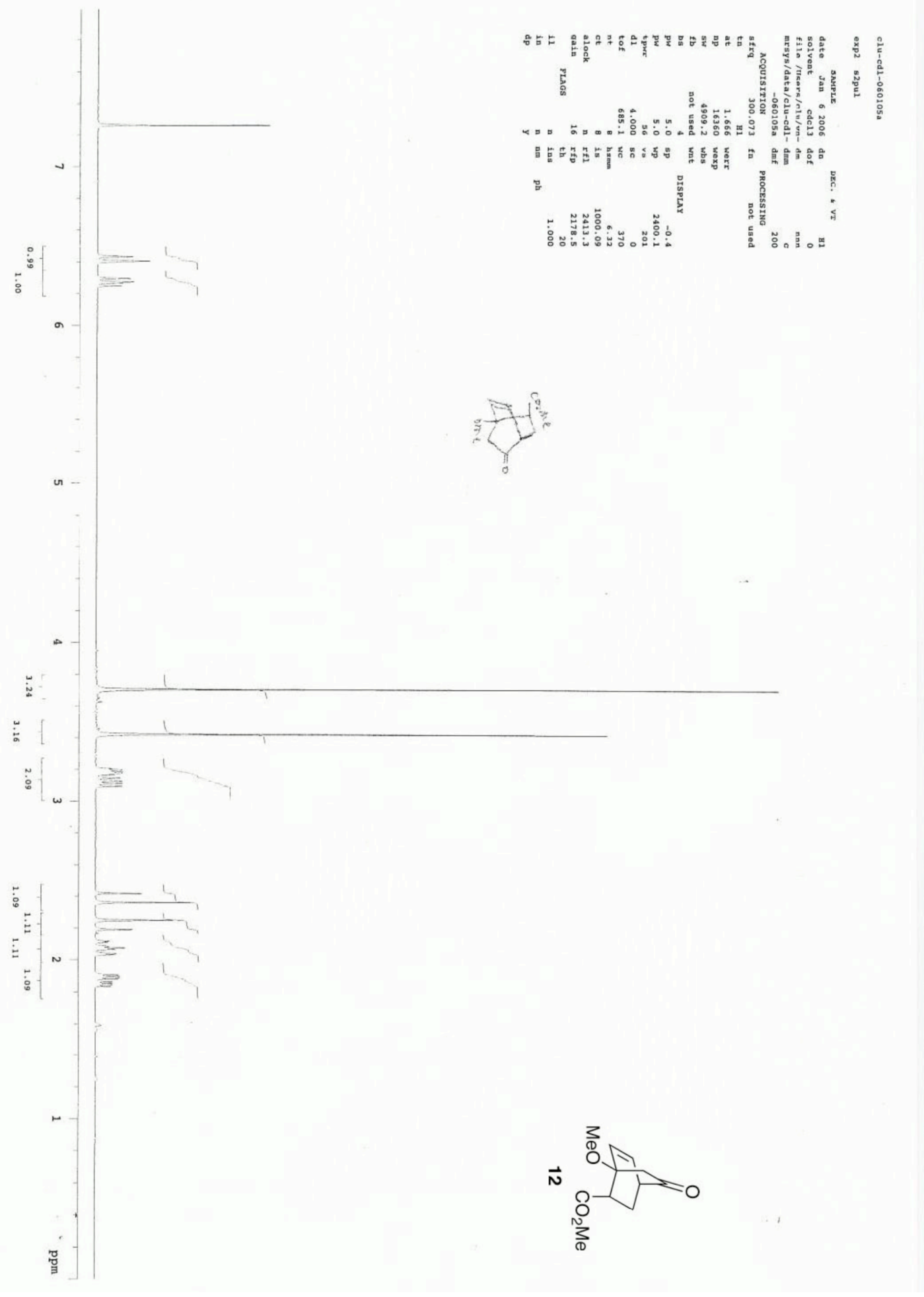



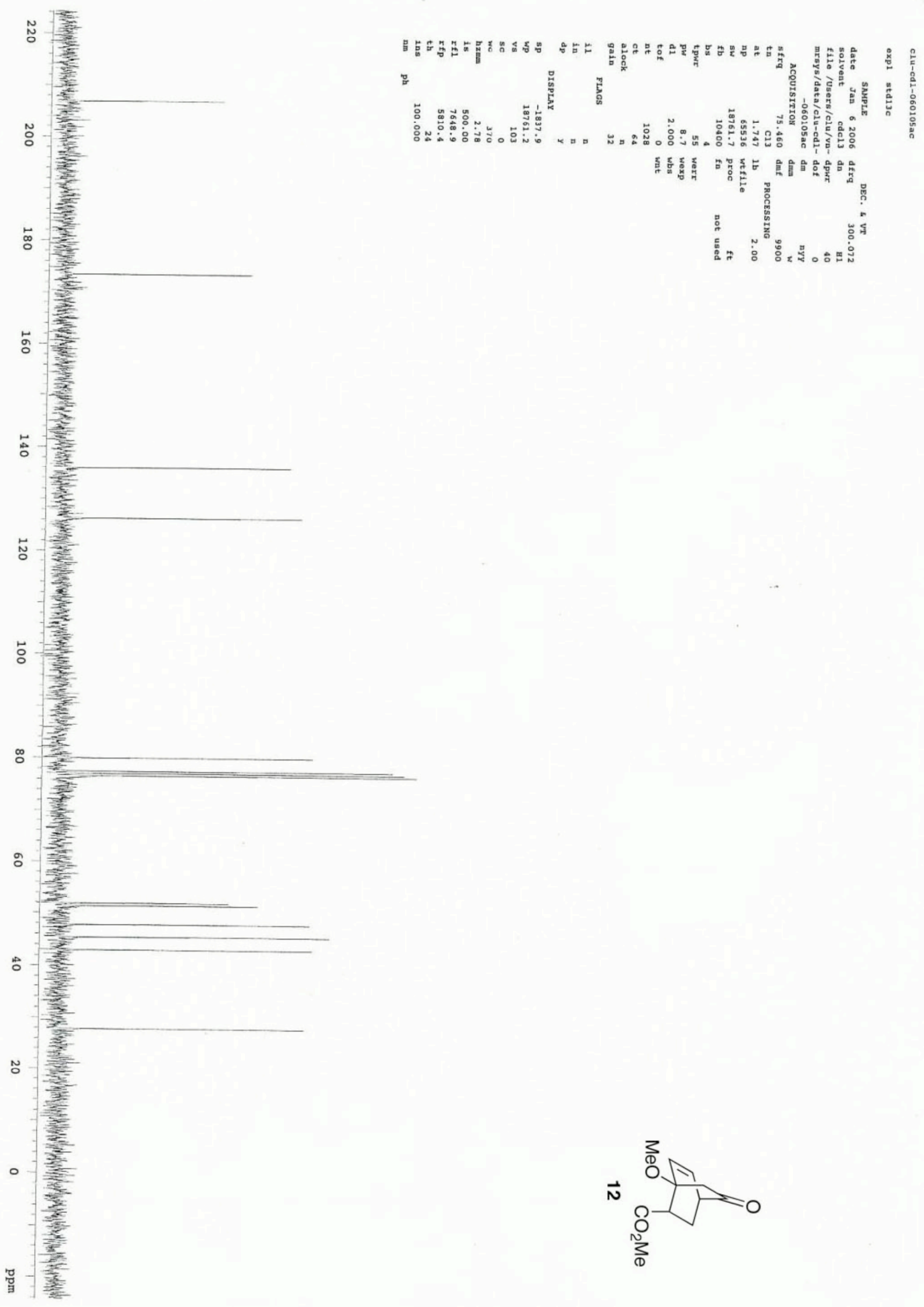


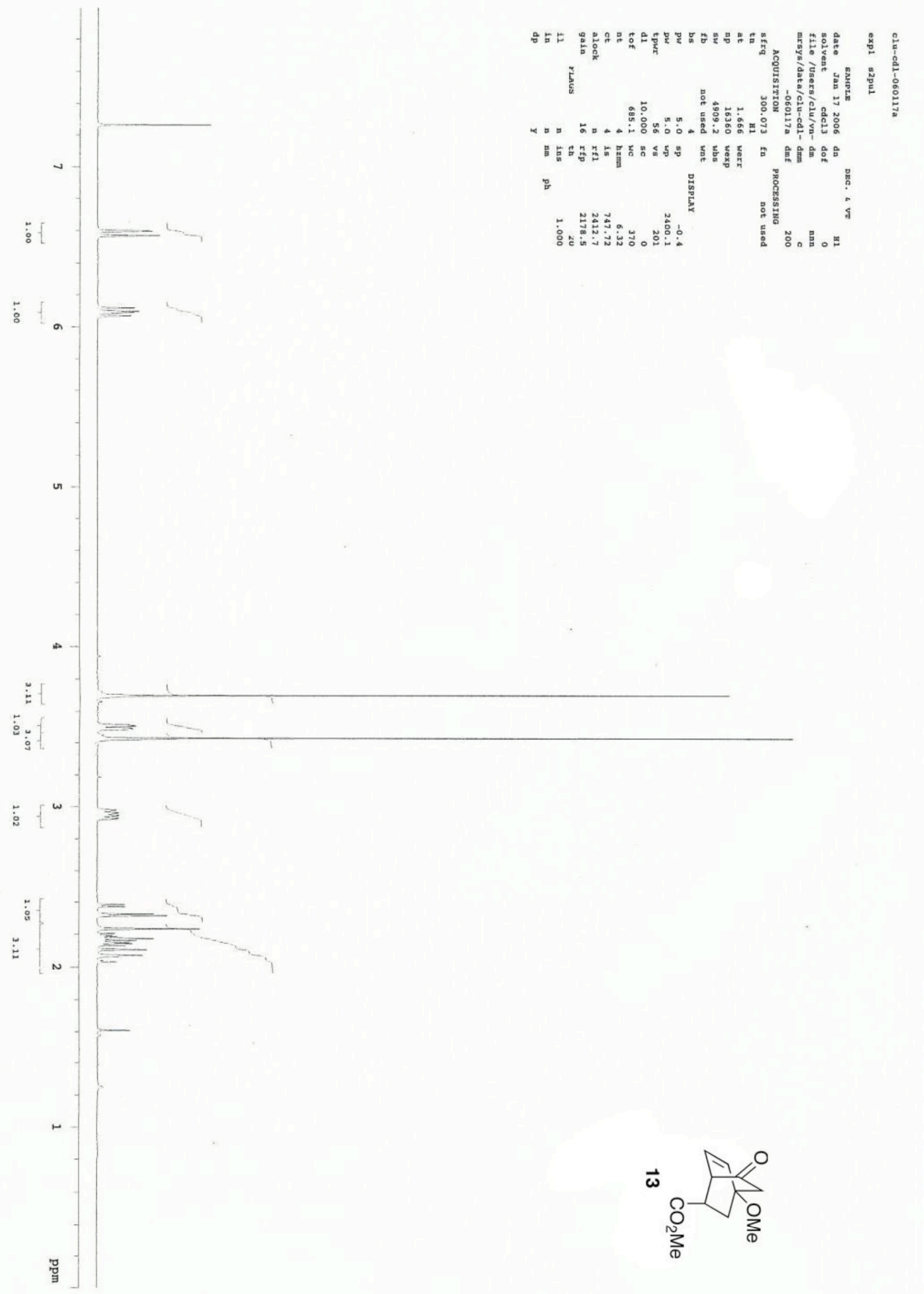




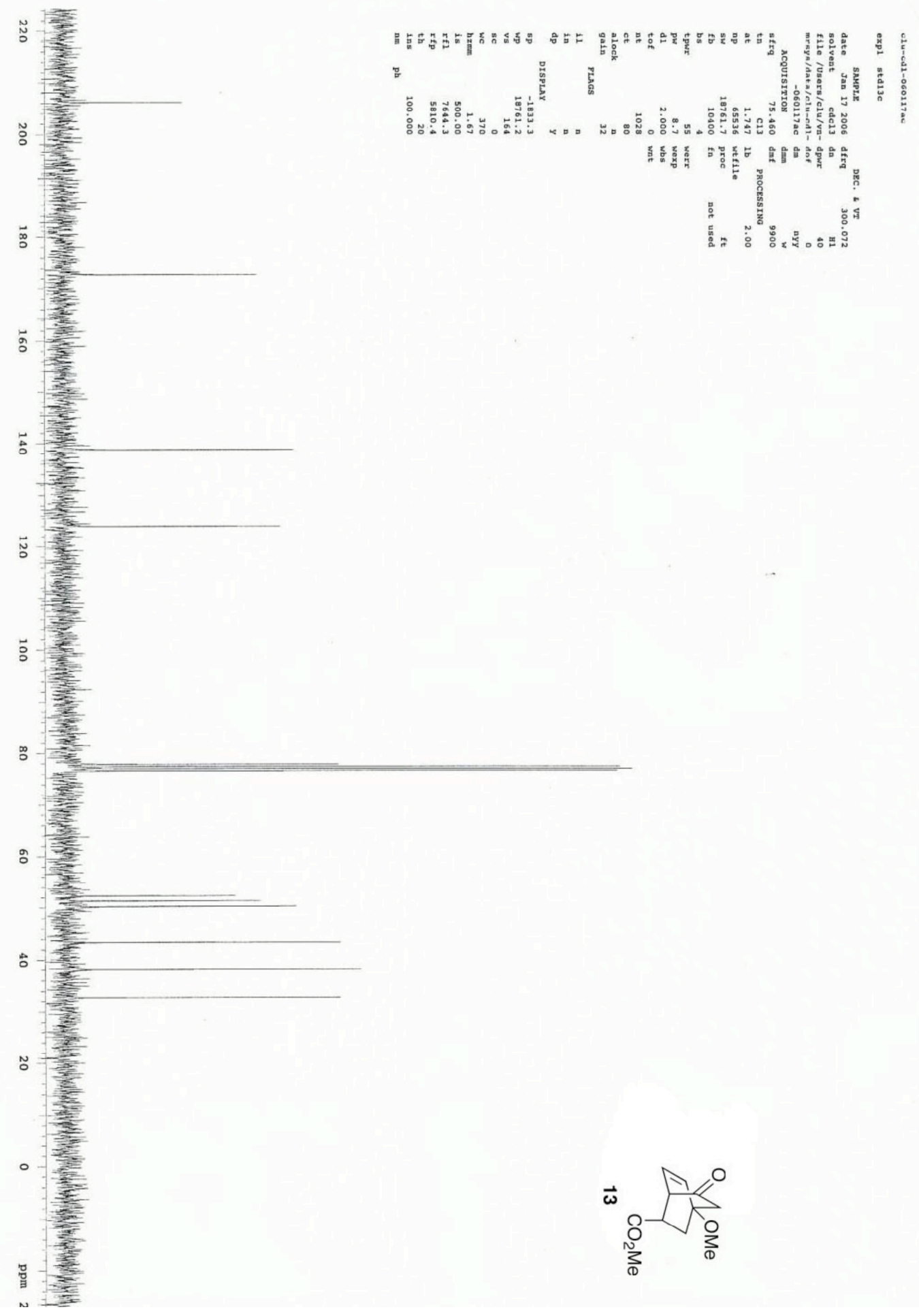




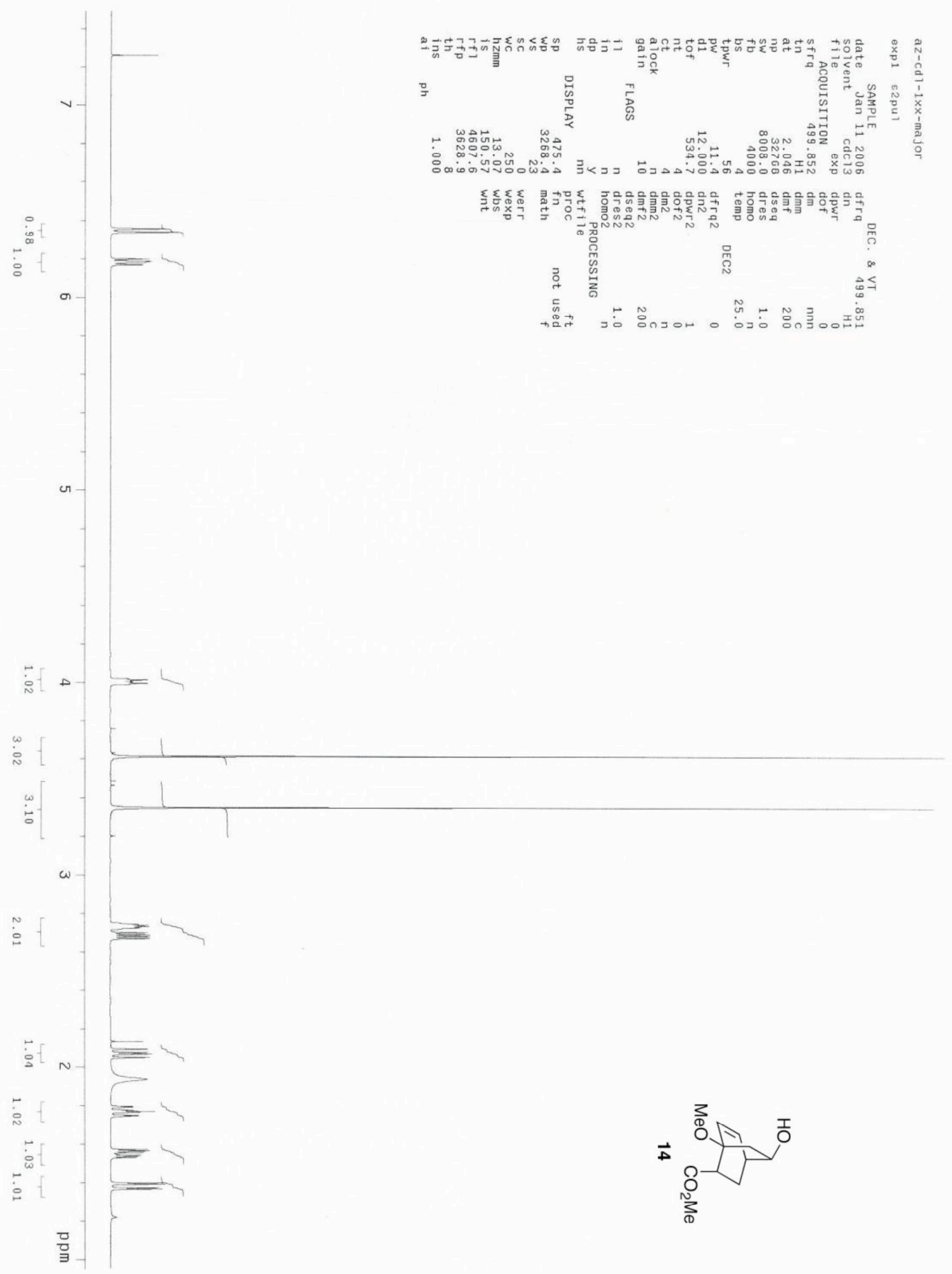




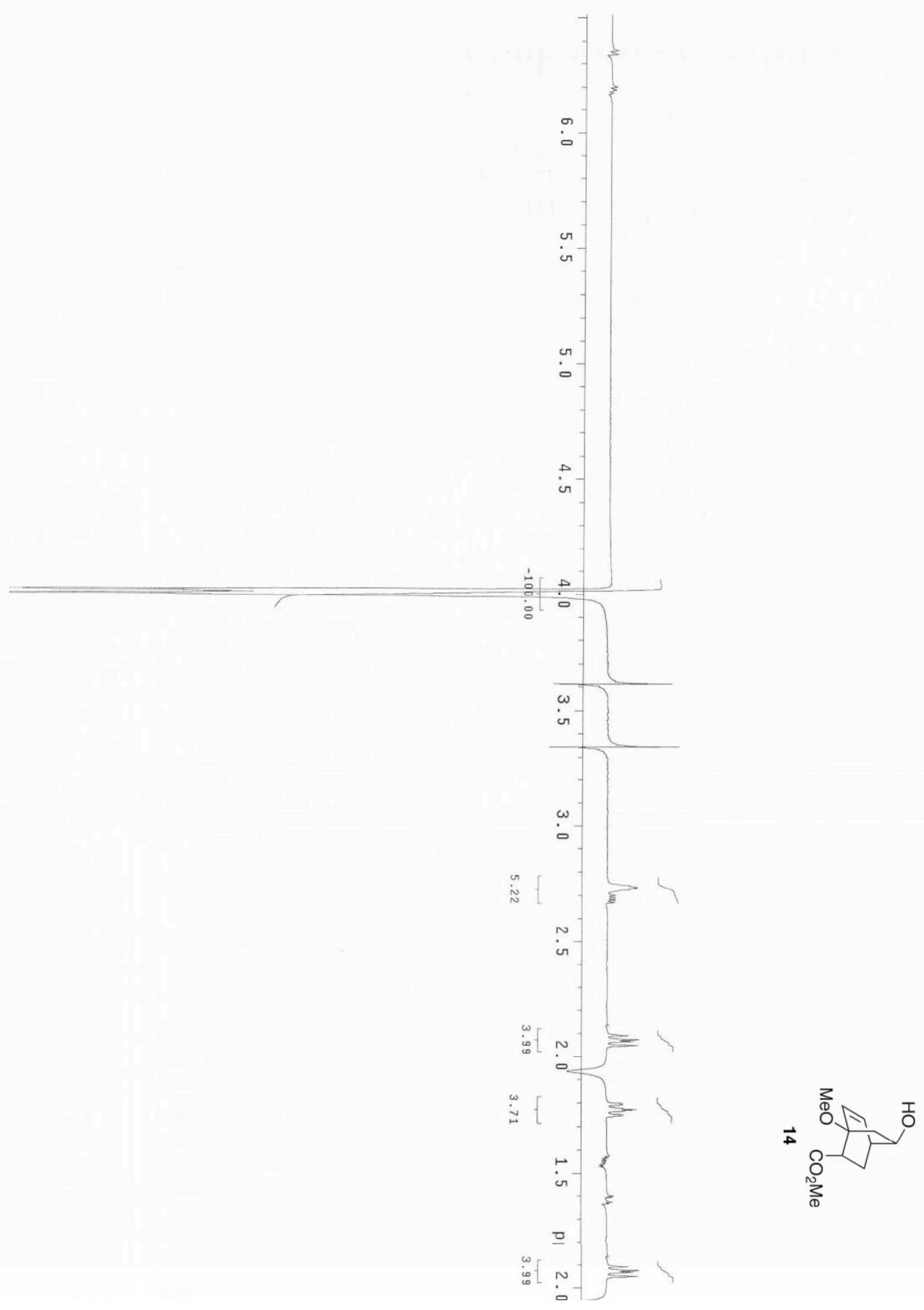




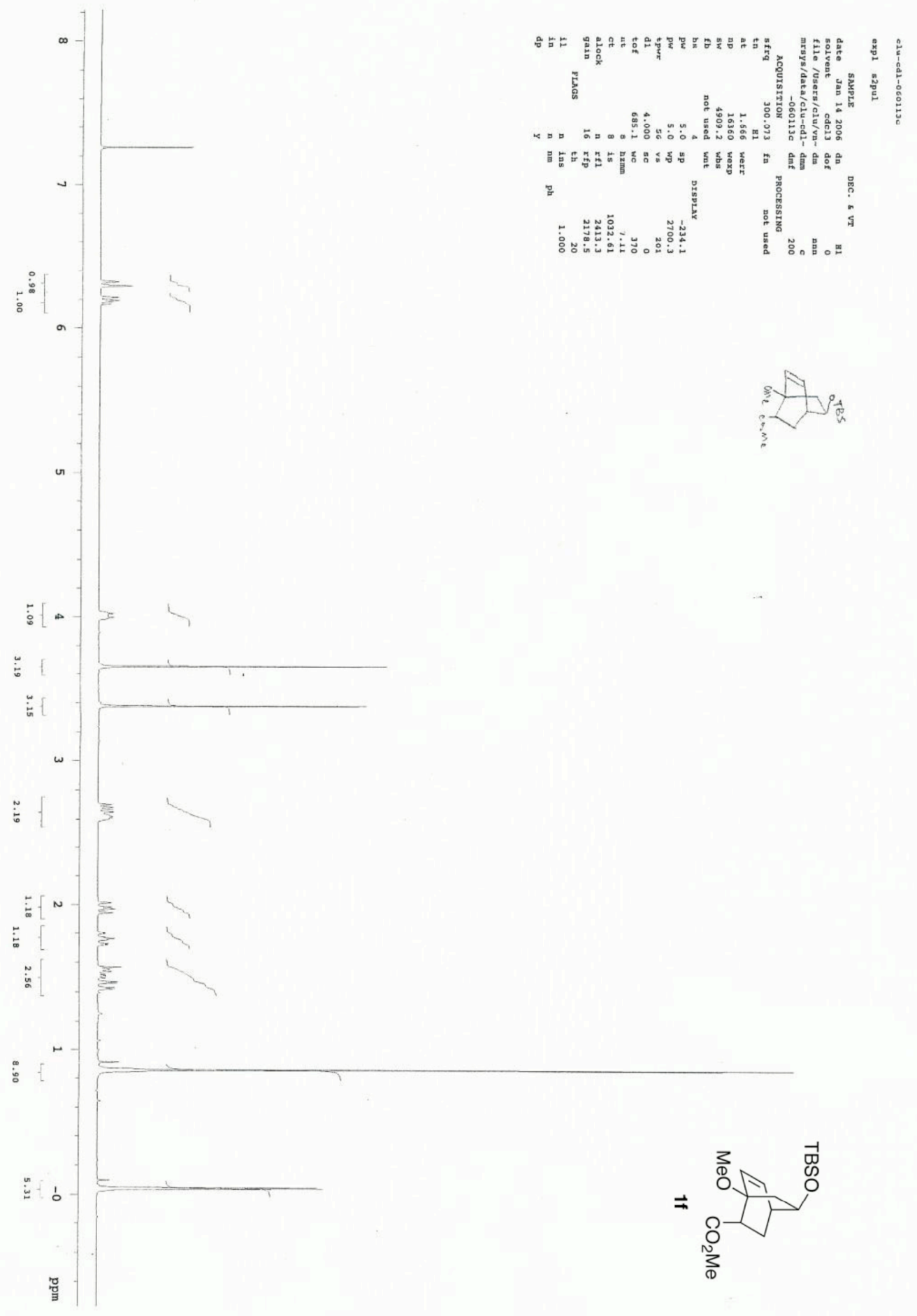




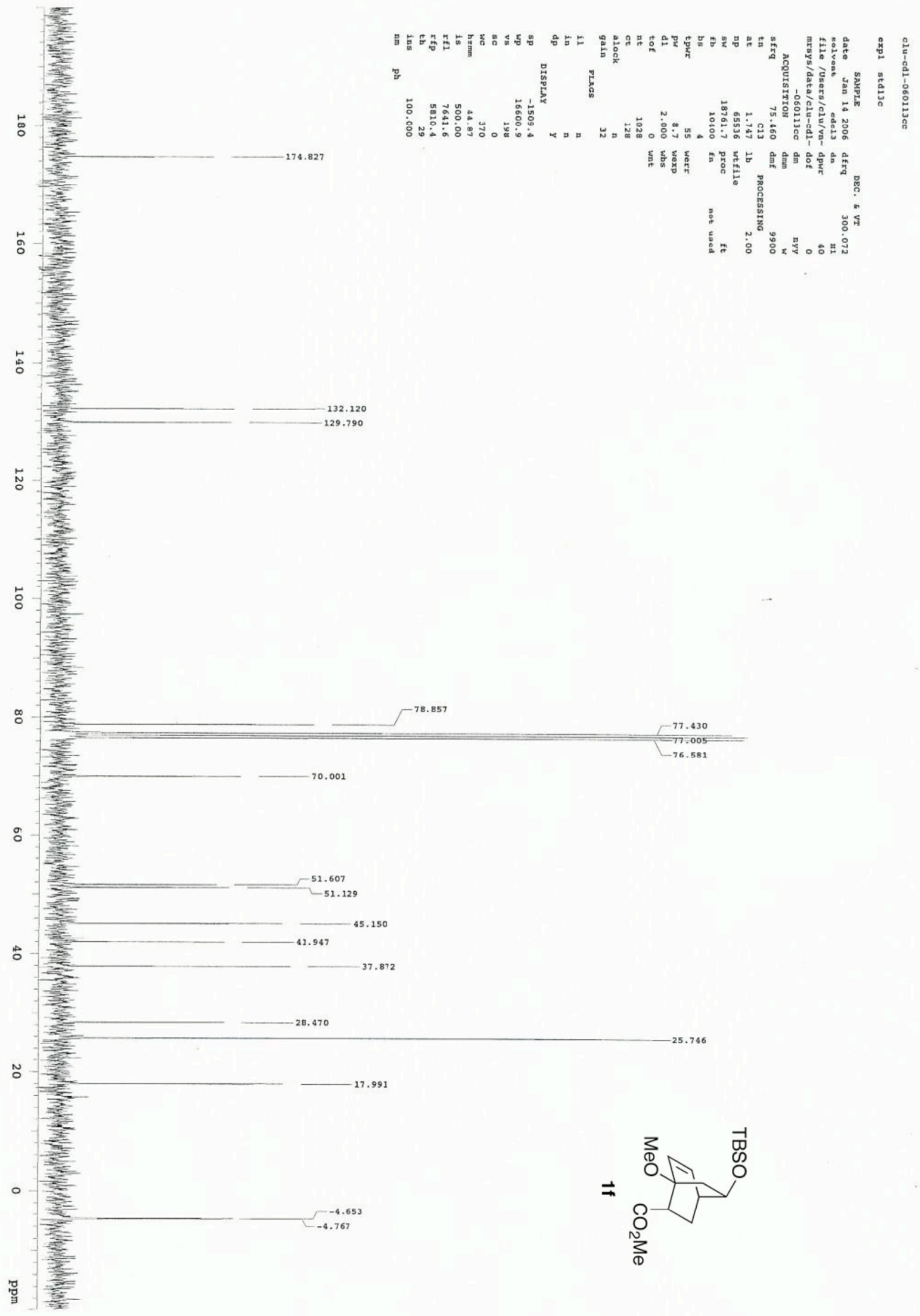




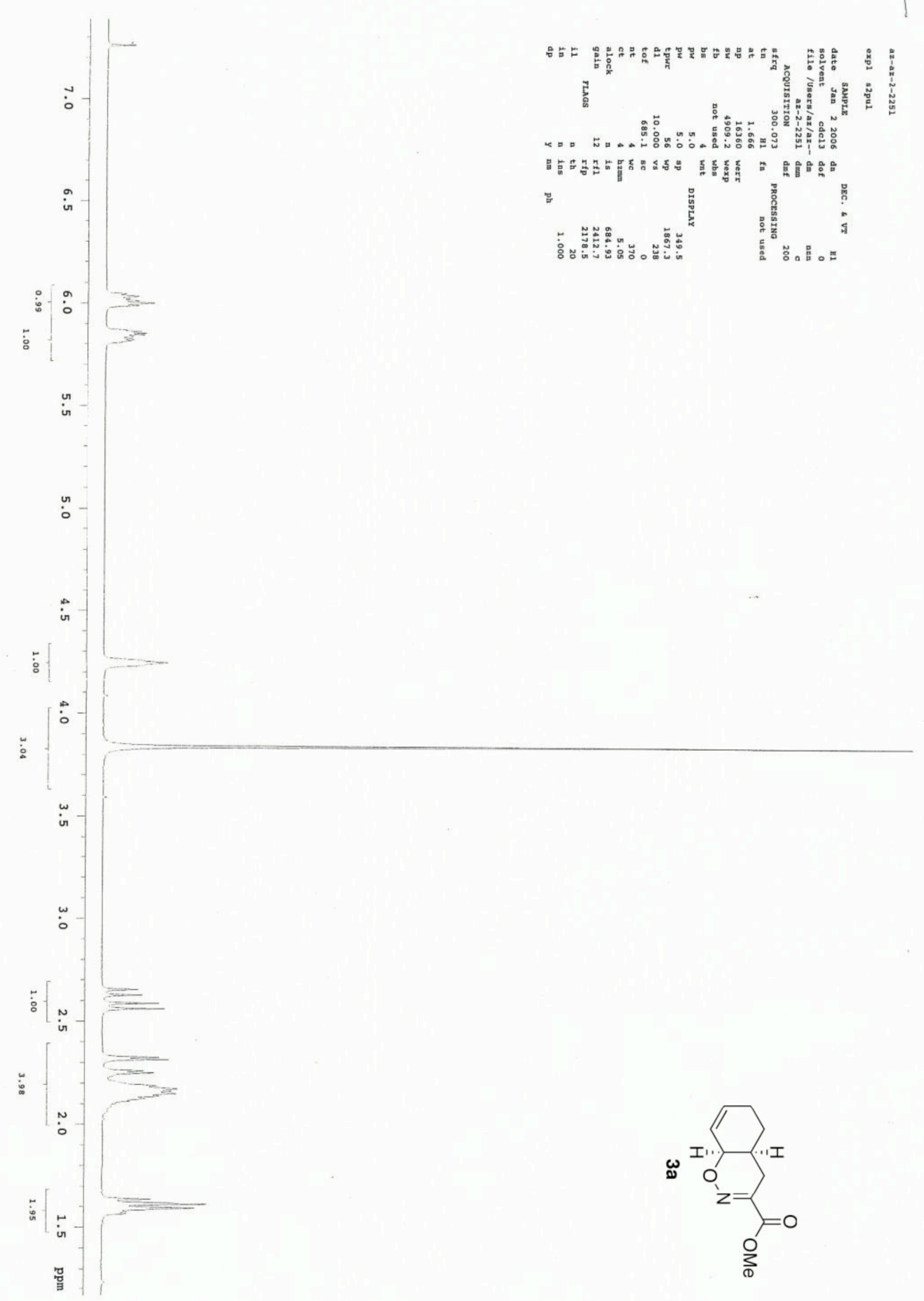




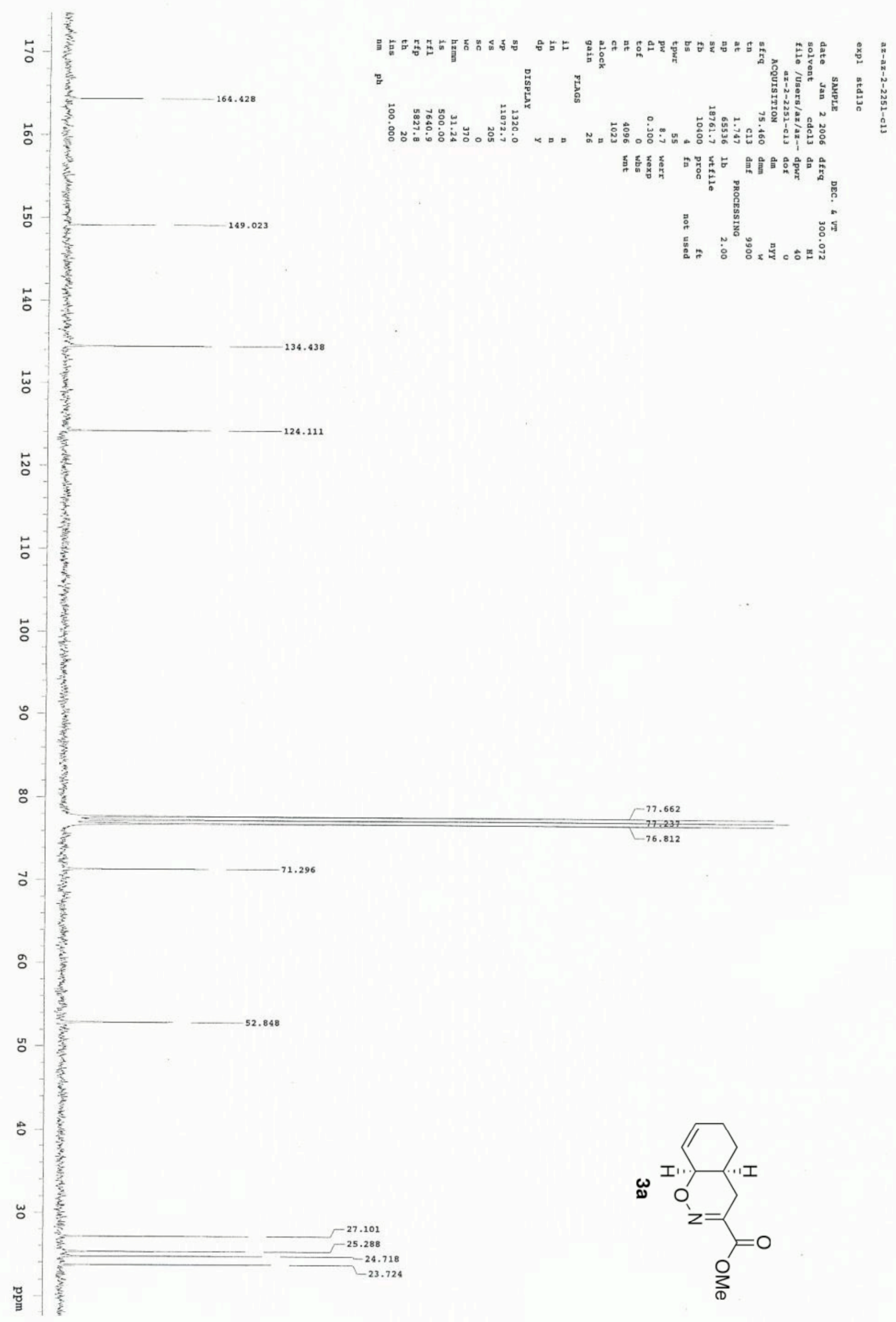




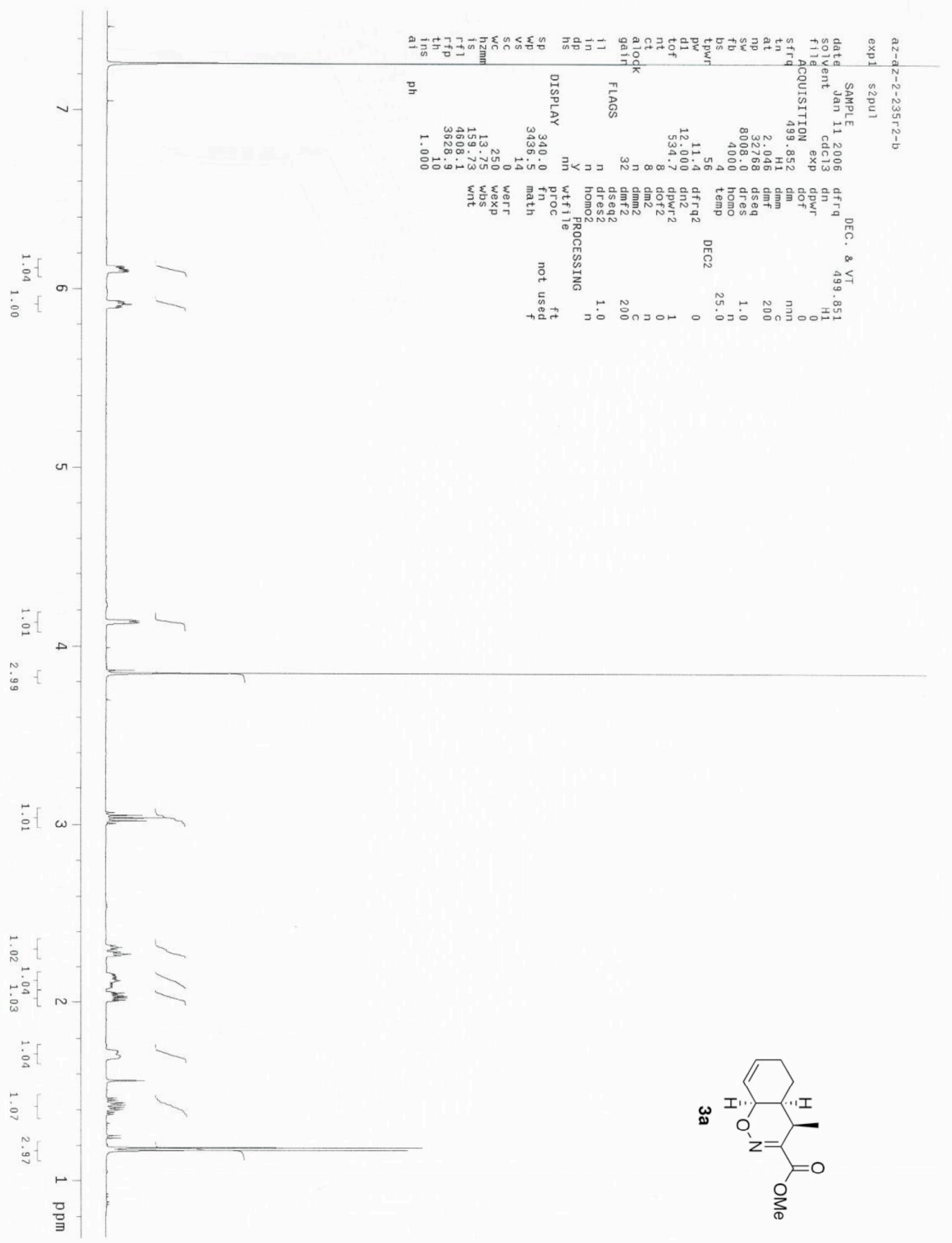




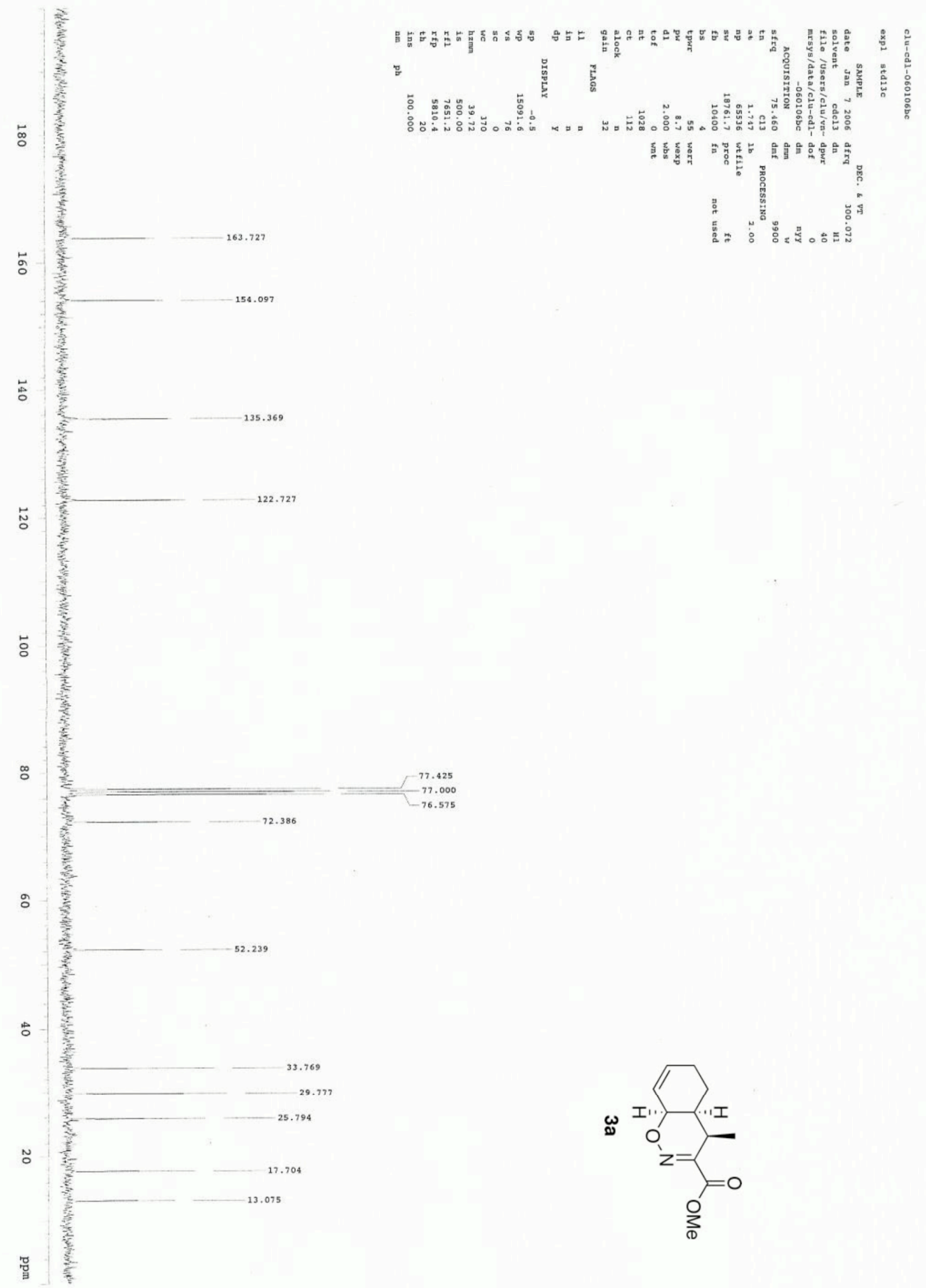




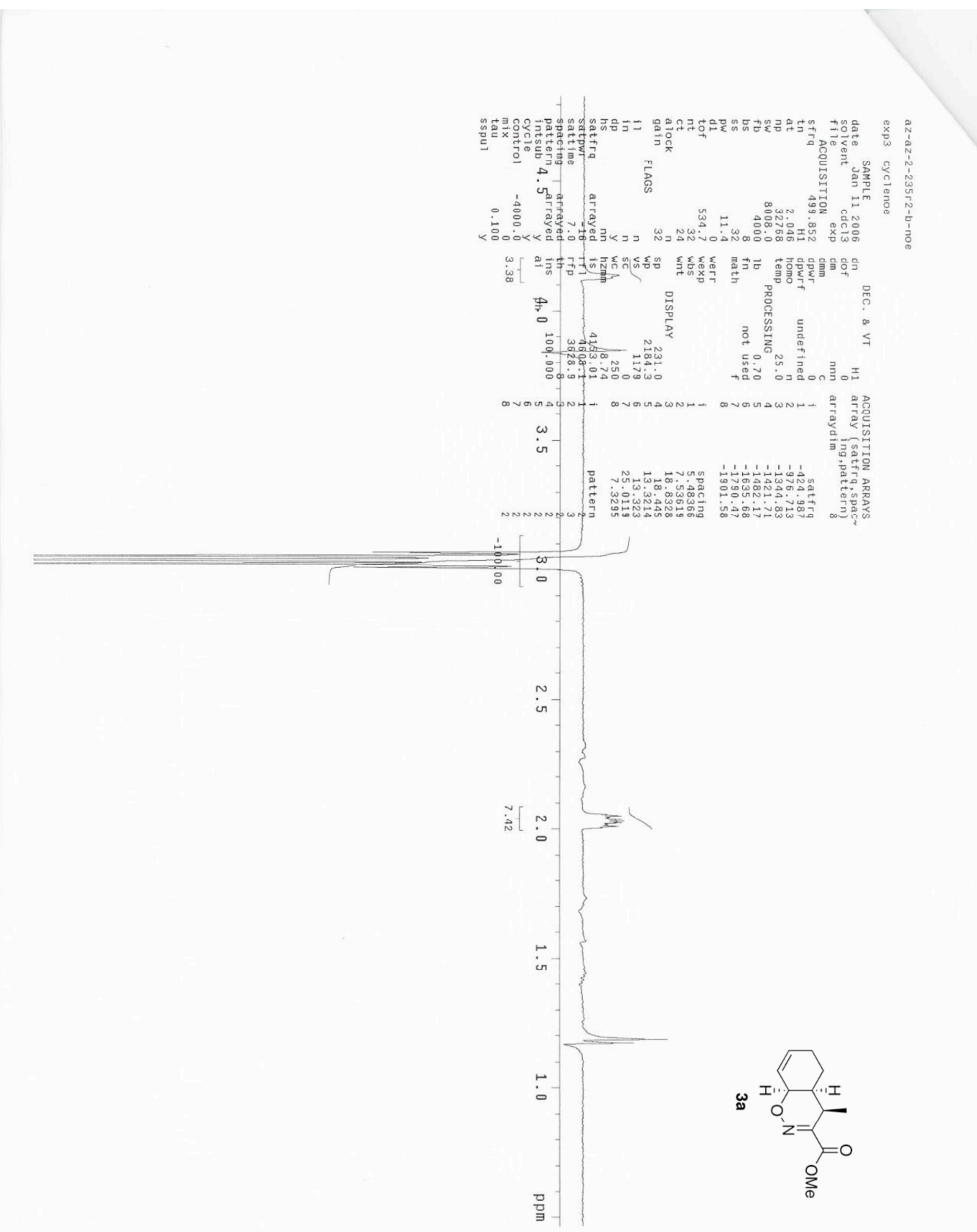



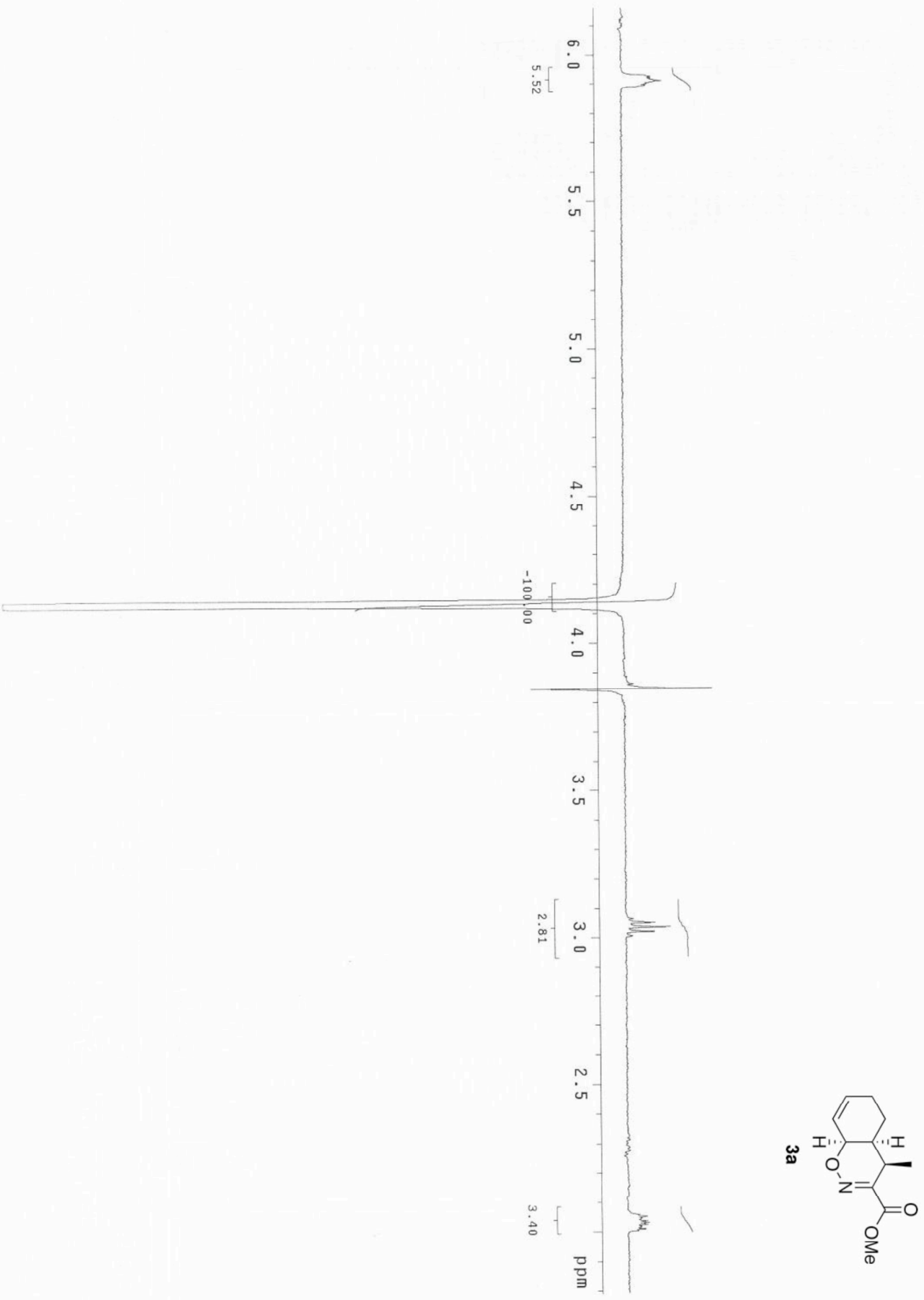


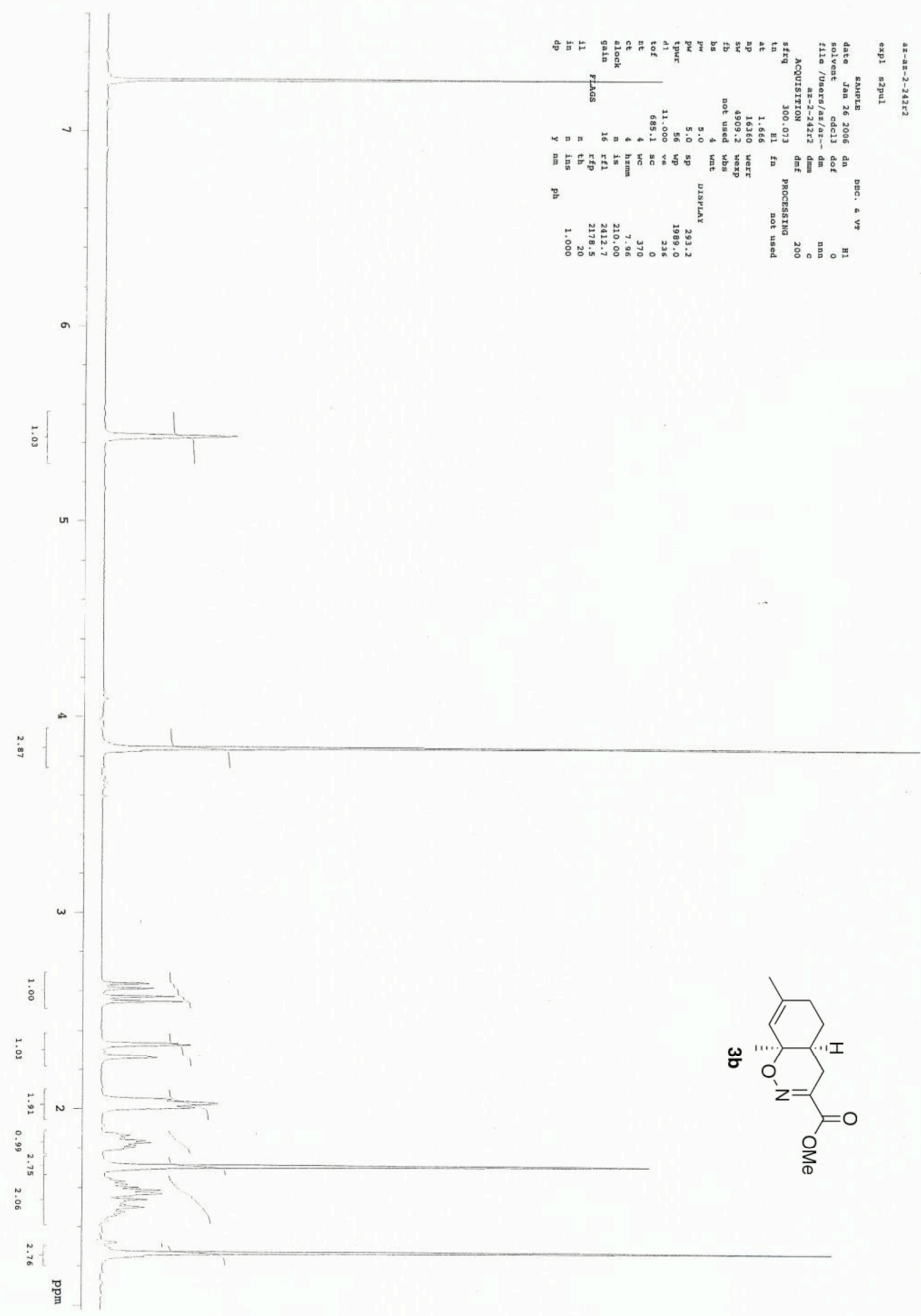




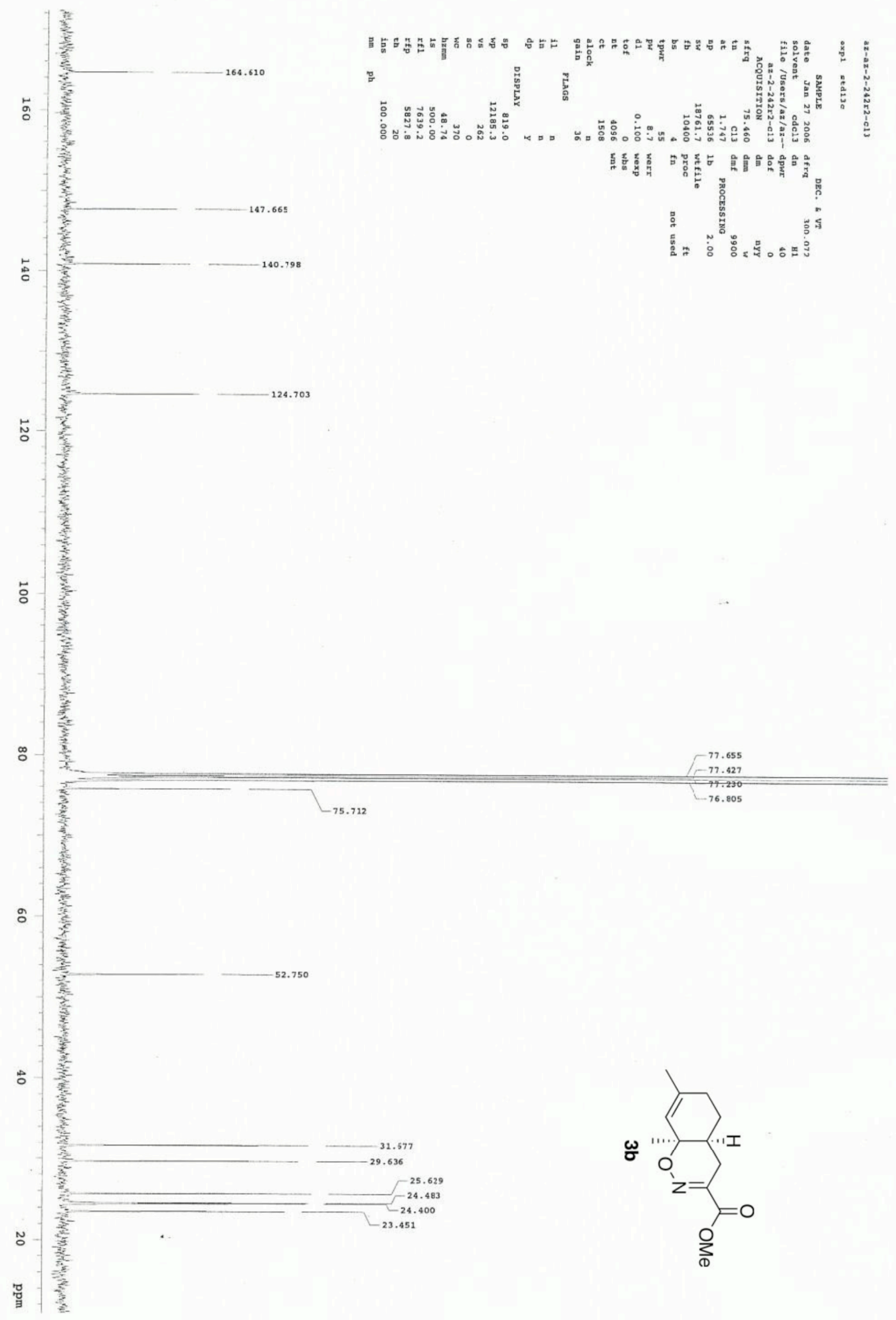




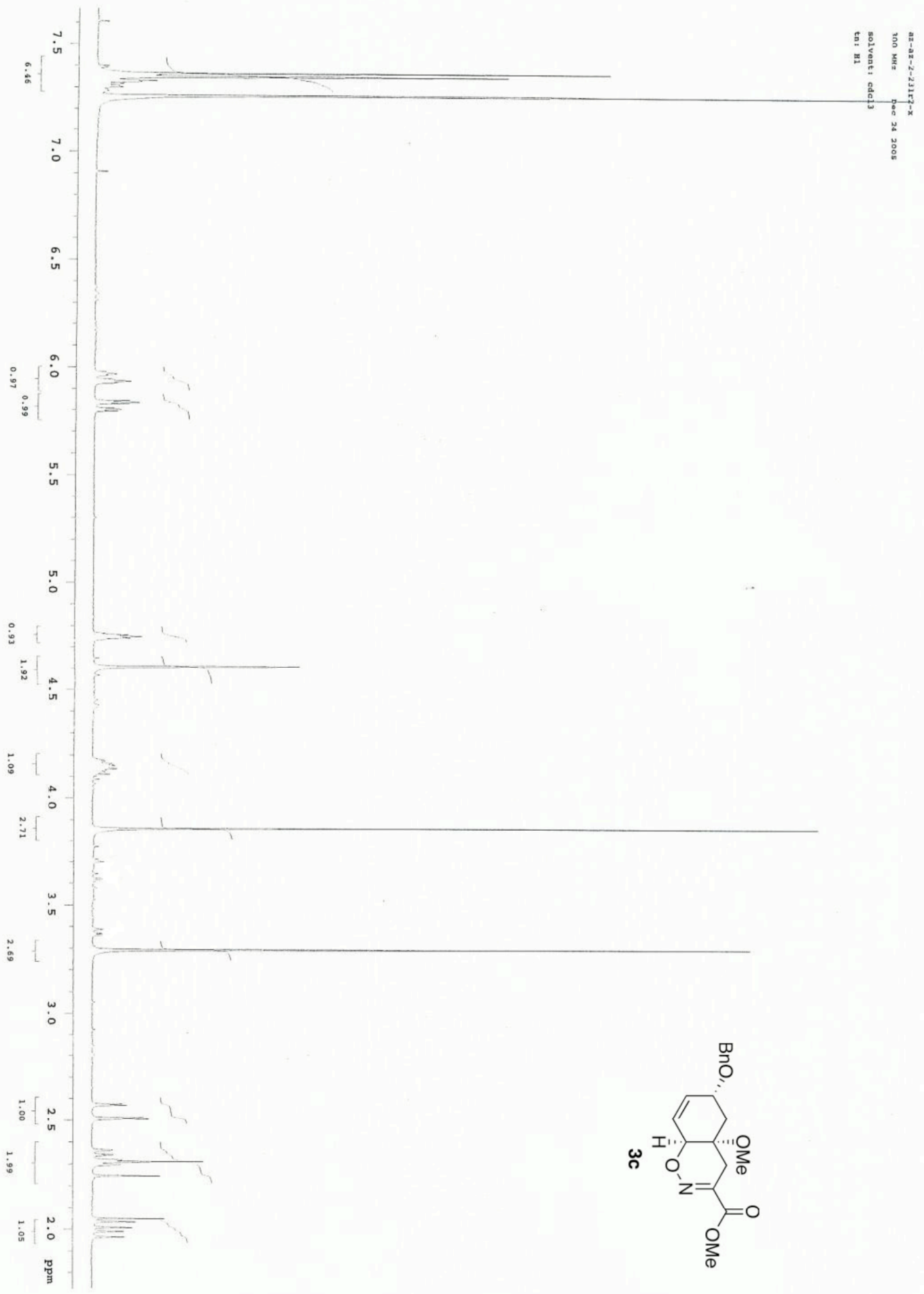




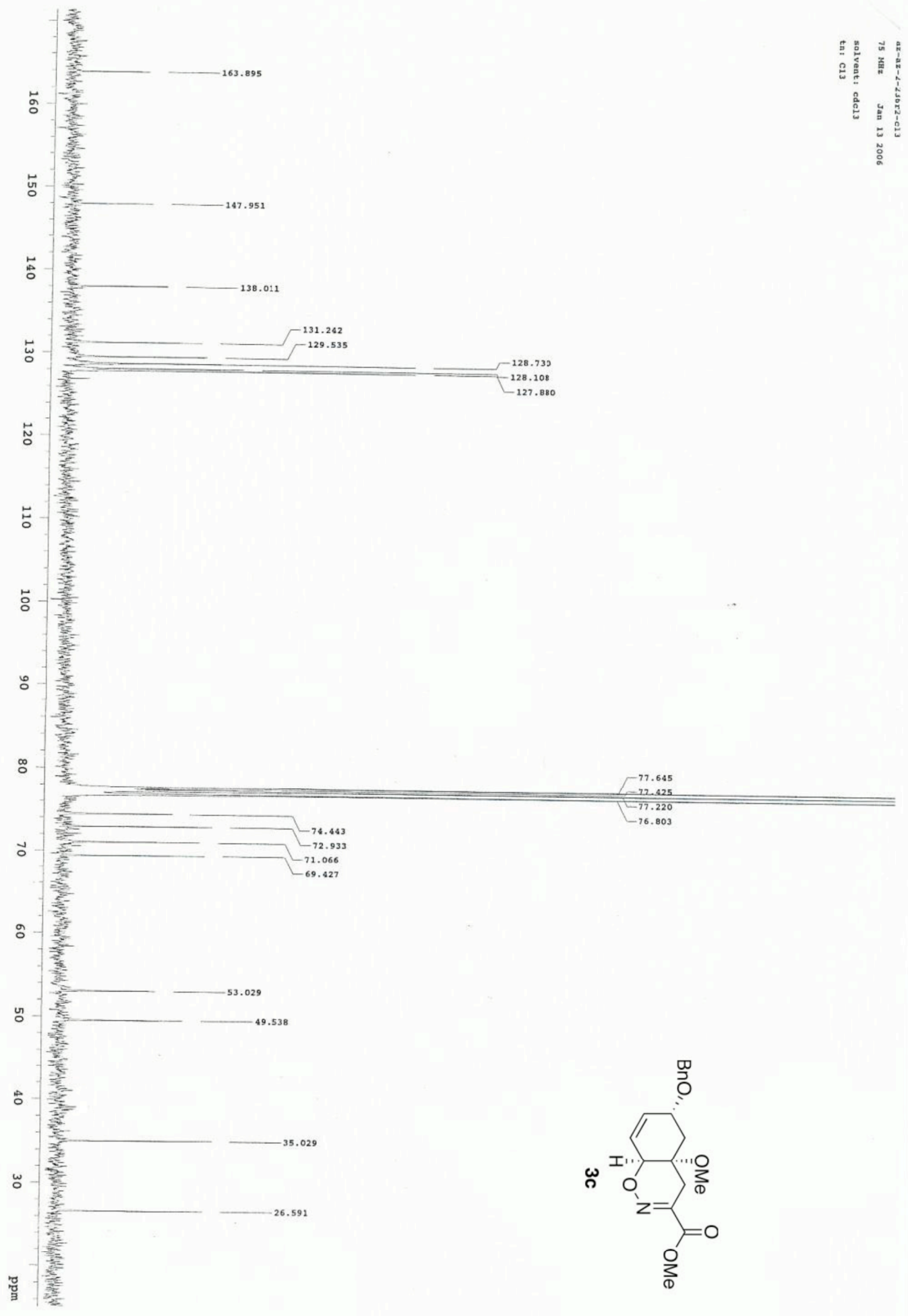




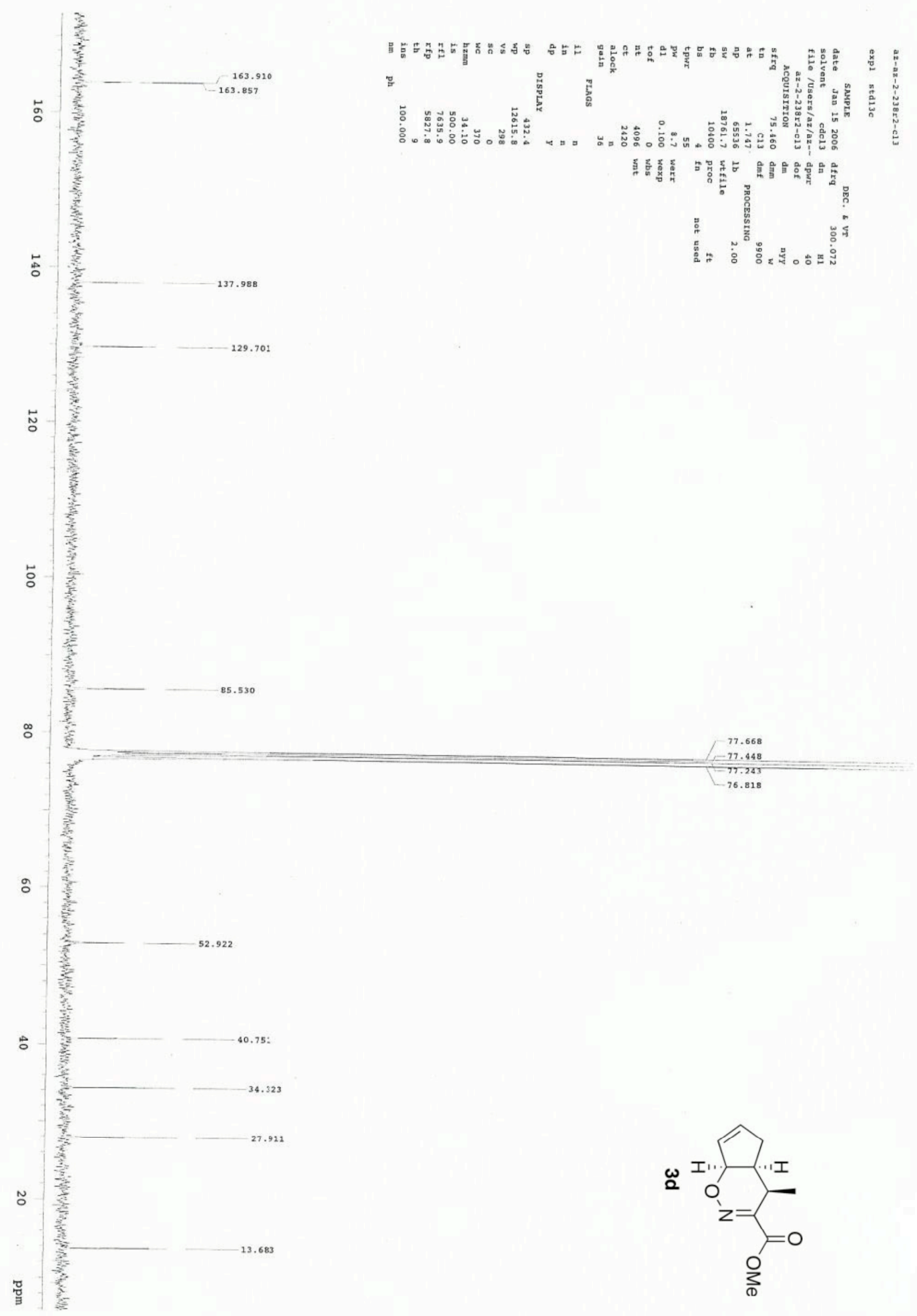




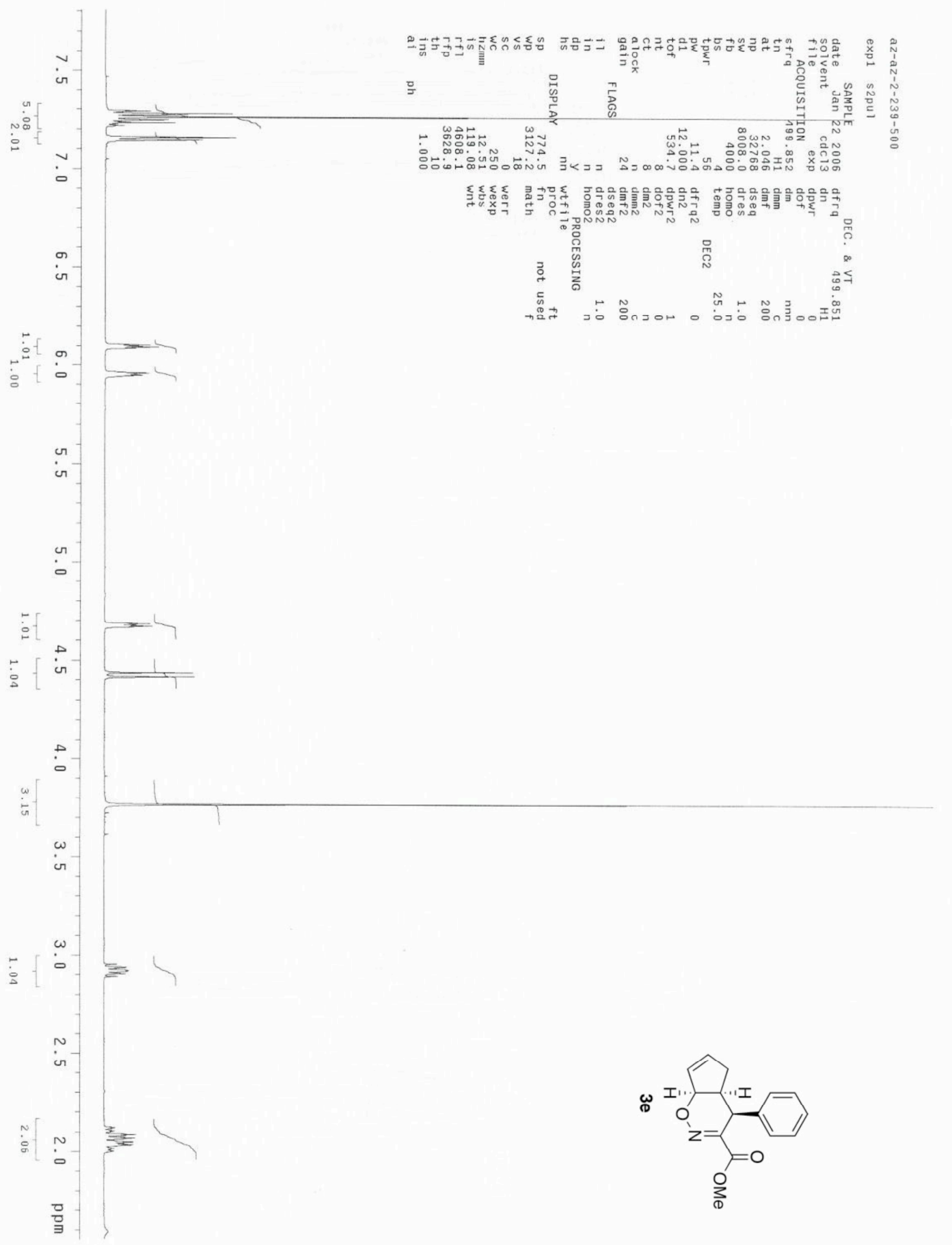




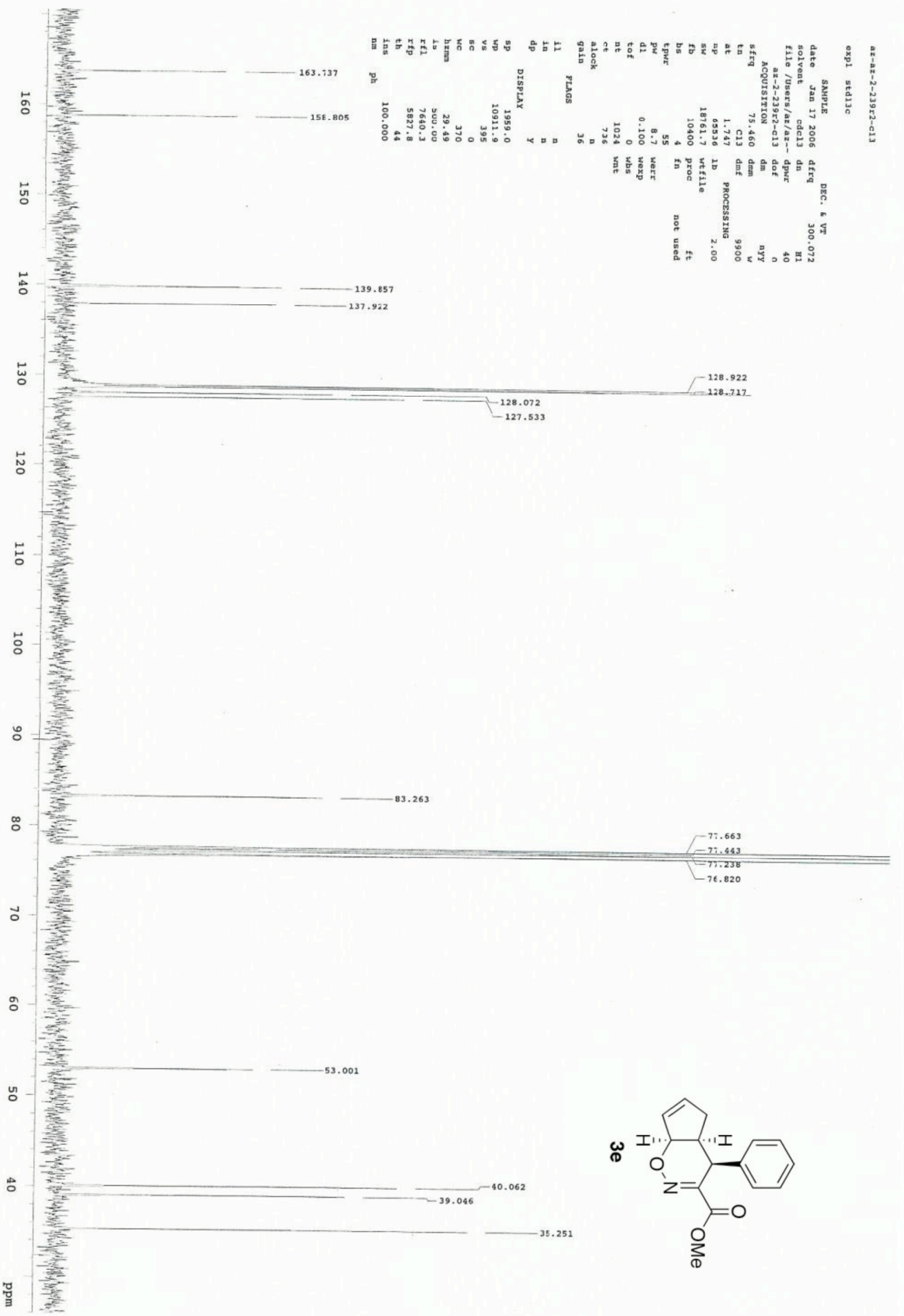




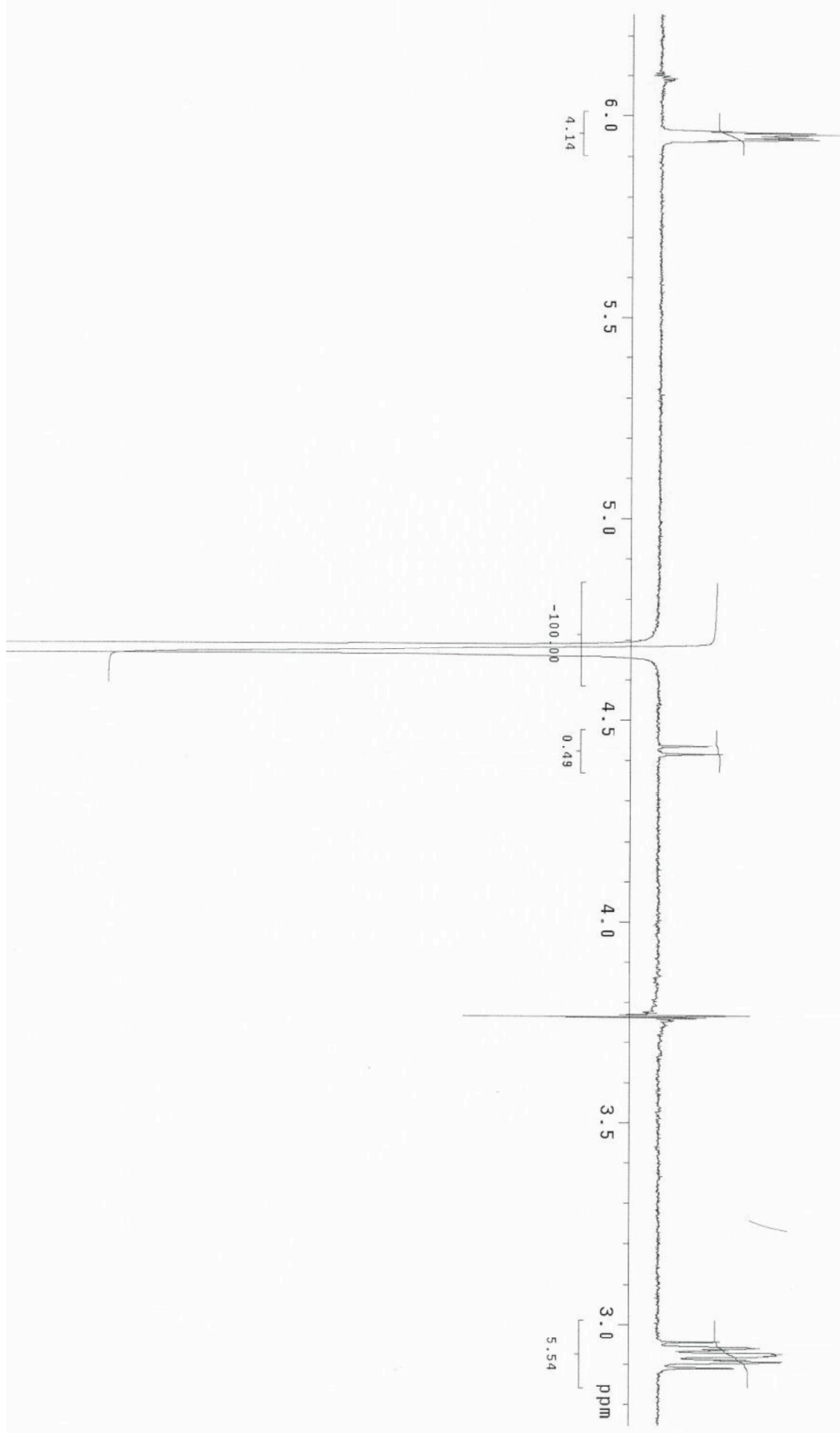




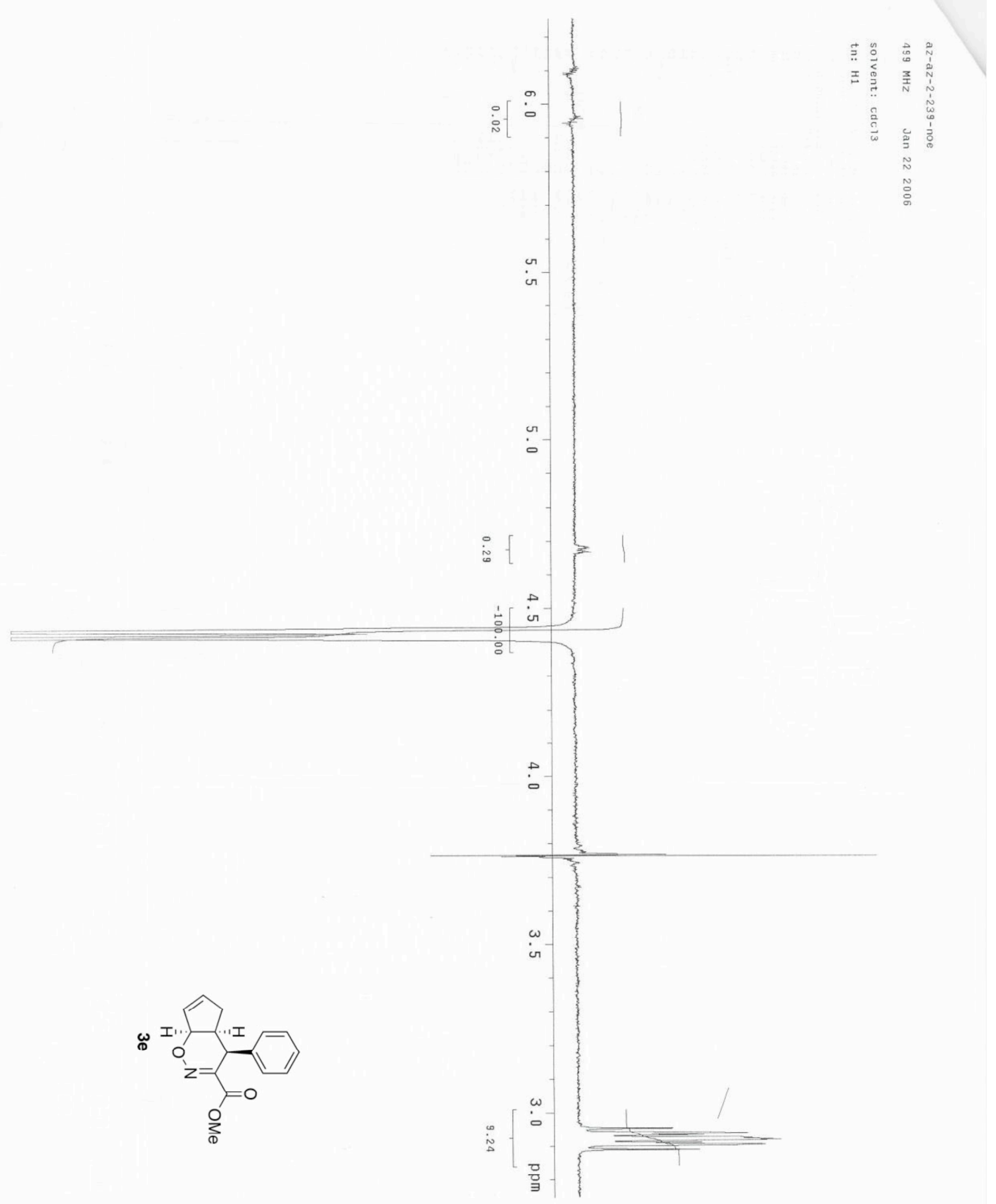




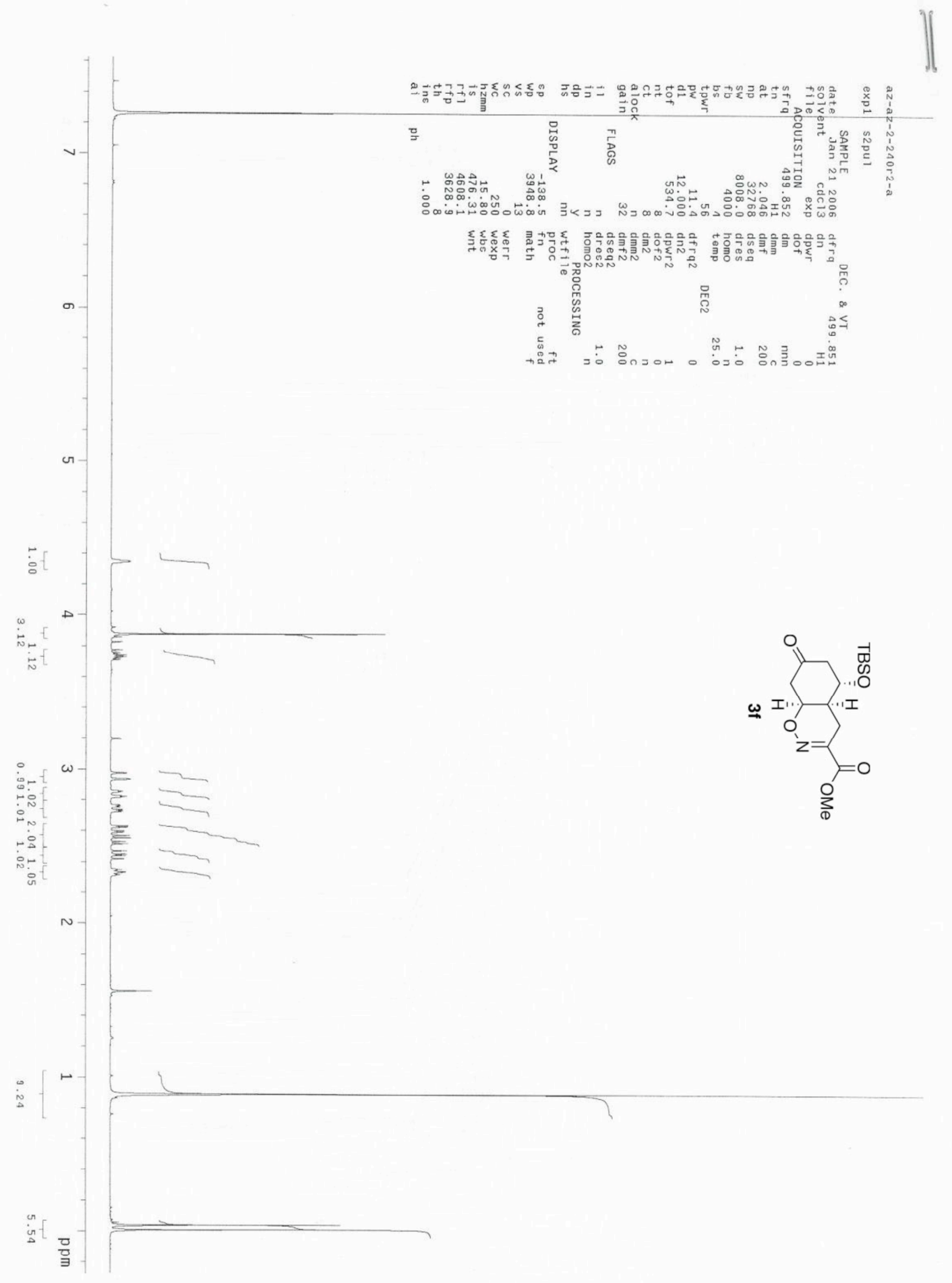




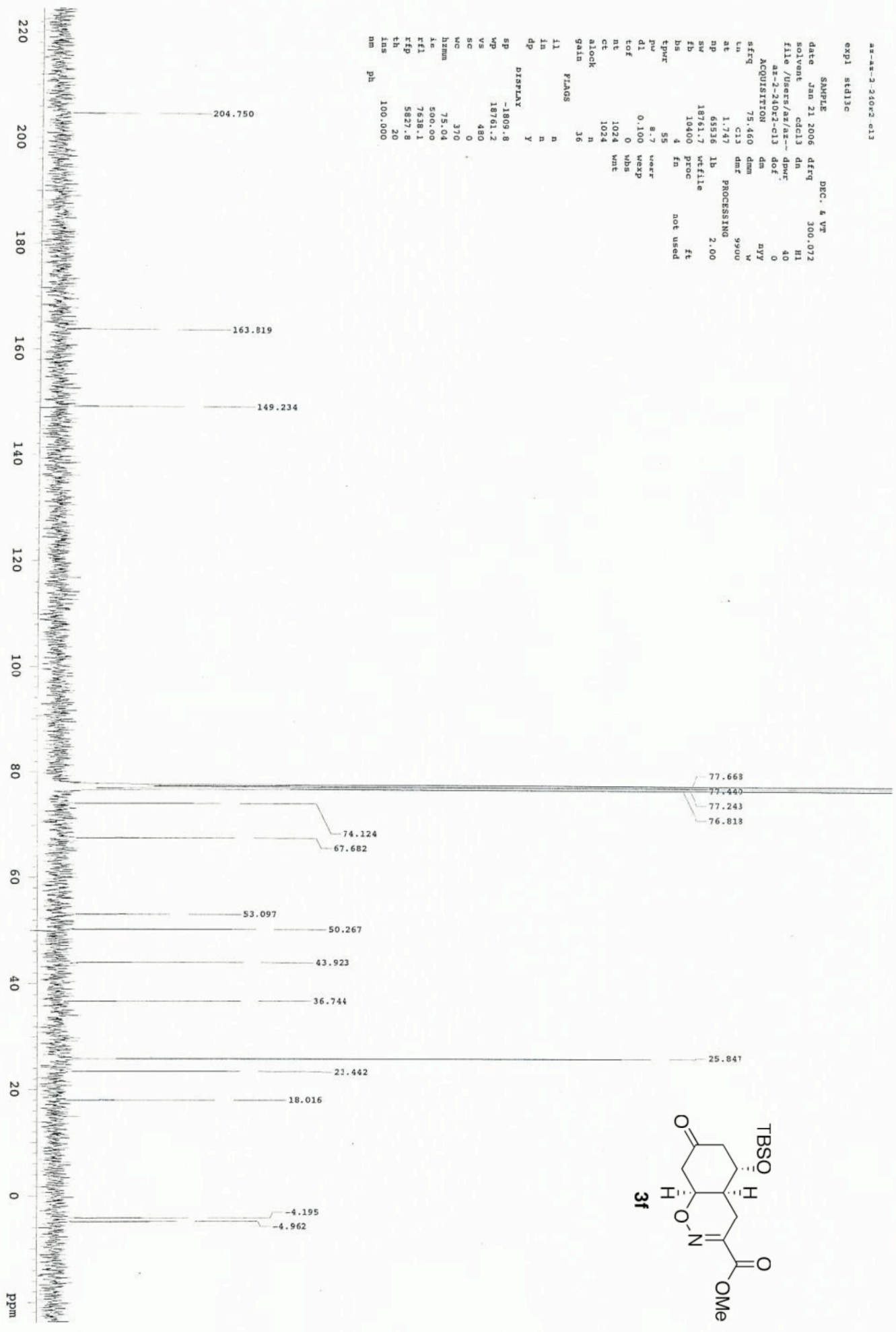



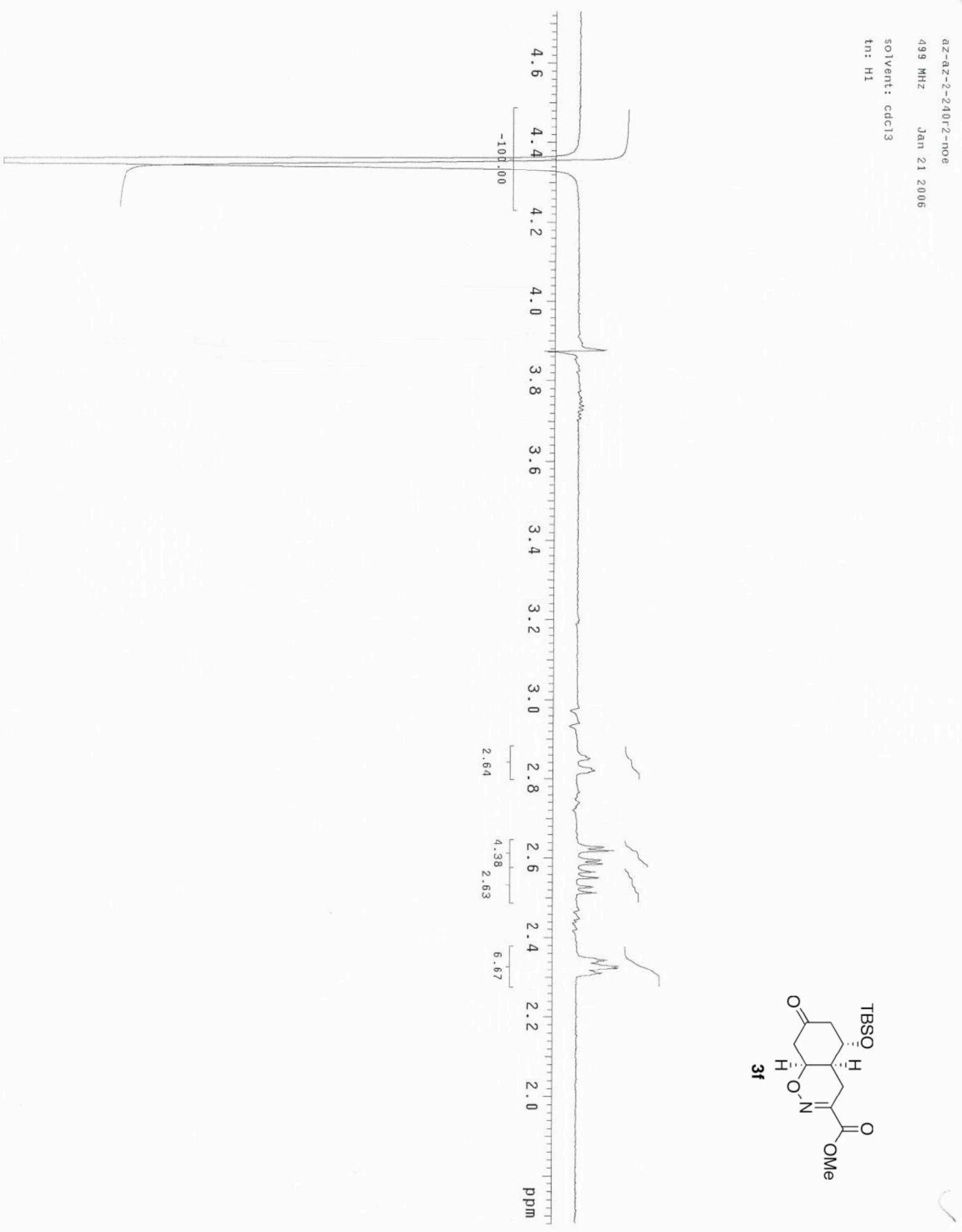

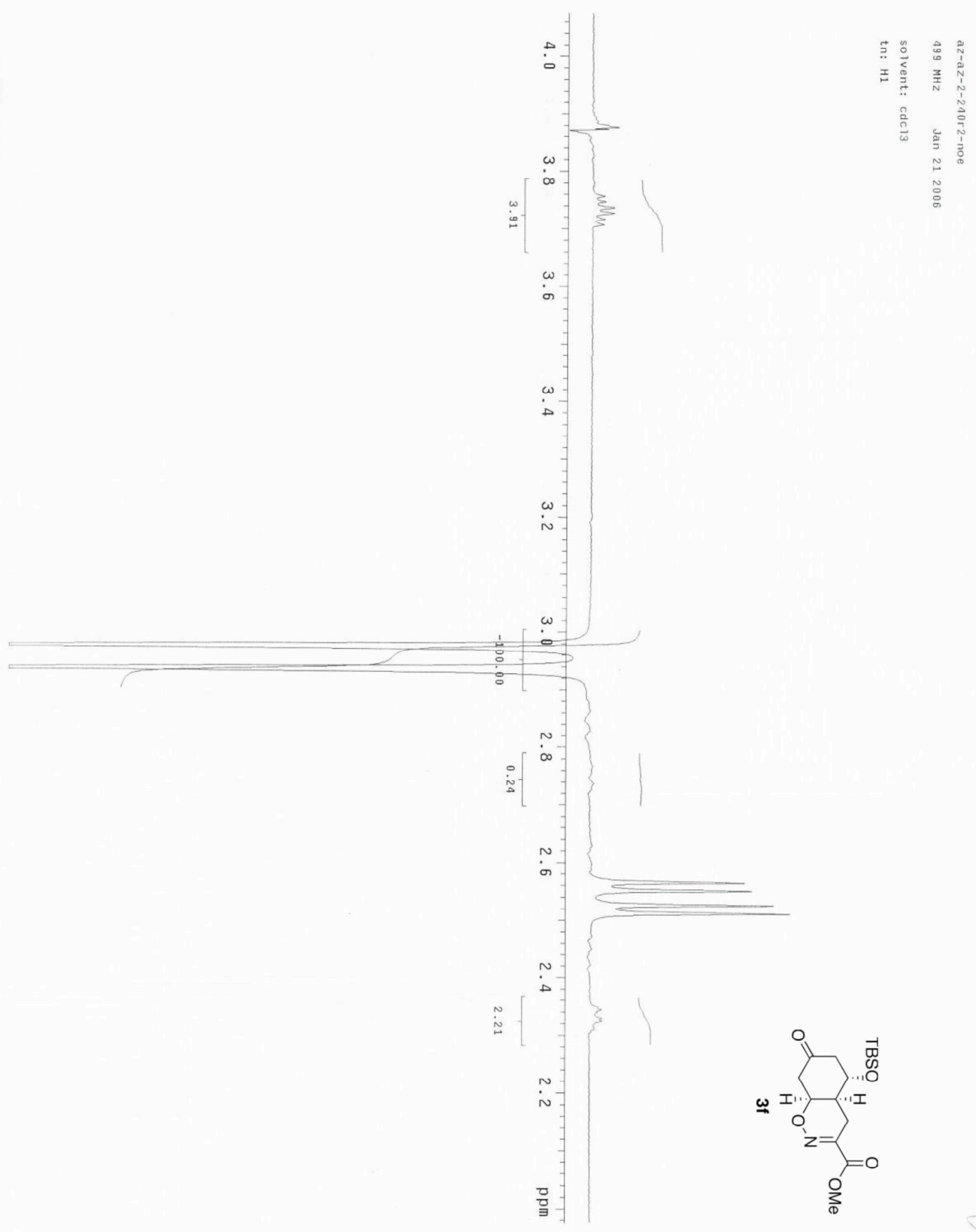


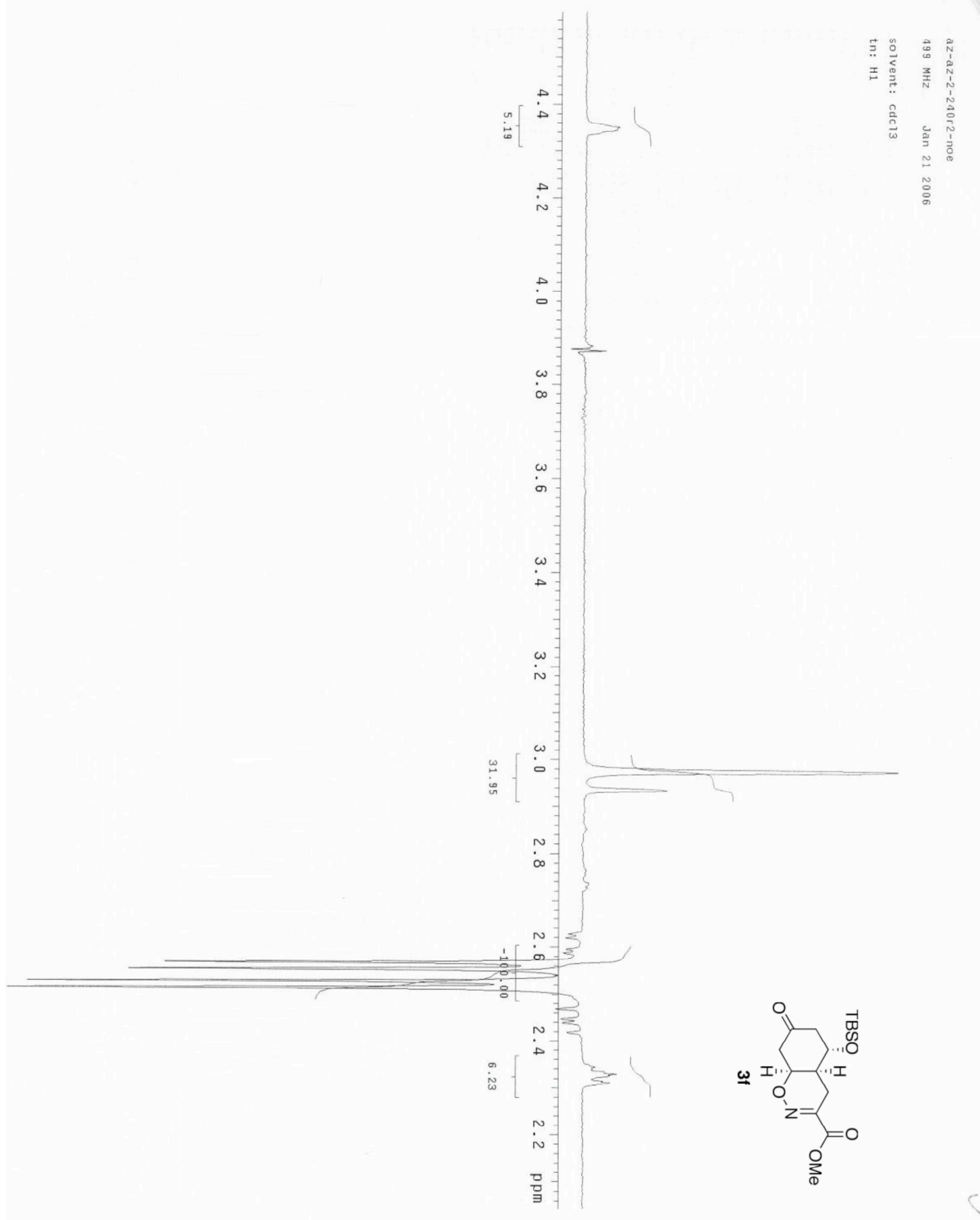




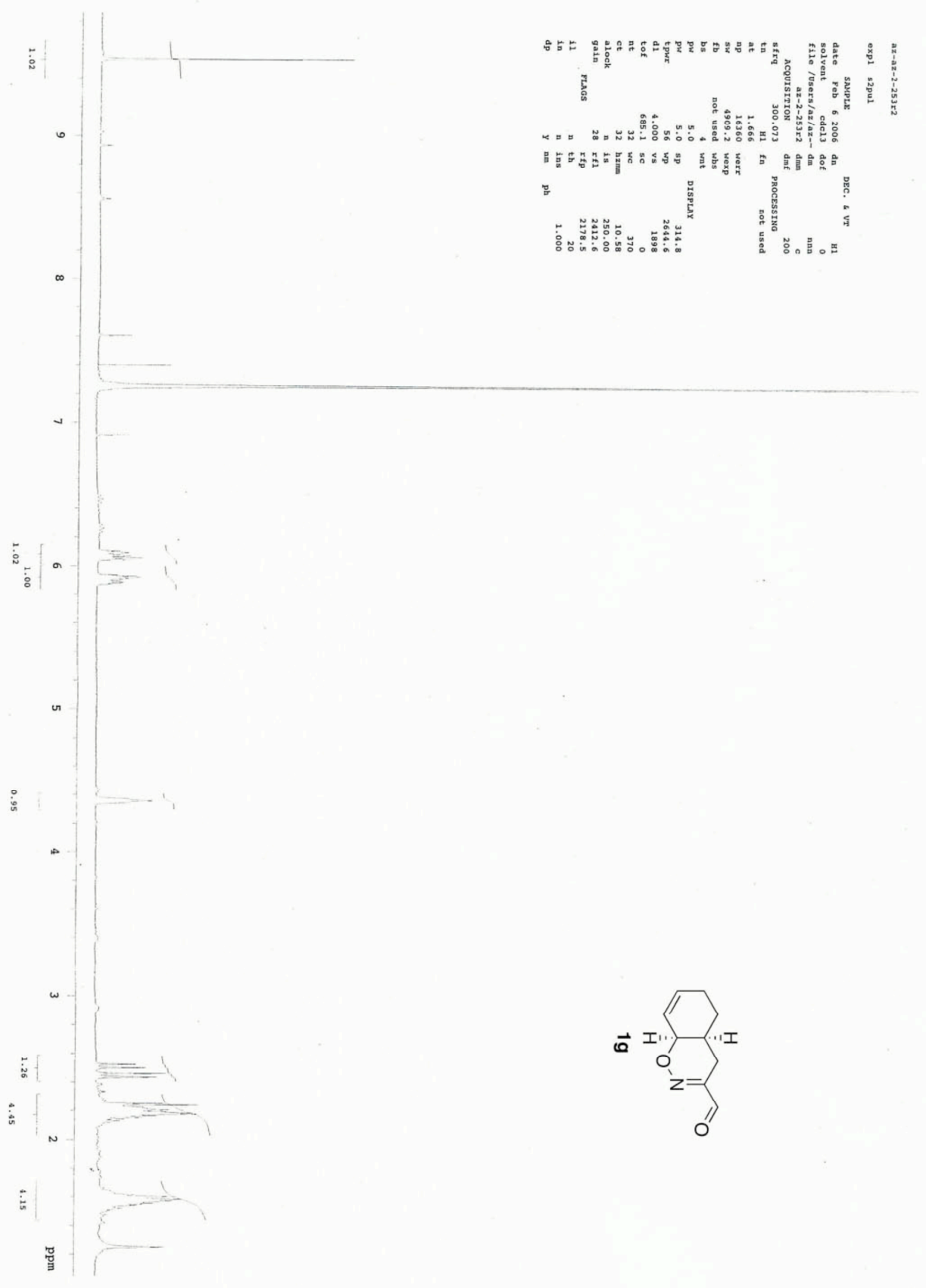




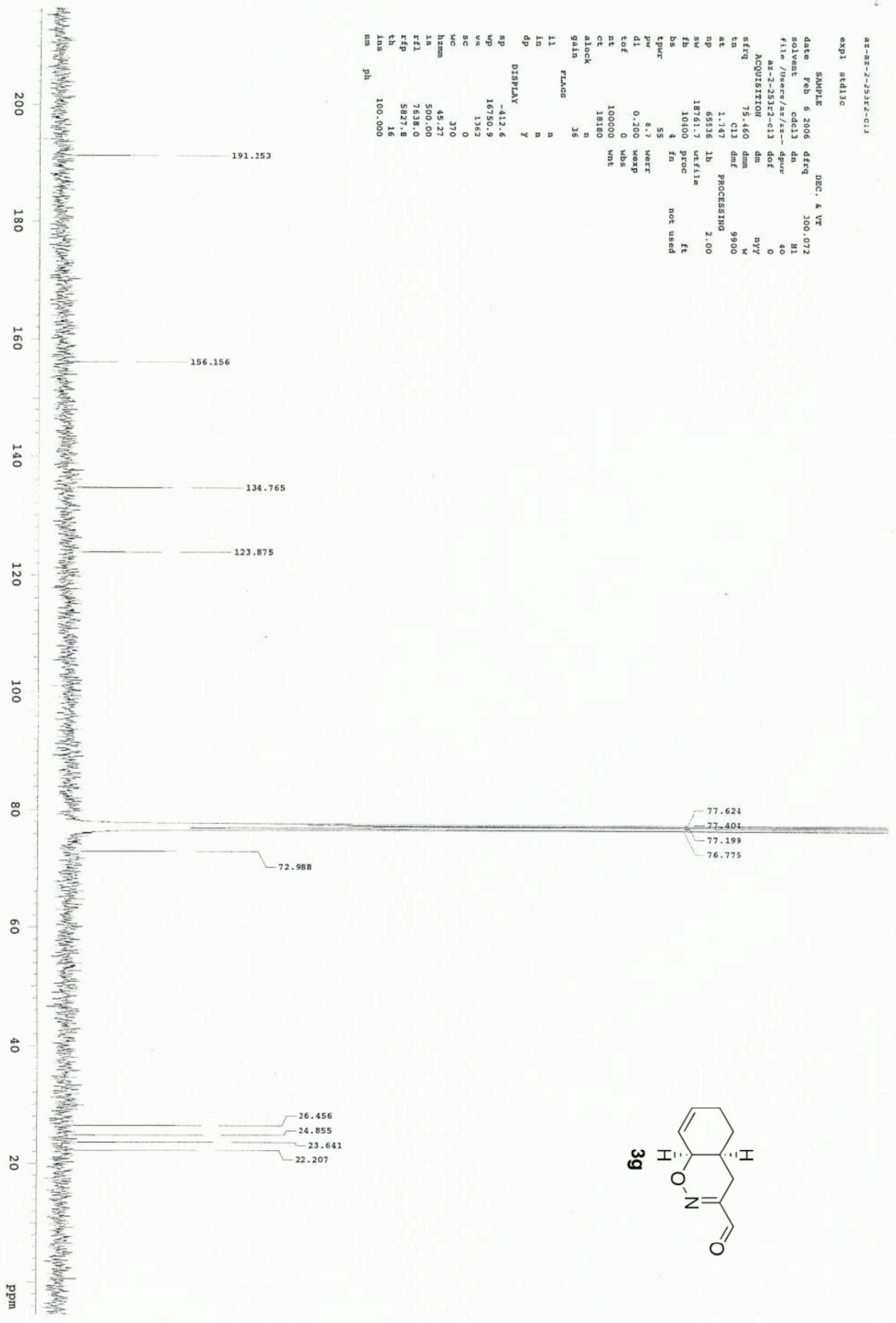

